\title{
On Some Parabolic Type Problems from Thin Film Theory and Chemical Reaction-Diffusion Networks
}

\author{
Fatma Naser Ali Mohamed
}

Follow this and additional works at: https://researchrepository.wvu.edu/etd

\section{Recommended Citation}

Mohamed, Fatma Naser Ali, "On Some Parabolic Type Problems from Thin Film Theory and Chemical Reaction-Diffusion Networks" (2017). Graduate Theses, Dissertations, and Problem Reports. 6241. https://researchrepository.wvu.edu/etd/6241

This Dissertation is protected by copyright and/or related rights. It has been brought to you by the The Research Repository @ WVU with permission from the rights-holder(s). You are free to use this Dissertation in any way that is permitted by the copyright and related rights legislation that applies to your use. For other uses you must obtain permission from the rights-holder(s) directly, unless additional rights are indicated by a Creative Commons license in the record and/ or on the work itself. This Dissertation has been accepted for inclusion in WVU Graduate Theses, Dissertations, and Problem Reports collection by an authorized administrator of The Research Repository @ WVU. For more information, please contact researchrepository@mail.wvu.edu. 


\title{
On Some Parabolic Type Problems from Thin Film Theory and Chemical Reaction-Diffusion Networks
}

\author{
Fatma Naser Ali Mohamed \\ Dissertation submitted \\ to the Eberly College of Arts and Sciences \\ at West Virginia University \\ in partial fulfillment of the requirements for the degree of \\ Doctor of Philosophy in \\ Mathematics
}
Adrian Tudorascu, Ph.D., Chair
Casian Pantea, Ph.D., co-Chair
Harvey Diamond, Ph.D.
Harumi Hattori, Ph.D.,
Tudor Stanescu, Ph.D.
Charis Tsikkou, Ph.D.
Department Of Mathematics
Morgantown, West Virginia
2017

Keywords: Thin-film equations, Asymptotic decay, Energy-energy dissipation, chemical reaction-diffusion systems, method of lines, convergence of method of lines.

Copyright (C) 2017 Fatma Naser Ali Mohamed 


\title{
ABSTRACT \\ On Some Parabolic Type Problems from Thin Film Theory and Chemical Reaction-Diffusion Network
}

\author{
Fatma Naser Ali Mohamed
}

This dissertation considers some parabolic type problems from thin film theory and chemical reaction-diffusion networks. The dissertation consists of two parts:

In the first part we study the evolution of a thin film of fluid modeled by the lubrication approximation for thin viscous films. We prove existence of (dissipative) strong solutions for the Cauchy problem when the sub-diffusive exponent ranges between $3 / 8$ and 2 ; then we show that these solutions tend to zero at rates matching the decay of the source-type self-similar solutions with zero contact angle. We introduce the weaker concept of dissipative mild solutions and we show that in this case the surface-tension energy dissipation is the mechanism responsible for the $H^{1}$-norm decay to zero of the thickness of the film at an explicit rate. Relaxed problems, with second-order nonlinear terms of porous media type are also successfully treated by the same means.

In the second part we are concerned with the convergence of a certain space-discretization scheme -the so-called method of lines- for mass-action reaction-diffusion systems. First, we start with a toy model, namely

$$
A \underset{k_{1}}{\stackrel{k_{-1}}{\leftrightarrows}} B
$$

and prove convergence of method of lines for this linear case. Here weak convergence in $L^{2}(0,1)$ is enough to prove convergence of the method of lines. Then we adopt the framework for convergence analysis introduced in [23], and concentrate on the proof-of-concept reaction

$$
A+B \underset{k_{1}}{\stackrel{k_{-1}}{\leftrightarrows}} C
$$

within 1D space, while at the same time noting that our techniques are readily generalizable to other reaction-diffusion networks and to more than one space dimension. Indeed, it will be obvious how to extend our proofs to the multi-dimensional case; we only note that the proof of the comparison 
principle (the continuous and the discrete versions; see chapter 6) imposes a limitation on the spatial dimension (should be at most five; see [24] for details). The Method of Lines (MOL) is not a mainstream numerical tool and the specialized literature is rather scarce. The method amounts to discretizing evolutionary PDE's in space only, so it produces a semi-discrete numerical scheme which consists of a system of ODE's (in the time variable). To prove convergence of the semi-discrete MOL scheme to the original PDE one needs to perform some more or less traditional analysis: it is necessary to show that the scheme is consistent with the continuous problem, and that the discretized version of the spatial differential operator retains sufficient dissipative properties in order to allow an application of Gronwall's Lemma to the error term. As shown in [23], a uniform (in time) consistency estimate is sufficient to obtain convergence; however, the consistency estimate we proved is not uniform for small time, so we cannot directly employ the results in [23] to prove convergence in our case. Instead, we prove all the required estimates "from the scratch", then we use their exact quantitative form in order to conclude convergence. 


\section{DEDICATION}

I would like to dedicate this work to

My parents, my husband, my children, my siblings. 


\section{ACKNOWLEDGEMENTS}

First and foremost, I am most indebted to my advisor Dr. Adrian Tudorascu and my co-advisor Dr. Casian Pantea for their continued encouragement and support over these last few years. It has been a pleasure to work under their supervision. Without them, this dissertation could not have come about. Also, I would like to thank Dr.Pantea for providing me with financial support as a research assistant: Part I is joint work with Dr. Adrian Tudorascu, and Part II is joint work with Dr. Casian Pantea and Dr. Adrian Tudorascu.

I would also like to thank the other members of my graduate committee, Dr. Harvey Diamond, Dr. Harumi Hattori, Dr. Tudor Stanescu and Dr.Charis Tsikkou for their contributions to this thesis and for serving on this committee.

And finally, I would like to take this opportunity to extend great thanks to the Department of Mathematics and Eberly College of Arts and Sciences at West Virginia University for providing me with an excellent study environment and financial support during my study as a graduate student, teaching assistant and research assistant. 


\section{Contents}

Acknowledgments $\quad$ v

List of Figures $\quad$ viii

$\begin{array}{ll}\text { Preface } & 1\end{array}$

I Thin viscous films: thinning driven by surface-tension energy $\begin{array}{ll}\text { dissipation } & 6\end{array}$

1 Preliminaries $\quad 7$

1.1 Theoretical Background . . . . . . . . . . . . . . . . . . 7

1.2 Derivation of the Thin Film Equation . . . . . . . . . . . . . . . . . 9 9

1.3 Degenerate diffusion equations: fourth order versus second order . . . . . . 11

1.4 A definition of entropy . . . . . . . . . . . . . . . . . . . 12

2 Existence and regularity results $\quad 14$

2.1 Existence results for the thin film equations in a bounded domain . . . . . . 14

2.2 Existence results for the Cauchy problem . . . . . . . . . . . . . . 24

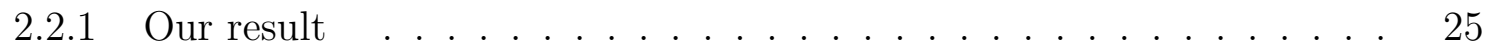

2.3 Existence result for a relaxed problem . . . . . . . . . . . . . . . 31

3 Asymptotic decay $\quad 34$

3.1 Asymptotic decay for a thin film equation in a bounded domain . . . . . . . 34

3.2 Asymptotic decay for the Cauchy problem . . . . . . . . . . . . . . . 35

3.2.1 Our result for the Cauchy problem . . . . . . . . . . . . 35

3.3 Our result for the relaxed problem . . . . . . . . . . . . . . . . . . 41

II Chemical reaction-diffusion networks; convergence of the method of lines $\quad 45$

4 Preliminaries $\quad 46$

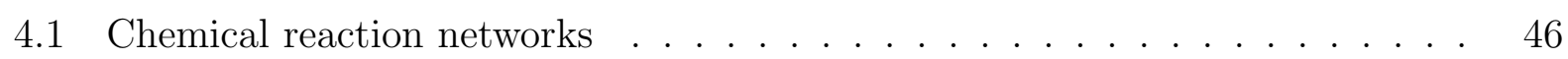

4.2 Well-mixed chemical reaction networks . . . . . . . . . . . . . . 47

4.2 .1 Law of mass action . . . . . . . . . . . . . . . . . . . . 47 
4.3 Chemical reaction-diffusion networks $(\mathrm{CRDN}) \ldots \ldots \ldots$

4.4 The method of lines (Semi-discretizations) . . . . . . . . . . . . . 50

4.4.1 Method of lines for chemical reaction-diffusion networks . . . . . . . . 51

4.4.2 The connection between MOL and CRDN . . . . . . . . . . . . 52

5 Convergence of the Method of Lines: linear case 58

6 Convergence of the Method of Lines: nonlinear case $\quad 67$

6.0.1 Discretization by the Method of Lines (MOL) . . . . . . . . . . 68

6.1 The Comparison Principle (Continuous and semi-discrete Problems) . . . . . 71

6.2 Convergence . . . . . . . . . . . . . . . . . . . 77

6.2.1 A consistency estimate . . . . . . . . . . . . . . 79

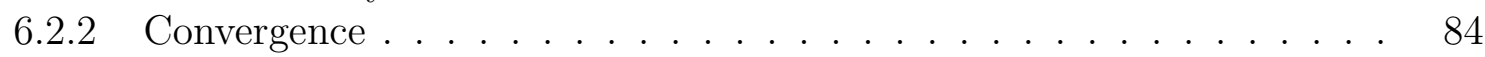

7 Appendix $\quad 92$

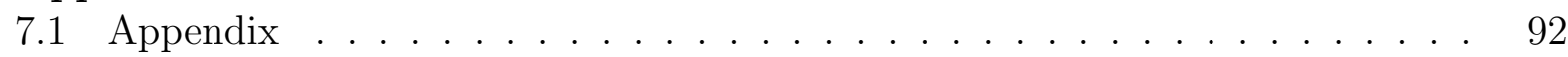

7.1.1 Dirichlet Heat Kernel/Neumann Heat Kernel . . . . . . . . . . . . . . 92

7.1.2 The Neumann Heat Kernel associated to the discrete case . . . . . . 96

7.1.3 Convergence of $H^{N}(t, x, y)$ to $H(t, x, y) \ldots \ldots \ldots$. . . . . . 97

7.1 .4 A special function . . . . . . . . . . . . . . . . . 99

7.1.5 Convergence of $a_{0}^{N}(x)$ to $a_{0}$ in $L^{p}(0,1) \ldots \ldots \ldots$. . . . . . 101

$\begin{array}{ll}\text { Bibliography } & 103\end{array}$ 


\section{List of Figures}

1.1 A: non-wetting, B: high wetting, C: low wetting and S: perfect wetting.(see

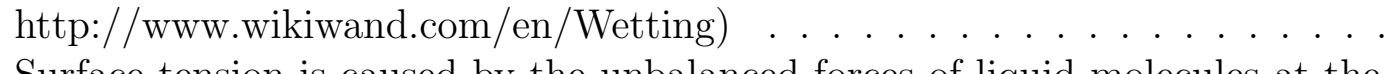

1.2 Surface tension is caused by the unbalanced forces of liquid molecules at the surface (see https://chem.libretexts.org) . . . . . . . . . . . 9 9

1.3 Illustration of contact angles formed by a liquid drop on a smooth homogeneous solid surface . . . . . . . . . . . . . . . . . . . . 9

4.1 Boxes $k-1, k$ and $k+1$; linear case . . . . . . . . . . . . . 54

6.1 Boxes $k-1, k$ and $k+1$ : nonlinear case . . . . . . . . . . . 69 


\section{Preface}

This dissertation focuses on some parabolic type problems from thin film theory (part I) and chemical reaction-diffusion networks (part II).

The equation analyzed in the first part is

$$
\left\{\begin{array}{cl}
\partial_{t} u(t, x)=-\partial_{x}\left(u^{n}(t, x) \partial_{x}^{3} u(t, x)\right) & \text { in } Q:=(0, \infty) \times \mathbb{R} \\
u(0, \cdot)=u_{0}(x) & \text { in } \mathbb{R} .
\end{array}\right.
$$

Equation (2.38) is derived from a lubrication approximation and models the surface-tension dominated motion of thin viscous films and spreading droplets [36]. The case $n=1$ models the flow in a Hele-Shaw cell; $n=3$ models the viscous flow on a solid surface without slip driven by surface tension.

The goal pursued in this work is two-fold. First, we prove existence of dissipative strong solutions for the Cauchy problem, thus extending the result by Carrillo and Toscani [13] $(n=1)$ to $3 / 8<n<2$. As in [13], these solutions arise as limits of the compactly supported strong solutions found in [6]. Consequently, they will satisfy the same rates of decay as the ones proved in [6] to be optimal (they match the decay rates of the self-similar solutions with zero contact angle). Secondly, we consider dissipative weak solutions with slightly higher regularity, which we call dissipative mild solutions, for the Cauchy problem and show that they asymptotically converge to zero at an explicit rate. The point we make is that the surface-tension energy dissipation is the mechanism responsible for the asymptotic decay. The model itself is derived from the Navier-Stokes equation when the typical thickness $u$ of the film is much smaller than the typical length of the region occupied by the fluid [43]. Furthermore, nonnegative solutions $u$ satisfying, for some $t_{0} \geq 0, u\left(t_{0}, \cdot\right) \in H^{2}(\mathbb{R})$ have the obvious property that $u_{x}\left(t_{0}, x_{0}\right)=0$ whenever $u\left(t_{0}, x_{0}\right)=0$. This is known as the zero contact angle property. It is expected for a physically correct model that the thickness of the film decays to zero as $t \rightarrow \infty$. Therefore, we argue that physical solutions should be dissipative, i.e. they should satisfy an inequality of the form (2.45) below.

The existence of nonnegative weak solutions for this problem in a bounded domain was proved by Bernis and Friedman [7] for $n \geq 1$, and Bertozzi and Pugh [10] for all $n>0$. In [6], the author shows that strong solutions for the natural boundary-value problem (first and third derivatives are zero at the boundary) can be used to construct a strong solution for the Cauchy problem. These solutions are obtained as accumulation points in the appropriate 
topology of classical solutions for regularized problems of the type

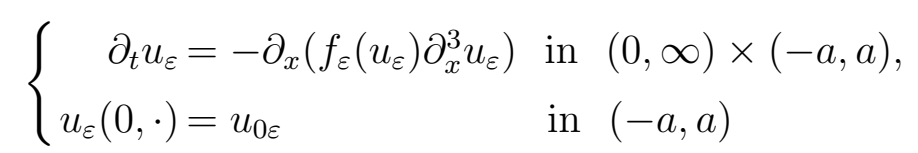

with boundary conditions

$$
\partial_{x} u_{\varepsilon}(t, \pm a)=0=\partial_{x}^{3} u_{\varepsilon}(t, \pm a) \quad \text { for } t>0
$$

where $f_{\varepsilon}(s)$ is an appropriate one-parameter modification of the function $s^{n}$ with the purpose of avoiding parabolic degeneracy [7]. Uniqueness of these strong solutions is not known. Thus, the results in [6] and [13] are proved for the strong solutions arising as limits of these standard regularized problems. The rates of uniform decay to zero obtained in [6] for $0<n<2$ are shown to be optimal, in the sense that they match the rates of decay for the corresponding self-similar solutions [8]

$$
h(t, x)=t^{-\alpha} H\left(t^{-\alpha} x\right), \quad \alpha=\frac{1}{n+4},
$$

where $H$ is even, compactly supported, solves $H^{n}(x) H^{\prime \prime \prime}(x)=\alpha x H(x)$ and satisfies $H^{\prime}=0$ at the edge of the support.

If the first equation is formally differentiated with respect to $x$ and then integrated by parts against $u_{x}$, by assuming sufficiently fast decay for $|x| \rightarrow \infty$ we obtain

$$
\frac{1}{2} \frac{d}{d t} \int_{\mathbb{R}}\left(\partial_{x} u(t, x)\right)^{2} d x=-\int_{\mathbb{R}} u^{n}(t, x)\left(\partial_{x}^{3} u(t, x)\right)^{2} d x
$$

Thus, the surface-tension energy

$$
J[u]:=\frac{1}{2} \int_{\mathbb{R}}\left(\partial_{x} u(t, x)\right)^{2} d x
$$

is a Lyapunov functional for nonnegative smooth solutions of (2.38). If we formally integrate (2.38) over $I$ for any fixed $t>0$, we observe that the total mass of $u(t, \cdot)$ is conserved in time. However, smooth solutions are, in general, not known to exist. Thus, only a statement 
weaker than (1.5) does hold for the strong solutions constructed in [6], namely

$$
J[u(t, \cdot)]+\int_{0}^{t} \int_{\mathcal{P}_{\tau}} u^{n}(\tau, x)\left(\partial_{x}^{3} u(\tau, x)\right)^{2} d x d \tau \leq J\left[u_{0}\right],
$$

where $\mathcal{P}_{t}:=\{x \in \mathbb{R}: u(t, x)>0\}$. This comes as the limit as $\varepsilon \downarrow 0$ of the identity replacing (0.6) in the case of $u_{\varepsilon}$, i.e.

$$
J\left[u_{\varepsilon}(t, \cdot)\right]+\int_{0}^{t} \int_{\mathbb{R}} f_{\varepsilon}\left(u_{\varepsilon}(\tau, x)\right)\left(\partial_{x}^{3} u_{\varepsilon}(\tau, x)\right)^{2} d x d \tau=J\left[u_{0 \varepsilon}\right] .
$$

The limiting strong solution $u$ also satisfies, for $0<n<2$, the entropy dissipation

$$
\frac{d}{d t} \int_{\mathbb{R}} u^{3-n}(t, x)=-(2-n)(3-n) \int_{\mathbb{R}} u(t, x)\left(\partial_{x}^{2} u(t, x)\right)^{2} d x \quad \text { for a.e. } t>0 .
$$

This inequality is instrumental to obtaining the optimal decay rates in [6]. To that end, it is used in conjunction with (0.7).

Our main goal for the first part is to show that a surface-tension energy dissipation inequality (satisfied by the strong solutions presented below) is enough for proving the asymptotic decay to zero of $J[u(t, \cdot)]$ under much weaker regularity assumptions on $u$ than in [6]. This approach is elementary and natural and yields one rate of decay for all $0<n<2$.

The structure of this first part is the following: in chapter 2 we generalize the existence result from [13] to the case $3 / 8<n<2$. In order to accommodate the range $3 / 8<n<1$ we introduce a weaker notion of solutions than the one in [13]. In chapter 3 we prove the decay to zero in the $H^{1}$-norm for dissipative mild solutions: in particular, this holds for the strong solutions constructed in [6]. In fact, our methods work for the case $0<n<2$. Even though for $0<n \leq 3 / 8$ we have no proof of existence of weak solutions originating from noncompactly supported initial data, our result applies to solutions stemming from compactly supported data, which are constructed in [6]. Then we quantify the effect of "porous media" type perturbations on the decay rates obtained for the original problem (2.38). We show how the analysis performed in the previous chapters works here as well. In fact, the second-order perturbation allows for better decay rates.

The second part of this dissertation concerns chemical reaction-diffusion systems. In systems biology, the dynamical behavior of spatially homogeneous mass-action reaction systems has been the focus of much recent research. A great number of results on the possibility of 
bistability or oscillation, local and global stability of equilibria, persistence of solutions etc. have been developed for ODE systems corresponding to well-mixed reaction networks. This effort started forty years ago [30], [29], [25], and has seen a surge of interest in more recent years: [40], [41], [20], [21], [3], [34], [2], [16], [19], [1], [22], [4], to cite but a few examples. In particular, some of this work led to a proof of the Global Attractor Conjecture [18], a global asymptotic stability result for a large class of systems (called complex balanced networks).

On the other hand, much less is known about the corresponding reaction-diffusion setting, where most of the effort has focused on the asymptotic behavior of solutions. One of the most studied examples is the reaction-diffusion system $A+B \leftrightharpoons C$, whose solutions approach a spatially homogeneous distribution; this was shown by way of semigroup theory [37] and entropy methods [23]. The entropy method has been used to successfully tackle other reaction-diffusion systems, including dimerization systems $2 A \leftrightharpoons B$ [23], weakly reversible monomolecular reactions and other classes of linear systems [26], and classes of complex balanced systems without boundary equilibria [24]. The latter work lays out a general method for complex balanced systems, but some of the technicalities depend on the specific network considered. This difficulty goes away under the assumption of equal diffusion coefficients, where general results on the asymptotic stability of positive equilibria have been shown in $[35]$.

In this work our focus is different than that of the literature cited above. Namely, we are concerned with the convergence of a certain space-discretization scheme - the so-called method of lines- for mass-action reaction-diffusion systems. We adopt the framework for convergence analysis introduced in [44], and concentrate on the proof-of-concept reaction

$$
A+B \underset{k_{1}}{\stackrel{k_{-1}}{\leftrightharpoons}} C
$$

within 1D space, while at the same time noting that our techniques are readily generalizable to other reaction-diffusion networks and to more than one space dimension. Indeed, it will be obvious how to extend our proofs to the multi-dimensional case; we only note that the proof of the comparison principle (the continuous and the discrete versions; see 6.1) imposes a limitation on the spatial dimension (should be at most five; see [14] for details).

The Method of Lines (MOL) is not a mainstream numerical tool and the specialized literature is rather scarce. The method amounts to discretizing evolutionary PDE's in space only, so it produces a semi-discrete numerical scheme which consists of a system of ODE's 
(in the time variable). To prove convergence of the semi-discrete MOL scheme to the original PDE one needs to perform some more or less traditional analysis: it is necessary to show that the scheme is consistent with the continuous problem, and that the discretized version of the spatial differential operator retains sufficient dissipative properties in order to allow an application of Gronwall's Lemma to the error term. As shown in [44], a uniform (in time) consistency estimate is sufficient to obtain convergence; however, the consistency estimate we proved is not uniform for small time, so we cannot directly employ the results in [44] to prove convergence in our case. Instead, we prove all the required estimates "from the scratch", then we use their exact quantitative form in order to conclude convergence.

The second part of this dissertation is organized as follows. The chapter 4 sets up the notation and preliminaries needed to state our main result, Theorem 6.1. In this chapter there is a brief overview of the convergence result for the method of lines following [44]; namely, convergence is implied by consistency and a uniform bound on the logarithmic norm of the Jacobian matrix of the discretized system. In chapter 5 , we prove convergence of method of lines for $A \leftrightharpoons B$ by using weak convergence in $L^{2}(\Omega)$. In chapter 6 , section 6.1 , we discuss comparison principles for solutions of the reaction-diffusion equation corresponding to 6.1 and for its space discretization, which we then use to prove consistency and boundedness of the logarithmic norm. This completes the proof of Theorem 6.1, and it is done in 6.2.1. Finally, a few technical results regarding the heat kernel and needed in the proof of the main theorem are collected in the Appendix. 


\section{Part I}

Thin viscous films: thinning driven by surface-tension energy dissipation 


\section{Chapter 1}

\section{Preliminaries}

\subsection{Theoretical Background}

Thin-film type evolution processes arise in our everyday life, ranging from very simple processes, such as rain drop movement on a window, to complicated industrial processes such as material coating. Appearing in many physical processes used in industry, thin-film dynamics attracted researchers from physics, mathematics and various engineering departments recently. Due to the large number of industrial applications, understanding the evolution of a thin liquid film surface is extremely important. This may lead to better designs for these processes, making our lives easier. These issues are also an interesting source of mathematical problems, see [43].

The analysis of fluid flow in thin layers is known as lubrication theory. In fluid dynamics, lubrication theory describes fluid flow in a geometry in which one dimension is significantly smaller than the others. The Thin Film Fluid Equation is derived from the Navier Stokes Equation assuming fluid motion on a flat substrate. We also consider viscosity, which is an important fluid property, used to describe the fluidity property or the internal resistance of a fluid to motion (see [45]).

The topic of wetting has received great interest from both fundamental and applied points of view. It plays an important role in many industrial processes, such as oil recovery, lubrication, liquid coating, and printing. If we consider a liquid drop resting on a flat, horizontal solid surface, we define the contact angle as the intersection angle at the three- 
phase boundary liquid/solid/air interface (geometrically, it is defined as the angle between the tangent to the liquid-gas interface and the tangent to the solid interface at the contact line of the three phases). If the surface is in complete wetting, then the contact angle will approach zero. Conversely, if wetting is very small, a spherical droplet is formed and the resulting contact angle reaches 180 degrees. Thus, we can say that, the smaller the contact angle, the greater the surface wettability or surface energy.

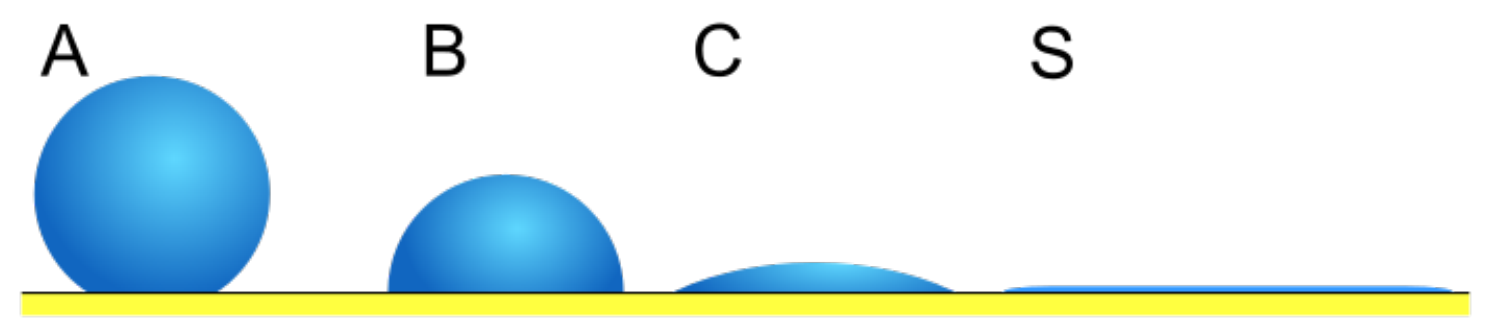

Figure 1.1: A: non-wetting, B: high wetting, C: low wetting and S: perfect wetting.(see http://www.wikiwand.com/en/Wetting)

Wettability studies usually involve the measurement of contact angles as the primary data, which indicate the degree of wetting when a solid and a liquid interact. The surface tension determines the shape of a liquid droplet. In other words, in a pure liquid, each molecule in the bulk pulls its neighboring liquid molecules equally in every direction, which results in zero net force. However, the molecules on the droplet surface do not have neighboring molecules in all directions to provide a balanced net force. Therefore, they are pulled inward by the neighboring molecules, creating an internal pressure. As a result, the liquid naturally contracts its surface area to maintain the lowest surface free energy. This intermolecular force to contract the surface is called the surface tension, and it is responsible for the shape of liquid droplet

As first described by Thomas Young in 1805, the contact angle of a liquid drop on an ideal solid surface is defined by the mechanical equilibrium of the drop under the action of three interfacial tensions:

$$
\sigma_{g s}=\sigma_{l s}+\cos \theta \sigma_{g l},
$$

where $\sigma_{g s}, \sigma_{l s}$, and $\sigma_{g l}$ represent the gas-solid, liquid-solid, gas-liquid and interfacial tensions, respectively, and $\theta$ is the contact angle. (1.1) is usually referred to as Young's equation, and 


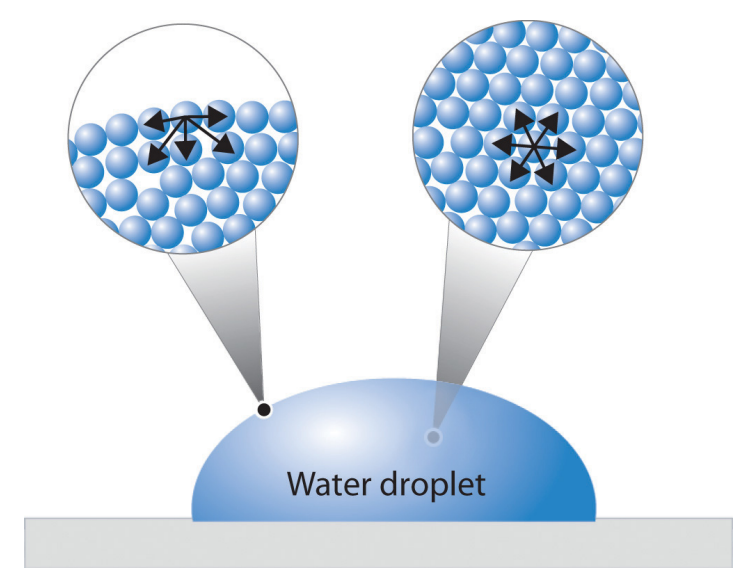

Figure 1.2: Surface tension is caused by the unbalanced forces of liquid molecules at the surface (see https://chem.libretexts.org)

$\theta$ is Young's contact angle.

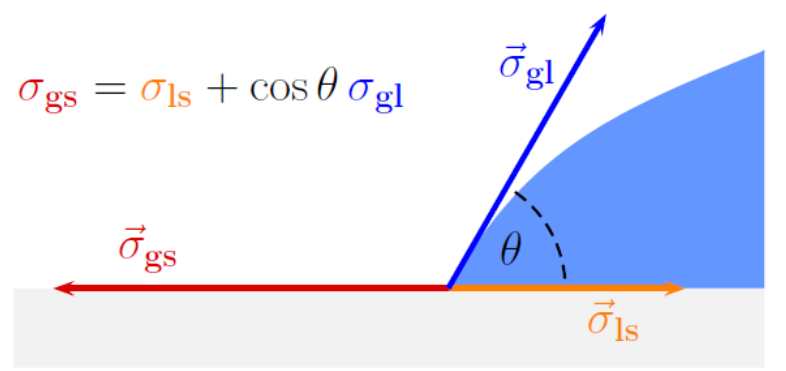

Figure 1.3: Illustration of contact angles formed by a liquid drop on a smooth homogeneous solid surface

In addition, molecules can attract each other at moderate distances and repel each other at close range. The attractive forces are collectively called "van der Waals forces". Van der Waals forces are much weaker than chemical bonds, and random thermal motion around room temperature can usually overcome or disrupt them. Later we will consider the effect of Van der Waals forces that include all intermolecular forces that act between electrically neutral molecules.

\subsection{Derivation of the Thin Film Equation}

$$
\partial_{t} u=-\partial_{x}\left(u^{n} \partial_{x}^{3} u\right)
$$


Let us briefly go through the derivation of this model. We follow [31]. Consider a twodimensional thin flow on a horizontal plate. The flow domain is described by $\{(x, y): 0 \leq$ $y \leq h(t, x)\}$. Assuming the inertia terms to be dominated by the viscosity terms, the NavierStokes equations reduce to

$$
\partial_{x} u+\partial_{y} v=0 ;, \quad-\frac{1}{\rho} \partial_{x} p+\nu \partial_{y}^{2} u=0 ;-\frac{1}{\rho} \partial_{y} p+\nu \partial_{y}^{2} v=g
$$

Here $u$ and $v$ are respectively the velocities in the horizontal $x$-and vertical $y$-direction, $p$ is pressure, $g$ gravity, $\nu$ viscosity and $\rho$ density. The first equation is called the continuity equation since we deal with a Newtonian fluid, the divergence of the velocity field has to be zero. This is equivalent to saying that the volume of the fluid will be conserved.

Assuming that also $\nu \partial_{y}^{2} v$ is neglible one obtains upon integration of the last equation with respect to $y$ that $p=\rho g(h-y)-\partial_{x}^{2} h \sigma$, where $\sigma>0$ reflects surface tension. IF this term is dominant, then the pressure is simply $p=-\partial_{x}^{2} h \sigma$. Going back to (1.3) this tends to

$$
\partial_{y}^{2} u=-\frac{\sigma}{\mu} \partial_{x}^{3} h, \quad \mu=\nu \rho
$$

On the surface of the flow we have a no-stress condition $\partial_{y} u=0$, while on the horizontal plate $u=v=0$ (no-slip condition). Thus

$$
u=\left(y^{2}-2 h y\right) \frac{\sigma}{2 \mu} \partial_{x}^{3} h \Rightarrow \partial_{y} v=-\partial_{x} u=\left(y^{2}-2 h y\right) \frac{\sigma}{2 \mu} \partial_{x}^{4} h-y \frac{\sigma}{\mu} \partial_{x} h \partial_{x}^{3} h
$$

Integration with respect to $y$ then yields

$$
v=\left(\frac{y^{3}}{3}-h y^{2}\right) \frac{\sigma}{2 \mu} \partial_{x}^{4} h-\frac{y^{2}}{2} \frac{\sigma}{\mu} \partial_{x} h \partial_{x}^{3} h
$$

Finally, we must have at the surface of the flow that the convective derivative of $y-h(t, x)$ vanishes, so that on this surface

$$
\partial_{t} h=-u \partial_{x} h+v
$$


in which we substitute the above expressions with $y$ replaced by $h$ to obtain

$$
\partial_{t} h=-h^{2} \frac{\sigma}{\mu} \partial_{x}^{3} h \partial_{x} h-\frac{h^{3}}{3} \frac{\sigma}{\mu} \partial_{x}^{4} h=-\frac{\sigma}{\mu} \partial_{x}\left(h^{3} \partial_{x}^{3} h\right)
$$

This is (1.2) with $n=3$.

\subsection{Degenerate diffusion equations: fourth order ver- sus second order}

We now compare the fourth-order "lubrication approximation" equation (1.2) to the second-order porous media equation

$$
\partial_{t} u=\partial_{x}^{2}\left(u^{\nu}\right)
$$

A qualitative comparison between (7.13) and second-order degenerate parabolic problems of porous media type can be found, for example, in [10]. In essence, the common features exhibited are the parabolicity, the divergence structure and the existence of nonnegative solutions corresponding to nonnegative initial data. The nature of these equations is very much different from their second order analogues in that there is no maximum principle for these higher order equations. As a consequence, (1.2) is analyzed via dissipation of certain nonlinear entropies (defined below).

The porous media equation for $\nu>1$ has unique weak solutions. On the real line, if the initial data is nonnegative and has compact support, then there exists a unique nonnegative weak solution to the equation. This solution has compact support that propagates with a finite speed. The porous media equation possesses nonnegative self-similar source-type solutions for all $\nu>1$ and weak solutions with compact support converge to the source-type solutions as $t \rightarrow \infty$. For $1<\nu<2$ the source-type solutions touch down with zero slope. In [10], the authors found a family of solutions for the fourth-order lubrication equation that also touch down with zero slope. In [10], it was showed that when $1<\nu<2$ and $n>0$ there exists weak solutions to equation (1.2) that have zero slope at the edge. 


\subsection{A definition of entropy}

In this section we will introduce the main idea behind the entropy-entropy dissipation techniques. These techniques are used in order to understand the structure of nonlinear partial differential equations and the qualitative behavior of their solutions. Entropy methods are important tools not only to prove the long-time behavior of solutions to evolution equations. In fact, they may provide a priori estimates, which can be used in proving the global-in-time existence of weak solutions. The entropy methods can substitute the minimum/maximum principle for system of equations. This is of importance since the classical maximum principle for elliptic or parabolic second- order equations generally does not hold in such situations.

Generally speaking, an entropy is a Lyapunov functional of a specific form. It is, however, hard (and even somewhat artificial) to give a formal narrow definition of entropy that distinguishes them from, say, energies. The term "entropy" is frequently used for a Lyapunov functional whose rate of decrease can be bounded in terms of itself. That is, if $H(f)$ is some functional of $f$, and along the flow of some evolution we have:

$$
\frac{d}{d t} H(f) \leq-\Phi(H(f))
$$

with $\Phi$ some continuous strictly monotone increasing function on $\mathbb{R}_{+}$, then the functional $H(f)$ is called an entropy and the inequality (1.4) is called an entropy- entropy dissipation inequality. The point is that (1.4) can be used to quantitatively estimate the rate of decay of $H(f)[43]$.

Apart from the principal types of entropies, we will occasinally consider further functionals in the context of the thin film equation. These functionals are integrals which contain spatial derivatives of $u$; however, we shall refer to them as energies rather than entropies. More 
precisely, we are looking for quantities of the following form as energy

$$
J[u]:=\frac{1}{2} \int_{\mathbb{R}}\left(\partial_{x} u(t, x)\right)^{2} d x
$$

and the following functionals as entropies [42]

$$
H[u]:= \begin{cases}\int_{\mathbb{R}} \frac{u^{2-n}}{(n-1)(n-2)} d x \text { if } n \in(0,1) \cup(1,2) \cup(2, \infty), \\ \int_{\mathbb{R}}(u \log u-u) d x \quad \text { if } n=1 .\end{cases}
$$




\section{Chapter 2}

\section{Existence and regularity results}

\subsection{Existence results for the thin film equations in a bounded domain}

In this section we give an overview of the existence and regularity results for nonnegative weak solutions with zero contact angles for the thin film equation in a bounded domain and present the ideas in [7], [6], and [10] on the construction of a solution of the initial boundary valued problem (2.1) by means of positive smooth approximations. Generally, a thin film equation is a nonlinear, fourth-order, degenerate, parabolic partial differential equation.

Let us consider the equation in one space dimension:

$$
\begin{cases}\partial_{t} u+\partial_{x}\left(|u|^{n} \partial_{x}^{3} u\right)=0 & \text { in } Q=(0, \infty) \times \Omega \\ u(0, x)=u_{0}(x) & \text { for } x \in \Omega:=(-a, a) \\ \partial_{x} u=\partial_{x}^{3} u=0 & \text { for } x=-a \text { and } x=a, t>0\end{cases}
$$

where $\Omega$ is a bounded open interval of $\mathbb{R}, a>0, Q_{T}=\Omega \times(0, T)$, and

$$
u_{0} \in H^{1}(\Omega), u_{0} \not \equiv 0
$$

Assume $u_{0}=0$ in the nonempty open subinterval $\omega=\left(b-r_{0}, b+r_{0}\right)$ of $\Omega$.

The main feature of (2.1) is its degenerate structure, i.e. the ellipticity of the operator on 
the right-hand side is lost where $u$ vanishes. This causes difficulties with the existence theory. However, it is also the origin of an important property of solutions, namely the preservation of non-negativity. Loosely speaking; the degeneracy makes the $x$-axis impenetrable to solutions. It is well known that this positivity result is false for solutions of the linear equation $\partial_{t} u+$ $\partial_{x}^{4} u=0$ (obtained in the limit $n \searrow 0$ ). The questions related to the phenomenon of non-negativity are still of great interest in the theory of thin-film equations. Even in one dimension, it is still not known which is the exact range of $n$ such that thin films do not rupture. Here "rupture" means that the solution $u$ vanishes at some point, $u(\hat{t}, \hat{x})=0$ although it has been strictly positive at this location before, $u(\tau, \hat{x})>0$ for some $\tau<\hat{t}$. The proof of positivity is usually achieved by means of the maximum principle. However, for higher-order equations, this principle generally does not apply. Instead the entropy-entropy dissipation inequalities can be helpful.

Definition 2.1. Let $u: \bar{Q} \rightarrow \mathbb{R}$ be a function such that $u(0, \cdot)=u_{0}$ in $\Omega$ with $u_{0}=0$ in some nonempty open subset of $\Omega$. We say that $u$ has finite speed of propagation if for all $\omega$ satisfying (2.2) there exist a number $T^{*}>0$ and two continuous functions $b_{-}(t), b_{+}(t)$ such that $b_{-}(t)<b_{+}(t)$ in $\left(O, T^{*}\right), b_{-}(0)=b-r_{0}, b_{+}(0)=b+r_{0}$ and $u(t, \cdot)=0$ in $\left(b_{-}(t), b_{+}(t)\right)$ for all $t \in\left(0, T^{*}\right)$.

The boundary of the support, usually called the interface (or free boundary) $\zeta(t)$ of the function $u$ associated to the extreme point $b-r_{0}$ of $\omega$ is defined in the following way.

Definition 2.2. Under the conditions of Definition (2.1), for each $t \in\left[0, T^{*}\right)$ we set

$$
\zeta(t)=\sup \left\{x \in \operatorname{spt}[u(t, \cdot)]: x \leq b_{-}(t)\right\}
$$

if the set considered in (2.3) is nonempty; otherwise we set $\zeta(t)=-a$. We say that $\zeta(t)$ exists if $0 \leq t \leq T^{*}$ for some $T^{*}$ satisfying Definition (2.1).

First we present the concepts of weak and strong solutions as in [7], [6], and [10] because we do not expect to have four derivatives of $u(t, x)$ in the vicinity of the contact line. 
Definition 2.3. Let $n>0$. A weak solution of Problem (2.1) is a function $u$ satisfying the following relations:

$$
\begin{aligned}
& u \in C^{1 / 8,1 / 2}(\bar{Q}) \cap L^{\infty}\left(0, \infty ; H^{1}(\Omega)\right), \\
& u \in C^{1,4}(\mathcal{R}) \text { and }|u|^{n / 2} \partial_{x}^{3} u \in L^{2}(\mathcal{R}),
\end{aligned}
$$

where $\mathcal{R}=\bar{Q} \backslash(\{u=0\} \cup\{t=0\})$, u satisfies (2.1) in the following sense:

$$
\iint_{\mathcal{Q}} u \partial_{t} \varphi d x d t+\iint_{\mathcal{R}} u^{n} \partial_{x}^{3} u \partial_{x} \varphi d x d t=0
$$

for all $\varphi \in \operatorname{Lip}(\bar{Q})$ with compact support in $\bar{\Omega} \times(0, \infty)$;

$$
\begin{gathered}
u(0, \cdot)=u_{0} \text { in } \bar{\Omega}, \\
\partial_{x} u(t, \cdot) \rightarrow \partial_{x} u(0, \cdot) \text { strongly in } L^{2}(\Omega) \text { as } t \rightarrow 0,
\end{gathered}
$$

and

$u$ satisfies the boundary conditions at the points where $u \neq \equiv$.

The boundary of the support of the weak solution physically corresponds to the contact line, the triple contact point of the air/liquid, air/solid, and solid/liquid interfaces (more details in the previous chapter).

A weak solution of Problem (2.1) enjoys the property of mass conservation:

$$
\int_{\Omega} u(t, x) d x=\text { constant }=\int_{\Omega} u_{0}(x) d x \text { for all } t \geq 0
$$

This follows by taking in $(2.6) \varphi=\Theta(t)$ with $\Theta \in C_{c}^{1}((0, \infty))$. Notice that $\varphi$ may be different from zero at the lateral boundary of the cylinder $\bar{\Omega} \times(0, \infty)$.

Recall: $u \in C^{1 / 8,1 / 2}(\bar{Q}): u \in C(\bar{Q}) \cap L^{\infty}(Q)$ and there exists a constant $M$ such that for all $x, y \in \bar{\Omega}$ and all $t, T \in[0, \infty)|u(t, x)-u(t, y)| \leq M|x-y|^{1 / 2}$ and $|u(T, x)-u(t, x)| \leq$ $M|T-t|^{1 / 8}$.

$u \in C^{1,4}(\mathcal{R})$, where $\mathcal{R} \subset \bar{Q}: u, u_{t}$ and all the spatial derivatives up to fourth order are continuous in $\mathcal{R}$. 
Remark 2.4. A weak solution u of Problem (2.1) satisfies $u \in C^{\infty}(\mathcal{R})$, since the function $f(s)=|s|^{n}$ is $C^{\infty}$ for $s \neq 0$, and from (2.5)-(2.6) and linear parabolic theory.

Definition 2.5. A strong solution of Problem (2.1) is a week solution u such that $u(t, \cdot) \in$ $C^{1}(\bar{\Omega})$ for almost every $t>0$ (see [5], [7]).

Notice that strong solutions are weaker than classical solutions.

Since (2.1) is degenerate at $u=0$, we begin by approximating it by a family of nondegenerate diffusions:

$$
\begin{cases}\partial_{t} u_{\varepsilon}+\partial_{x}\left(\left(f\left(u_{\varepsilon}\right)+\varepsilon\right) \partial_{x}^{3} u_{\varepsilon}\right)=0 & \text { in } Q \\ u(0, x)=u_{0 \varepsilon}(x) & \text { for } x \in \Omega \\ \partial_{x} u_{\varepsilon}=\partial_{x}^{3} u_{\varepsilon}=0 & \text { for } x=-a \text { and } x=a, t>0 .\end{cases}
$$

where $f(u)=|u|^{n} f_{0}(u), \quad 0<f_{0} \in C^{1, \alpha}(\mathbb{R}), \alpha \in(0,1)$.

Proposition 2.6. (Global existence of smooth solution for the regularized problem [7]) Let $0 \leq u_{0} \in H^{1}(\Omega), u_{0} \neq 0, n \geq 1$ and $\varepsilon>0$. The approximating problem above has a unique positive solution

$$
u_{\varepsilon} \in C^{1 / 8,1 / 2}(\bar{Q}) \cap C^{\infty}(\bar{\Omega} \times(0, \infty))
$$

such that $u_{\varepsilon}$ satisfies (2.8).

Proof. Using the classical parabolic Schauder estimates one can prove existence of a smooth solution up to a time $s$, say in $Q_{s}$ for some small $s>0$. The derivatives

$$
\partial_{t} u_{\varepsilon}, \partial_{x} u_{\varepsilon}, \partial_{x}^{2} u_{\varepsilon}, \partial_{x}^{3} u_{\varepsilon}, \partial_{x}^{4} u_{\varepsilon}
$$

are all Hölder continuous in $\overline{Q_{s}}$. Now we assume that $u_{\varepsilon}$ is a solution in $Q_{s}$, for some $0<s<T$ and derive various estimates. We begin with

$$
\begin{gathered}
\int_{\Omega}\left[\left(\partial_{x} u_{\varepsilon}(t+h, x)\right)^{2}-\left(\partial_{x} u_{\varepsilon}(t, x)\right)^{2}\right] d x=\int_{\Omega}\left[\partial_{x} u_{\varepsilon}(t+h, x)-\partial_{x} u_{\varepsilon}(t, x)\right]\left[\partial_{x} u_{\varepsilon}(t+h, x)+\partial_{x} u_{\varepsilon}(t, x)\right] d x \\
=-\int_{\Omega}\left[\partial_{x}^{2} u_{\varepsilon}(t+h, x)+\partial_{x}^{2} u_{\varepsilon}(t, x)\right]\left[u_{\varepsilon}(t+h, x)-u_{\varepsilon}(t, x)\right] d x
\end{gathered}
$$


since $\partial_{x} u=0$ on the boundary. Dividing by $h$ and letting $h \rightarrow 0$ we get

$$
\int_{\Omega} \partial_{t}\left(\partial_{x} u_{\varepsilon}(t, x)\right)^{2} d x=-2 \int_{\Omega} \partial_{x}^{2} u_{\varepsilon}(t, x) \partial_{t} u(t, x) d x
$$

then integrate both sides with respect to time for any $0<t_{1}<t_{2}<s$,

$$
\left[\int_{\Omega}\left(\partial_{x} u_{\varepsilon}(t, x)\right)^{2} d x\right]_{t=t_{1}}^{t=t_{2}}=-2 \int_{t=t_{1}}^{t=t_{2}} \int_{\Omega} \partial_{x}^{2} u_{\varepsilon}(t, x) \partial_{t} u_{\varepsilon}(t, x) d x d t
$$

Now multiply (2.10) by $\partial_{x}^{2} u_{\varepsilon}$ and integrate over $Q_{T_{0}}\left(0<T_{0}<s\right)$

$$
\int_{0}^{T_{0}} \int_{\Omega} \partial_{t} u_{\varepsilon} \partial_{x}^{2} u_{\varepsilon} d x d t-\int_{0}^{T_{0}} \int_{\Omega}\left(f\left(u_{\varepsilon}\right)+\varepsilon\right)\left(\partial_{x}^{3} u_{\varepsilon}(t, x)\right)^{2} d x d t=0,
$$

and using the last identity, we get

$$
\frac{1}{2} \int_{\Omega}\left(\partial_{x} u_{\varepsilon}\left(T_{0}, x\right)\right)^{2} d x+\int_{0}^{T_{0}} \int_{\Omega}\left(f\left(u_{\varepsilon}\right)+\varepsilon\right)\left(\partial_{x}^{3} u_{\varepsilon}(t, x)\right)^{2} d x d t=\frac{1}{2} \int_{\Omega}\left(\partial_{x} u_{0 \varepsilon}\right)^{2} d x
$$

Hence

$$
\int_{\Omega}\left(\partial_{x} u_{\varepsilon}\left(T_{0}, x\right)\right)^{2} d x \leq \int_{\Omega}\left(\partial_{x} u_{0 \varepsilon}\right)^{2} d x
$$

Integrate (2.10) over $Q_{T_{0}}$ to get

$$
\int_{\Omega} u_{\varepsilon}\left(T_{0}, x\right) d x=\int_{\Omega} u_{0 \varepsilon} d x
$$

Notice that

$$
\int_{\Omega}\left(\partial_{x} u_{0 \varepsilon}\right)^{2} d x \leq(1+g(\varepsilon)) \int_{\Omega}\left(\partial_{x} u_{0}\right)^{2} d x
$$

and $g(\varepsilon) \rightarrow 0$ if $\varepsilon \rightarrow 0$, which guarantees an upper bound, independent of $T_{0}$, for $\int_{\Omega} \partial_{x} u_{\varepsilon}\left(T_{0}, x\right) d x$ for any time $T_{0}<s$. From (2.13), (2.14) we deduce, by the Poincare' inequality, that

$$
\left|u_{\varepsilon}(t, x)\right| \leq C \text { in } Q_{s}
$$

where $C$ is a constant independent of $\varepsilon, s$. From $(2.13),(2.15)$ and Sobolev's inequality we also deduce that

$$
\left|u_{\varepsilon}\left(t, x_{2}\right)-u_{\varepsilon}\left(t, x_{1}\right)\right| \leq K\left|x_{2}-x_{1}\right|^{1 / 2} \text { in } Q_{s}
$$


where $K$ a constant independent of $\varepsilon, s$. Setting

$$
h_{\varepsilon}=\left(f\left(u_{\varepsilon}\right)+\varepsilon\right) \partial_{x}^{3} u_{\varepsilon} .
$$

From (2.12), (2.16) we have

$$
\int_{0}^{T_{0}} \int_{\Omega}\left|h_{\varepsilon}(t, x)\right|^{2} d x d t \leq C_{1}
$$

where $C_{1}$ is a constant independent of $\varepsilon, s$. One can use (2.16), (2.17) to prove that there exists an upper bound $M$ independent of $\varepsilon, s$ such that

$$
\left|u_{\varepsilon}\left(t_{2}, x\right)-u_{\varepsilon}\left(t_{1}, x\right)\right| \leq M\left|t_{2}-t_{1}\right|^{1 / 8}
$$

for all $x \in \Omega, t_{1}$ and $t_{2}$ in $(0, s)$. To prove the Hölder continuity in $t$ we suppose on the contrary that

$$
\left|u_{\varepsilon}\left(t_{2}, x_{0}\right)-u_{\varepsilon}\left(t_{1}, x_{0}\right)\right|>M\left|t_{2}-t_{1}\right|^{\beta}
$$

for some $x_{0}$ and $t_{2}, t_{1}$. To complete the proof we will derive an upper bound on $M$ that is independent of $\varepsilon, s$. Without loss of generality we suppose that $u_{\varepsilon}\left(t_{2}, x_{0}\right)>u_{\varepsilon}\left(t_{1}, x_{0}\right)$ and $t_{2}>t_{1}$. Hence

$$
u_{\varepsilon}\left(t_{2}, x_{0}\right)-u_{\varepsilon}\left(t_{1}, x_{0}\right)>M\left(t_{2}-t_{1}\right)^{\beta},
$$

where $0<t_{1}<t_{2}<s$. Notice that $u_{\varepsilon}$ satisfies

$$
\iint_{Q_{s}} u_{\varepsilon} \partial_{t} \varphi d x d t=-\iint_{Q_{s}} h_{\varepsilon} \partial_{x} \varphi d x d t
$$

where $\varphi$ is a reasonable test function. Since $\partial_{t} u_{\varepsilon}$ is continuous in $\bar{Q}_{s}$ and $h_{\varepsilon}=0$ on the lateral boundary, we take $\varphi$ such that $\varphi \in \operatorname{Lip}\left(\bar{Q}_{s}\right), \varphi=0$ near $t=0$ and near $t=s$. Note also that $\varphi$ is not necessarily 0 on the lateral boundary. We define $\varphi$ by

$$
\varphi(t, x)=\psi(x) \theta_{\delta}(t)
$$

where $\psi$ and $\theta_{\delta}$ are defined as follows: 
Definition of $\psi$ :

$$
\psi(x)=\psi_{0}\left(\frac{x-x_{0}}{\frac{M^{2}}{16 K^{2}}\left(t_{2}-t_{1}\right)^{2 \beta}}\right)
$$

where $M$ is from (2.20) and $K$ from (2.17). Moreover, the function $\psi_{0}(x)$ satisfies $\psi_{0}(x)=$ $\psi_{0}(-x), \psi_{0} \in C_{0}^{\infty}, \psi_{0}(x)=1$ if $0 \leq x \leq \frac{1}{2}, \psi_{0}(x)=0$ if $x \geq 1, \psi_{0}^{\prime}(x) \leq 0$ if $x \geq 0$.

Notice that we have

$$
\psi(x)= \begin{cases}0 & \text { if }\left|x-x_{0}\right| \geq \frac{M^{2}}{16 K^{2}}\left(t_{2}-t_{1}\right)^{2 \beta} \\ 1 & \text { if }\left|x-x_{0}\right| \leq \frac{1}{2} \frac{M^{2}}{16 K^{2}}\left(t_{2}-t_{1}\right)^{2 \beta}\end{cases}
$$

Definition of $\theta_{\delta}$ : We take

$$
\theta_{\delta}(t)=\int_{-\infty}^{t} \theta_{\delta}^{\prime}(\tau) d \tau
$$

where

$$
\theta_{\delta}^{\prime}(t)= \begin{cases}\frac{1}{\delta} & \text { if }\left|t-t_{2}\right|<\delta \\ -\frac{1}{\delta} & \text { if }\left|t-t_{1}\right|<\delta \\ 0 & \text { elsewhere, }\end{cases}
$$

where $\delta<\frac{1}{2}\left(t_{2}-t_{1}\right)$. Notice that $\theta_{\delta}$ is Lipschitz continuous and $\left|\theta_{\delta}\right| \leq 1 ; \theta_{\delta}=0$ near $t=0$ and near $t=s$, if $\delta$ is small enough. Plugging this function $\varphi(t, x)$ into $(2.21)$ yields that

$$
\iint_{Q_{s}} u_{\varepsilon} \psi(x) \theta_{\delta}^{\prime}(t) d x d t=-\iint_{Q_{s}} h_{\varepsilon} \psi^{\prime}(x) \theta_{\delta}(t) d x d t
$$

As $\delta \rightarrow 0$ the left hand side of this equality satisfies

$$
\iint_{Q_{s}} u_{\varepsilon} \psi(x) \theta_{\delta}^{\prime}(t) d x d t \rightarrow \int_{\Omega} \psi(x)\left(u_{\varepsilon}\left(t_{2}, x\right)-u_{\varepsilon}\left(t_{1}-x\right)\right) d x .
$$

We will find a lower bound for this last expression. By the definition of $\psi(x)$ we only need to consider

$$
\left|x-x_{0}\right| \leq \frac{M^{2}}{16 K^{2}}\left(t_{2}-t_{1}\right)^{2 \beta} .
$$


For such values of $x$, we obtain that

$$
\begin{aligned}
u_{\varepsilon}\left(t_{2}, x\right)-u_{\varepsilon}\left(t_{1}-x\right) & =\left[u_{\varepsilon}\left(t_{2}, x\right)-u_{\varepsilon}\left(t_{2}, x_{0}\right)\right] \\
& +\left[u_{\varepsilon}\left(t_{2}, x_{0}\right)-u_{\varepsilon}\left(t_{1}, x_{0}\right)\right]+\left[u_{\varepsilon}\left(t_{1}, x_{0}\right)-u_{\varepsilon}\left(t_{1}, x\right)\right] \\
& \geq-2 K\left|x-x_{0}\right|^{1 / 2}+M\left(t_{2}-t_{1}\right) \\
& \geq \frac{M}{2}\left(t_{2}-t_{1}\right)^{\beta} .
\end{aligned}
$$

Assume without loss of generality that the set $\psi=1$ is included in $\Omega$. Then

$$
\int_{\Omega} \psi(x)\left(u_{\varepsilon}\left(t_{2}, x\right)-u_{\varepsilon}\left(t_{1}-x\right)\right) d x \geq \frac{M}{2}\left(t_{2}-t_{1}\right)^{\beta} \frac{M^{2}}{16 K^{2}}\left(t_{2}-t_{1}\right)^{2 \beta} .
$$

On the other hand, the right hand side of (2.24) can be bounded from above by means of the Hölder's inequality and definitions of $\psi$ and $\theta_{\delta}$. Indeed, we have

$$
\left|\iint_{Q_{s}} h_{\varepsilon} \psi^{\prime}(x) \theta_{\delta}(t) d x d t,\right| \leq \frac{C_{2}}{\frac{M^{2}}{16 K^{2}}\left(t_{2}-t_{1}\right)^{2 \beta}}\left(\iint_{Q_{s}} h_{\varepsilon}^{2}\right)^{1 / 2} \frac{\sqrt{2} M}{4 k}\left(t_{2}-t_{1}\right)^{\beta}\left(t_{2}-t_{1}+2 \delta\right)^{1 / 2} .
$$

Therefore, by letting $\delta \rightarrow 0$, we deduce that

$$
M^{3}\left(t_{2}-t_{1}\right)^{3 \beta} \leq C_{3} \frac{1}{M}\left(t_{2}-t_{1}\right)^{1 / 2-\beta}
$$

where $C_{3}$ is a constant independent of $\varepsilon, M$ and $s$. Since $\beta=1 / 8$, we have $M \leq C_{3}^{1 / 4}$. Thus

$$
\left|u_{\varepsilon}\left(t_{2}, x\right)-u_{\varepsilon}\left(t_{1}, x\right)\right| \leq M\left|t_{2}-t_{1}\right|^{1 / 8}
$$

It follows that from $(2.16),(2.17)$ and $(2.19)$ there is an upper bound on the $C^{1 / 8,1 / 2}\left(Q_{s}\right)$ norm of $u_{\varepsilon}$ in $Q_{s}$, which is independent of $\varepsilon, s$. This a priori bound allows us to extend $u_{\varepsilon}$ step-by-step to a solution of (2.10) in all of $Q_{T}$, and to conclude that

$$
\left\{u_{\varepsilon}\right\} \text { is a uniformly bounded and equi-cointinous family in } \overline{Q_{T}} \text {. }
$$

The Arzela- Ascoli theorem guarantees that a subsequence converges pointwise uniformly to a limit $u$. 
More specifically, we present the following proposition.

Proposition 2.7. (Existence of Weak Solution for the boundary valued problem (2.1) [7]): Under the conditions of Proposition (2.6), there exists $u \in C^{1 / 8,1 / 2}\left(\bar{Q}_{T}\right)$ and a sequence $\{\varepsilon\}, \varepsilon \rightarrow 0$, such that

$$
u_{\varepsilon} \rightarrow u \text { in } C^{1 / 8,1 / 2}\left(\bar{Q}_{T}\right)
$$

for all $T>0$ and any limit function $u$ obtained in this way is a weak solution of Problem (2.1). Furthermore, the function $u$ is a strong solution if $0<n<3$.

Proof. First $u \in C^{1 / 8,1 / 2}\left(\bar{Q}_{T}\right)$ and $u(0, x)=u_{0}(x)$ are obvious.

$$
\iint_{Q_{T}} u_{\varepsilon} \partial_{t} \varphi d x d t+\iint_{Q_{T}} f\left(u_{\varepsilon}\right) \partial_{x}^{3} u_{\varepsilon} \partial_{x} \varphi d x d t+\varepsilon \iint_{Q_{T}} \partial_{x}^{3} u_{\varepsilon} \partial_{x} \varphi d x d t=0
$$

for all $\varphi \in \operatorname{Lip}\left(\bar{Q}_{T}\right), \varphi=0$ near $t=0$ and near $t=T$. From (2.12),

$$
\varepsilon \iint_{Q_{T}}\left(\partial_{x}^{3} u_{\varepsilon}\right)^{2} d x d t \leq C
$$

hence, by Hölder's inequality,

$$
\varepsilon \iint_{Q_{T}} \partial_{x}^{3} u_{\varepsilon} \partial_{x} \varphi d x d t \rightarrow 0 \text { if } \varepsilon \rightarrow 0
$$

From (2.18) it follows that, for a subsequence,

$$
h_{\varepsilon} \rightarrow h \text { weakly in } L^{2}\left(Q_{T}\right)
$$

By regularity theory of uniformly parabolic equations and uniform Hölder continuity of the $u_{\varepsilon}$ we deduce that $\partial_{t} u_{\varepsilon}, \partial_{x} u_{\varepsilon}, \partial_{x}^{2} u_{\varepsilon}, \partial_{x}^{3} u_{\varepsilon}, \partial_{x}^{4} u_{\varepsilon}$ are uniformly convergent in any compact subset of $\mathcal{R}$. It follows that $f(u) \partial_{x}^{3} u=h$ on $\mathcal{R}$, that $u \in C^{1,4}(\mathcal{R})$ and (2.9) hold. Also, by (2.29) we have $f(u) \partial_{x}^{3} u \in L^{2}(\mathcal{R})$; for any $s>0$

$$
\iint_{|u|>s} f\left(u_{\varepsilon}\right) \partial_{x}^{3} u_{\varepsilon} \partial_{x} \varphi d x d t \rightarrow \iint_{|u|>s} f(u) \partial_{x}^{3} u \partial_{x} \varphi d x d t
$$


On the other hand, if $\varepsilon$ is sufficiently small, depending on $s$, then by

$$
\begin{array}{r}
\left|\iint_{|u| \leq s} f\left(u_{\varepsilon}\right) \partial_{x}^{3} u_{\varepsilon} \partial_{x} \varphi d x d t\right|=\left|\iint_{|u| \leq s} f\left(u_{\varepsilon}\right)^{1 / 2} f\left(u_{\varepsilon}\right)^{1 / 2} \partial_{x}^{3} u_{\varepsilon} \partial_{x} \varphi d x d t\right| \\
\leq C s^{n / 2}\left\{\iint_{|u| \leq s} f\left(u_{\varepsilon}\right)\left(\partial_{x}^{3} u_{\varepsilon}\right)^{2} d x d t\right\}^{1 / 2} \leq C_{1} s^{1 / 2}
\end{array}
$$

For the first inequality we used that $f(u)=|u|^{n} f_{0}(u)$ where $f_{0} \in C^{1, \alpha}(\mathbb{R}), \alpha \in(0,1)$ and the Hölder inequality, and for the second one we used (2.12). Notice that from $u_{0 \varepsilon} \rightarrow u_{0}$ in $H^{1}(\Omega)$ and $(2.13)$ we get

$$
\limsup _{t \rightarrow 0} \int_{\Omega}\left(\partial_{x} u(t, x)\right)^{2} d x \leq \int_{\Omega}\left(\partial_{x} u_{0}\right)^{2} d x
$$

since also $\partial_{x} u(t, \cdot) \rightarrow \partial_{x} u_{0}$ weakly in $L^{2}(\Omega)$ as $t \rightarrow 0$ implies $\partial_{x} u(t, \cdot) \rightarrow \partial_{x} u_{0}$ strongly in $L^{2}(\Omega)$ as $t \rightarrow 0$. Taking $\varepsilon \rightarrow 0$ in (2.27) and using (2.28), (2.30), (2.31) we deduce, since $s$ is arbitrary, that (2.6) is satisfies.

Remark 2.8. 1) Note that by passing to the limit as $\varepsilon \rightarrow 0$ we obtained a weak solution of the (2.1) for $n \geq 1$ [7]. The paper [10] extended this result to all $n>0$ and proved that the solution is strong if $0<n<3$.

2) We can construct a weak solution using also other approximations to $f(s)$ and $u_{0}$, namely

$$
f_{\varepsilon}(s)=\frac{s^{4} f(s)}{\varepsilon f(s)+s^{4}}, \quad u_{0 \varepsilon}(x)=u_{0}(x)+\varepsilon^{\theta}, \quad \text { for some } 0<\theta<\frac{2}{5} .
$$

Note that $f_{\varepsilon}$ is still degenerate; however if $0<n<4$, then $f_{\varepsilon} \sim \frac{s^{4}}{\varepsilon}$ for small $s>0$, [y] [10].

\section{Finite speed of propagation}

We consider the interface $\zeta(t)$ (Definition (2.1)) of the solution $u$ associated to the extreme $b-r_{0}$ of $\omega$ as in Definition (2.2). Without loss of generality we assume that

$$
\zeta(0)=b-r_{0}>-a, \text { that } b-r_{0} \in \operatorname{spt} u_{0} .
$$

Proposition 2.9. (Finite speed of propagation [6])

Let $0<n<2$. Assume that $u_{0}=0$ in a nonempty open subset of $\Omega$. Then the nonnegative 
strong solution u of Problem (2.1) defined in Proposition (2.7) has finite speed of propagation in the sense of Definition (2.1). Furthermore, let $\lambda$ satisfy $\max \{-1 / 2, n-1\}<\lambda<1$. Then there exists a positive constant $T^{*}=T^{*}\left(\lambda, n, u_{0}, r_{0}\right)$ such that if $0<T<T^{*}$ the interface $\zeta(T)$ of Definition (2.1) exists and

$$
\zeta(T)-\zeta(0) \leq A_{0} T^{\alpha}\left(\int_{0}^{T} \int_{\zeta(0)}^{b} \partial_{x}^{2}\left(u^{(\lambda+2) / 2}\right)^{2} d x d t\right)^{\beta}
$$

where $A_{0}$ is a positive constant depending only on $\lambda$ and $n$ and $\alpha=\frac{2-n+\lambda}{8-3 n+4 \lambda}, \beta=\frac{n}{8-3 n+4 \lambda}$.

Now, on physical grounds, we have

$$
u_{0} \geq 0 \rightarrow u(t, x) \geq 0, \quad \forall t>0, \forall x \in \Omega
$$

which is shown in [7] by employing entropy dissipation methods.

\subsection{Existence results for the Cauchy problem}

In [6], the author shows that strong solutions for the natural boundary-value problem (first and third derivatives are zero at the boundary) can be used to construct a strong solution for the Cauchy problem. These solutions are obtained as accumulation points in the appropriate topology of classical solutions for regularized problems (2.10). Set

$$
\zeta(t)=\sup \operatorname{spt}[u(t, \cdot)], \zeta_{-}(t)=\inf \operatorname{spt}[u(t, \cdot)], \quad|\zeta(t)|=\zeta(t)-\zeta_{-}(t) .
$$

Set $\mathcal{Q}:=(0, \infty) \times \mathbb{R}, \mathcal{R}:=\{u>0\} \cap\{t>0\}$ and consider

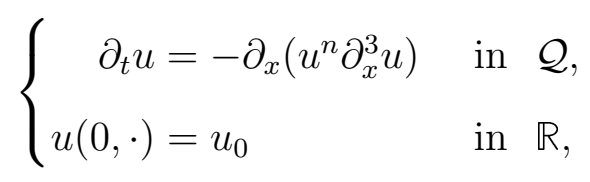

where

$u_{0} \in H^{1}(\mathbb{R}), u_{0} \geq 0, u_{0} \not \equiv 0$, and the support $u_{0}$ is compact. 
The definitions of weak solution and strong solution of Problem (2.38) are as Definitions (2.3), (2.5) except that $\Omega$ is replaced by $\mathbb{R}$ (also $\bar{\Omega}=\mathbb{R}$ ) and the relation (2.9) is dropped. Let $0<n<2$. Given $T>0$, let $\hat{u}$ be the solution of the Problem (2.1) defined in Proposition (2.7) and choose $a$ so that $-a<\zeta_{-}(t)<\zeta(t)<a$ for all $t \in[0, T][6]$.

For $t \in[0, T]$ we set

$$
u(t, x)= \begin{cases}\hat{u}(t, x) & \text { if } \zeta_{-}(t) \leq x \leq \zeta(t) \\ 0 & \text { if } x<\zeta_{-}(t) \text { or } x>\zeta(t)\end{cases}
$$

Performing a similar construction in $[T, 2 T], \cdots,[m T,(m+1) T], \cdots$, we obtain a nonnegative strong solution $u$ of (2.38) that satisfies the same identities and estimates as the solution of Problem (2.1) and clearly $u(t, x)$ has compact support for all $t \geq 0$. Notice that the estimates depending on $a$ for Problem (2.1) may depend on $T$ for Problem (2.38).

\subsubsection{Our result}

We assume $u_{0} \in H^{1}(\mathbb{R})$ and $u_{0} \geq 0$ of positive total mass $m$. The reference [6] is where the Cauchy problem for compactly supported such $u_{0}$ was studied in detail. The definitions and properties we give below for solutions stemming from compactly supported initial data are taken from [6], [10].

Definition 2.10. A weak solution for (2.38) with initial nonnegative $u_{0} \in H^{1}(\mathbb{R})$ is a nonnegative function $u(t, x)$ such that:

$$
\begin{gathered}
u \in L^{\infty}\left(0, \infty ; H^{1}(\mathbb{R})\right), \\
u \in C^{\infty}(\mathcal{R}), u^{n} \partial_{x}^{3} u^{2} \in L^{1}(\mathcal{R})
\end{gathered}
$$

and

$$
\iint_{\mathcal{Q}} u \partial_{t} \varphi d x d t+\iint_{\mathcal{R}} u^{n} \partial_{x}^{3} u \partial_{x} \varphi d x d t=0
$$

for all $\varphi \in \operatorname{Lip}((0, \infty) \times \mathbb{R})$ with compact support inside $\mathcal{Q}$. Also,

$$
u(t, \cdot) \rightarrow u_{0} \quad \text { as } \quad t \downarrow 0 .
$$


Notice that we do not require, as in [6], [13], that the weak solutions satisfy $u \in$ $C^{1 / 8,1 / 2}((0, \infty) \times \mathbb{R})$. Also, we do not ask that $u(0, \cdot)=u_{0}$ pointwise and $\partial_{x} u(t, \cdot) \rightarrow \partial_{x} u_{0}$ in $L^{2}(\mathbb{R})$ as $t \downarrow 0$.

Our main decay result is proved for solutions enjoying some extra properties.

Definition 2.11. A dissipative mild solution u for (2.38) is a weak solution as in Definition 2.10 that satisfies the extra conditions

$$
u(t, \cdot) \in H^{2}(\mathbb{R}) \text { for almost all } t>0,
$$

and

$$
J[u(t, \cdot)]+\int_{s}^{t} \int_{\mathcal{P}_{\tau}} u^{n}(\tau, x)\left(\partial_{x}^{3} u(\tau, x)\right)^{2} d x d \tau \leq J[u(s, \cdot)] \quad \text { for almost all } 0<s \leq t .
$$

where $\mathcal{P}=\{x \in \mathbb{R} \mid u(t, x)>0\}$.

Although weaker than classical solutions, there is the concept of strong solutions.

Definition 2.12. A dissipative strong solution u for (2.38) is a dissipative mild solution as in Definition 2.11 that satisfies the extra conditions

$$
u \in C^{1 / 8,1 / 2}([0, \infty) \times \mathbb{R}) \cap L^{2}\left(0, T ; H^{2}(\mathbb{R})\right)
$$

and

$$
u(0, x)=u_{0}(x) \text { for all } x \in \mathbb{R} \quad \text { and } \quad \partial_{x} u(t, \cdot) \rightarrow \partial_{x} u_{0} \quad \text { in } \quad L^{2}(\mathbb{R}) \text { as } t \downarrow 0 \text {. }
$$

For compactly supported initial data, strong solutions were obtained in [6], [7], [10]. In the case $n=1$, Carrillo and Toscani [13] showed that the compactness of support restriction may be eliminated by approximation. We will mimic their approach here to prove Theorem 2.13. Before that, let us recall [6], [10] the following entropy functionals (significant for various values of $n$ )

$$
H[u]:= \begin{cases}\int_{\mathbb{R}} \frac{u^{2-n}}{(n-1)(n-2)} d x & \text { if } n \in(0, \infty) \backslash\{1,2\} \\ \int_{\mathbb{R}}(u \log u-u) d x & \text { if } n=1 .\end{cases}
$$


It turns out that, for fixed $T>0$, the dissipation of $H$ along a strong solution $u$ is bounded from below by the square of the $L^{2}((0, T) \times \mathbb{R})-$ norm of $\partial_{x}^{2} u$.

Theorem 2.13. (Main result)

Let $u \in H^{1}(\mathbb{R})$ be nonnegative of unit total mass, finite second-order moment, and such that $H\left[u_{0}\right]<\infty$. If $\frac{3}{8}<n<2$, then there exists a dissipative strong solution for (2.38).

Proof. We consider a sequence of smooth cut-off functions $0 \leq \xi_{k} \leq 1$ such that $\xi_{k} \equiv 0$ outside $(-k-1, k+1), \xi_{k} \equiv 1$ in $[-k, k]$ and $\left|\xi_{k}^{\prime}\right| \leq 2$ on $\mathbb{R}$. Consider now $u_{0 k}:=\xi_{k} u_{0}$ and note that the mass $m_{k}$ of $u_{0 k}$ tends to 1 as $k \rightarrow \infty$ (in particular, $m_{k}>0$ for large enough $k)$. It is easy to see that

$$
u_{0 k} \rightarrow u_{0} \quad \text { in } \quad L^{1}(\mathbb{R}) \cap H^{1}(\mathbb{R}) \quad \text { as } \quad k \rightarrow \infty .
$$

For all sufficiently large $k$ let $u_{k}$ be the strong solutions constructed in [6]. We will prove they all satisfy

$$
J\left[u_{k}(t, \cdot)\right]+\int_{0}^{t} \int_{\mathcal{P}_{k, \tau}} u_{k}^{n}(\tau, x)\left(\partial_{x}^{3} u_{k}(\tau, x)\right)^{2} d x d \tau \leq J\left[u_{0 k}\right]
$$

where $\mathcal{P}_{k, t}:=\left\{x \in \mathbb{R}: u_{k}(t, x)>0\right\}$. Also,

$$
\int_{\mathbb{R}} u_{k}(t, x) d x=\int_{\mathbb{R}} u_{0 k}(x) d x=m_{k} \leq 1 \quad \text { for } t \geq 0
$$

But

$$
J\left[u_{0 k}\right]=\frac{1}{2}\left\|\partial_{x} \xi_{k} u_{0}+\xi_{k} \partial_{x} u_{0}\right\|_{L^{2}(\mathbb{R})}^{2} \leq 2\left\{2\left\|u_{0}\right\|_{L^{2}(\mathbb{R})}^{2}+J\left[u_{0}\right]\right\},
$$

which, in particular, implies that $u_{k}$ are uniformly essentially bounded in $\mathcal{Q}$. Define $h_{k}:=$ $u_{k}^{n} \partial_{x}^{3} u_{k}$ on $\mathcal{R}_{k}$ and $h_{k}:=0$ otherwise. The uniform $L^{\infty}$ bound on $u_{k},(2.46)$ and (2.47) imply

$$
h_{k} \text { is uniformly bounded in } L^{2}(\mathcal{Q}) \text {. }
$$

Also, (2.42) implies

$$
\iint_{\mathcal{Q}} u_{k} \partial_{t} \varphi d x d t+\iint_{\mathcal{Q}} h_{k} \partial_{x} \varphi d x d t=0
$$


for all $\varphi \in \operatorname{Lip}([0, \infty) \times \mathbb{R})$ with compact support inside $\mathcal{Q}$. One immediate consequence is that

$$
\partial_{t} u_{k} \text { is uniformly bounded in } L^{2}\left(0, T ; H^{-1}(\mathbb{R})\right) \text { for all } T>0 \text {. }
$$

The inequality [7], [10]

$$
\int_{0}^{T} \int_{\mathbb{R}} \partial_{x}^{2} u_{k}^{2}(t, x) d x d t \leq H\left[u_{0 k}\right]-H\left[u_{k}(T, \cdot)\right] \text { for all } T>0
$$

together with the uniform bound on the right hand side (see, e.g., [42]) yields that $u_{k}$ is uniformly bounded in $L^{2}\left(0, T ; H^{2}(\mathbb{R})\right)$ for any $T>0$. Therefore, in light of $(2.51)$, there exists a subsequence (not relabelled) and $u \in L^{2}\left(0, T ; H^{2}(\mathbb{R})\right)$ such that

$$
u_{k} \rightarrow u \text { weakly in } L^{2}\left(0, T ; H^{2}(\mathbb{R})\right) \text { and strongly in } L^{2}\left(0, T ; H^{1}(\mathbb{R})\right) \text { for all } T>0,(2.52)
$$

where the strong convergence is due to a celebrated result of Lions-Aubin (see, e.g., [10]).

Now we specialize to two cases:

Case 1: $\frac{3}{8}<n \leq 1$. We conclude exactly as in [13] by using equations (5.3) and (5.4) in said reference. Note that the arguments in [13] are valid for $n$ within this range and not just for $n=1$. Indeed, the equations (5.3) and (5.4) were taken by the authors of [13] from [10], were they are showed to be valid within this range for $n$.

Case 2: $1<n<2$. In this case, the solutions $u_{k}$ satisfy [10]

$$
\iint_{\mathcal{Q}} u_{k} \partial_{t} \phi d x d t=\iint_{\mathcal{Q}} u_{k}^{n} \partial_{x}^{2} u_{k} \partial_{x}^{2} \phi d x d t+n \iint_{\mathcal{Q}} u_{k}^{n-1} \partial_{x} u_{k} \partial_{x}^{2} u_{k} \partial_{x} \phi d x d t
$$

for any $\phi \in C_{c}^{\infty}(\mathcal{Q})$. In light of $(2.47)$ and as in [11] we obtain that

$$
u_{k} \text { is uniformly bounded in the sup norm over } \mathcal{Q} \text {. }
$$

Furthermore, the uniform $H^{1}(\mathbb{R})$ bound on $u_{k}(t, \cdot)$ (from $(2.47)$ ) provides a constant $K>0$ such that

$$
\left|u_{k}(t, x)-u_{k}(t, y)\right| \leq K|x-y|^{1 / 2} \quad \text { in } \mathcal{Q} \text {. }
$$

In light of $(2.50),(2.55),(2.49)$ and the last displayed equation, we are now in the position 
to argue as in [7] (Lemma 2.1) (by using special "localized" test functions) and prove that there exists $K^{\prime}>0$ (independent of $k$ ) such that

$$
\left|u_{k}(t, x)-u_{k}(s, x)\right| \leq K^{\prime}|t-s|^{1 / 8} \quad \text { in } \mathcal{Q} \text {. }
$$

By Ascoli-Arzela's Theorem, (2.49), (2.55) and (2.56) give the existence of a subsequence (not relabeled) $u_{k}$ converging to $u$ locally uniformly in $\mathcal{Q}$. Thus, for $n>1$ we have that $u_{k}^{n-1} \rightarrow u^{n-1}$ locally uniformly in $\mathcal{Q}$. In conjunction with $(2.52)$, this shows that we can pass to the limit in (2.53) to conclude that $u$ is a strong solution for (2.38).

To finish the proof, we need to prove that dissipation property $(2.45)$, for any $3 / 8<n<2$. We will do this in two steps: first we show that it holds for the approximations $u_{k}$ and then we pass to the limit to get it for $u$. To avoid multi-indexing, let us consider a fixed $k$ and set $v:=u_{k}$. Fix $T>0$ and $a \in \mathbb{R}$ sufficiently large so that the support of $v(t, \cdot)$ lies within $(-a, a)$ for all $0 \leq t \leq T$. Next, consider the smooth approximation $v_{\varepsilon}$ recalled in $(0.3)$. We have that, at least up to a subsequence, $v_{\varepsilon}$ converges [6], [10] to $v$ in $L^{2}\left(0, T ; H^{1}((-a, a))\right)$. The uniform $L^{2}((0, T) \times(-a, a))$-bound on $w_{\varepsilon}:=\left[f_{\varepsilon}\left(v_{\varepsilon}\right)\right]^{1 / 2} \partial_{x}^{3} v_{\varepsilon}$ arising from $(0.7)$ shows that up to a (not relabelled) subsequence, $w_{\varepsilon}$ converge weakly in $L^{2}((0, T) \times(-a, a))$ to some $w$ belonging to this space. We next work on identifying this function $w$. Since

$$
v_{\varepsilon} \underset{\varepsilon \rightarrow 0}{\longrightarrow} v \quad \text { pointwise uniformly in }[0, T] \times(-a, a)
$$

we get that

$$
v_{\varepsilon}^{n} \underset{\varepsilon \rightarrow 0}{\longrightarrow} v^{n} \quad \text { pointwise in }(0, T) \times(-a, a) .
$$

Thus, it suffices to show that

$$
f_{\varepsilon}\left(v_{\varepsilon}\right)-v_{\varepsilon}^{n} \underset{\varepsilon \rightarrow 0}{\longrightarrow} 0 \quad \text { pointwise in }(0, T) \times(-a, a)
$$

in order to infer

$$
\left[f_{\varepsilon}\left(v_{\varepsilon}\right)\right]^{1 / 2} \underset{\varepsilon \rightarrow 0}{\longrightarrow} v^{n / 2} \quad \text { pointwise }
$$


But

$$
f_{\varepsilon}\left(v_{\varepsilon}\right)=\frac{v_{\varepsilon}^{4} f\left(v_{\varepsilon}\right)}{\varepsilon f\left(v_{\varepsilon}\right)+v_{\varepsilon}^{4}}, \quad f\left(v_{\varepsilon}\right)=v_{\varepsilon}^{n}, \quad v_{\varepsilon}>0
$$

SO

$$
\left|f_{\varepsilon}\left(v_{\varepsilon}\right)-v_{\varepsilon}^{n}\right|=\left|\frac{\varepsilon v_{\varepsilon}^{2 n}}{\varepsilon v_{\varepsilon}^{n}+v_{\varepsilon}^{4}}\right| .
$$

On the set $\{v>0\}$, due to the uniform convergence of $v_{\varepsilon}$ to $v$, we have

$$
v_{\varepsilon}(t, x)>\frac{v(t, x)}{2}>0 \quad \text { for } \varepsilon \text { sufficiently small, }
$$

which implies

$$
\left|f_{\varepsilon}\left(v_{\varepsilon}\right)-v_{\varepsilon}^{n}\right|=\left|\frac{\varepsilon}{\varepsilon v_{\varepsilon}^{-n}+v_{\varepsilon}^{4-2 n}}\right| \leq \frac{\varepsilon}{v^{4-2 n}} \underset{\varepsilon \rightarrow 0}{\longrightarrow} 0 \text { for } 0<n<2 .
$$

On the set $\{v=0\}$, we have

$$
v_{\varepsilon}(t, x) \underset{\varepsilon \rightarrow 0}{\longrightarrow} 0 \quad \Longrightarrow \quad f\left(v_{\varepsilon}(t, x)\right) \underset{\varepsilon \rightarrow 0}{\longrightarrow} 0 .
$$

But $0 \leq f_{\varepsilon}\left(v_{\varepsilon}\right) \leq f\left(v_{\varepsilon}\right)$ implies

$$
f_{\varepsilon}\left(v_{\varepsilon}\right) \underset{\varepsilon \rightarrow 0}{\longrightarrow} 0
$$

Clearly, from (2.57) and (2.58) we have

$$
f_{\varepsilon}\left(v_{\varepsilon}\right) \underset{\varepsilon \rightarrow 0}{\longrightarrow} v^{n} \quad \Longrightarrow \quad\left[f_{\varepsilon}\left(v_{\varepsilon}\right)\right]^{1 / 2} \underset{\varepsilon \rightarrow 0}{\longrightarrow} v^{n / 2} \quad \text { pointwise. }
$$

We have $f_{\varepsilon}\left(v_{\varepsilon}\right) \leq M^{n}$, where $M$ is the upper bound for $v_{\varepsilon}$ for all $0<\varepsilon<1$. So, by Dominated Convergence Theorem we have

$$
\left[f_{\varepsilon}\left(v_{\varepsilon}\right)\right]^{1 / 2} \underset{\varepsilon \rightarrow 0}{\longrightarrow} v^{n / 2} \quad \text { strongly in } L^{2}((0, T) \times(-a, a)) .
$$

In fact, $\left[f_{\varepsilon}\left(v_{\varepsilon}\right)\right]^{1 / 2}$ converges strongly to $v^{n / 2}$ in any $L^{p}((0, T) \times(-a, a))$ for $1 \leq p<\infty$. Since we have

$$
w_{\varepsilon} \underset{\varepsilon \rightarrow 0}{\rightarrow} w \quad \text { weakly in } L^{2}((0, T) \times(-a, a)) \text {, }
$$


(2.59) implies

$$
\left[f_{\varepsilon}\left(v_{\varepsilon}\right)\right]^{1 / 2} w_{\varepsilon} \underset{\varepsilon \rightarrow 0}{\rightarrow} v^{n / 2} w \quad \text { weakly in } L^{1}((0, T) \times(-a, a)) .
$$

Substitute $w_{\varepsilon}=\left[f_{\varepsilon}\left(v_{\varepsilon}\right)\right]^{1 / 2} \partial_{x}^{3} v_{\varepsilon}$ to get

$$
f_{\varepsilon}\left(v_{\varepsilon}\right) \partial_{x}^{3} v_{\varepsilon} \underset{\varepsilon \rightarrow 0}{\longrightarrow} v^{n / 2} w \quad \text { in } \quad L^{1}((0, T) \times(-a, a)) .
$$

According to Lemma 4.9 [10], $f_{\varepsilon}\left(v_{\varepsilon}\right) \partial_{x}^{3} v_{\varepsilon}$ converges strongly in $L^{2}(\Omega)$ to $v^{n} \partial_{x}^{3} v \chi_{\mathcal{R}_{T} \cap \Omega}$ for all $\Omega \Subset(0, T) \times(-a, a)$. We conclude that $w=v^{n / 2} \partial_{x}^{3} v \chi_{\mathcal{R}_{T}}$ a.e. in $(0, T) \times(-a, a)$. Thus, the obvious

$$
J\left[v_{\varepsilon}(t, \cdot)\right]+\int_{s}^{t} \int_{-a}^{a} f_{\varepsilon}\left(v_{\varepsilon}(\tau, x)\right) \partial_{x}^{3} v_{\varepsilon}^{2}(\tau, x) d x d \tau=J\left[v_{\varepsilon}(s, \cdot)\right] \quad \text { for all } \quad 0 \leq s \leq t \leq T
$$

implies (2.47) for $u_{k}$.

To pass from $u_{k}$ to $u$, we note that we have the same available estimates as above. We have already seen that $u_{k}$ converges to $u$ in $L^{2}\left(0, T ; H^{1}(\mathbb{R})\right)$. Also, we have seen that $u_{k}$ converges uniformly on compact sets to $u$. This, together with the uniform bound of $u_{k}^{n / 2} \partial_{x}^{3} u_{k} \chi_{\mathcal{R}_{k, T}}$ in $L^{2}((0, T) \times \mathbb{R})$, implies $u_{k}^{n / 2} \partial_{x}^{3} u_{k} \chi_{\mathcal{R}_{k, T}}$ converges weakly in $L^{2}((0, T) \times \mathbb{R})$ to $u^{n / 2} \partial_{x}^{3} u \chi_{\mathcal{R}_{T}}$, since Lemma $4.9[10]$ applies in this case as well. Indeed, a careful inspection of the proof shows that we only need the uniform convergence of $u_{k}^{n / 2} \partial_{x}^{3} u_{k}$ to $u^{n / 2} \partial_{x}^{3} u$ in the set $\{u \geq \delta\}$ for any $\delta>0$. This easily follows, however, from the uniform parabolicity of the problem in that set. Thus, (2.45) holds for $u$ as well.

\subsection{Existence result for a relaxed problem}

Let us now consider the effect of a second-order "porous-media" term on the evolution of weak solutions of the fourth-order degenerate diffusion equation.

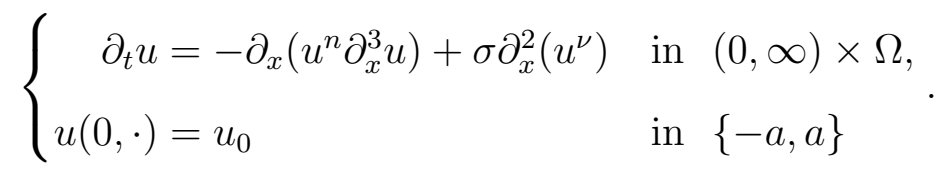


for some $\sigma>0$ and $1<\nu<2$.

This problem is considered by Tudorascu in [42] on a bounded interval for $\sigma>0,1 / 2<$ $\nu<2$, and by Bertozzi and Pugh in [9] when $\sigma=1,1<\nu<2$, the addition of the second-order term being justified by its behavior as a cut-off of Van der Waals interaction. Note that if $\sigma=0$, then we are, obviously, back to (2.38).

Proposition 2.14. (Global existence of unique smooth positive solutions for the regularized relaxed problem [10]):

Let $u_{0} \in H^{1}(\Omega), u_{0} \geq 0,1<\nu<2$. Given an initial condition

$$
u_{0 \varepsilon}=u_{0}+\varepsilon^{\theta}
$$

there exists a unique positive solution $u_{\varepsilon}$ to the regularized equation

$$
\partial_{t} u_{\varepsilon}=-\partial_{x}\left(f_{\varepsilon}\left(u_{\varepsilon}\right) \partial_{x}^{3} u_{\varepsilon}\right)+\sigma \partial_{x}^{2} u_{\varepsilon}^{\nu}
$$

with no flux boundary conditions at $(0, \infty) \times\{-a, a\}$, where $f_{\varepsilon}$ is the regularizing function $(2.33)$.

The proof is a minor modification of that presented above for Proposition (2.6).

Proposition 2.15. (Existence of Weak Solution for the relaxed problem (2.60) [9] if $1<$ $\nu<2$ and [42] if $1 / 2<\nu<2$ )

Consider $0<n<\infty, 1 / 2<\nu<2$ and a probablity density $u_{0} \in H^{1}(\Omega)$. Let $u_{\varepsilon}$ be the unique solution of (2.61) with no-flux boundary conditions and

$$
u_{0 \varepsilon}=u_{0}+\varepsilon^{\theta}
$$

for some $0<\theta<2 / 5$. Then there exists a subsequence (not relabeled) $\left\{u_{\varepsilon}\right\}_{\varepsilon \downarrow 0}$ which converges pointwise uniformly and weakly in $L^{2}\left((0, T) ; H^{2}(\Omega)\right)$ and $L^{\infty}\left((0, T) ; H^{1}(\Omega)\right)$ to some

$$
u \in L^{2}\left((0, T) ; H^{2}(\Omega)\right) \cap L^{\infty}\left((0, T) ; H^{1}(\Omega)\right) .
$$


Moreover, $u$ is a solution for (2.60) in the sense

$$
\iint_{Q_{T}} u \partial_{t} \varphi d x d t=-\iint_{\mathcal{R}} u^{n} \partial_{x}^{3} u \partial_{x} \varphi d x d t+\sigma \iint_{Q_{T}} \partial_{x} u^{\nu} \partial_{x} \varphi d x d t
$$

This result is proved in [10] for the case $\sigma=1,1<\nu<2$ and in [42] it was showed that it is, in fact, valid for all $\sigma>0$ and $1 / 2<\nu<2$. 


\section{Chapter 3}

\section{Asymptotic decay}

\subsection{Asymptotic decay for a thin film equation in a bounded domain}

Entropy methods are used to establish various proofs of convergence of solutions to the thin film equation to the mean value (steady state). Numerous results on this topic exist in the literature. Below, a selection of results given in [5], [12], [33], [42] is presented.

Proposition 3.1. [5] Let $0<n<3,0 \leq u_{0} \in H^{1}(\Omega)$ and $u_{0} \not \equiv 0 \in \Omega$, then the weak solution $u$ constructed in (2.7) converges to its mean value as $t \rightarrow \infty$ :

$$
u(t, x) \rightarrow \frac{1}{|\Omega|} \int_{\Omega} u_{0}(x) d x
$$

uniformly in $\Omega$ as $t \rightarrow \infty$.

In [33] the authors investigated the large-time behavior of weak solutions to (2.1). In particular, for all $n>0$, they proved exponential decay of $u(t, x)$ towards its mean value $\frac{1}{|\Omega|} \int_{\Omega} u_{0}(x) d x$ in $L^{1}$-norm for long time and they gave the explicit ( $n$-dependent) rate of decay. Their result is based on classical entropy estimates, and on detailed lower bounds for the entropy production. [12] proved a lower bound on the rate of relaxation to equilibrium in the $H^{1}$-norm, this result is a first in that gives decay in the $H^{1}$-norm, an obvious improvement to $L^{1}$ or even $L^{\infty}$ results [5], [10], [13], [33]. [42] proved exponential asymptotic decay in the $H^{1}$-norm for nonnegative weak solutions of (2.1). 


\subsection{Asymptotic decay for the Cauchy problem}

The optimal rates of decay from [6] can now be translated to these not necessarily compactly supported dissipative strong solutions.

Proposition 3.2. Assume that $3 / 8<n<2$ and $u$ is the dissipative strong solution for (2.38) constructed above corresponding to an initial nonnegative $u_{0} \in L^{1}(\mathbb{R}) \cap H^{1}(\mathbb{R})$ such that $H\left[u_{0}\right]<\infty$. Then there exists a constant $C>0$ depending only on $J\left[u_{0}\right]$ and $n$ such that

$$
J[u(t, \cdot)] \leq C t^{-3 /(n+4)} \quad \text { for all } t>0 .
$$

\subsubsection{Our result for the Cauchy problem}

Throughout this entire section we deal with mild solutions in the spirit of Definition 2.11. Due to the continuity of $u(t, \cdot)$ for any $t \geq 0$, we have that $\mathcal{P}_{t}$ is an open set. Thus,

$$
\mathcal{P}_{t}=\bigcup_{j=1}^{m_{t}}\left(a_{j}, b_{j}\right)
$$

where the intervals $\left(a_{j}, b_{j}\right)$ are mutually disjoint. They are also bounded, except possibly for two of them. Also, $m_{t}$ may be equal to infinity. While our result has no bearing on the important question of decay to self-similarity in the $L^{1}$-norm, it does generalize the findings of Carlen and Ulusoy [11] in two different directions. First, we carry out our analysis for the case of dissipative mild solutions, while the analysis in [11] is only formal (as existence of classical solutions is still open). Secondly, we deal with an entire array of PDE's of the type (2.38) for $0<n<2$, as opposed to [11] where the case $n=1$ alone is analyzed.

Proposition 3.3. Assume that $0<n \leq 2$ and $u$ is a dissipative mild solution for (2.38) corresponding to an initial probability density $u_{0} \in H^{1}(\mathbb{R})$. The following are true:

(i) If $0<n \leq 1$, then there exists a constant $C>0$ depending only on $J\left[u_{0}\right]$ and $n$ such that

$$
\int_{\mathcal{P}_{t}} u^{n}(t, x)\left(\partial_{x}^{3} u(t, x)\right)^{2} d x \geq C J^{3}[u(t, \cdot)] \quad \text { for all } t>0
$$

(ii) If $1<n \leq 2$ and $\operatorname{spt}[u(t, \cdot)]$ is bounded for all $t \geq 0$, then (3.3) holds. 
First, let us prove the following lemma, which is the key ingredient in the proofs of Proposition 3.3 and Theorem 3.7.

Lemma 3.4. Assume that $u$ is a dissipative mild solution for (2.38) corresponding to an initial probability density $u_{0} \in H^{1}(\mathbb{R})$. Then

(i) For $0<n \leq 1$, the function $\left(a_{j}, b_{j}\right) \ni x \rightarrow u(t, x) \partial_{x}^{2} u(t, x)$ is bounded for almost all $t>0$ and all $j=1, \ldots, m_{t}$.

(ii) For $1<n \leq 2$, if $\operatorname{spt}[u(t, \cdot)]$ is bounded for all $t \geq 0$, then the function $\left(a_{j}, b_{j}\right) \ni x \rightarrow$ $u(t, x) \partial_{x}^{2} u(t, x)$ is bounded for almost all $t>0$ and all $j=1, \ldots, m_{t}$.

Proof. (i) By (0.6) we deduce that

$$
\int_{\mathcal{P}_{t}} u^{n}(t, x)\left(\partial_{x}^{3} u(t, x)\right)^{2} d x<\infty \quad \text { for a.e. } t>0
$$

We denote by $\mathcal{T}$ the set of all $t>0$ for which the above integrability holds true and for which $u(t, \cdot) \in H^{2}(\mathbb{R})$. First assume $\left(a_{j}, b_{j}\right)$ is bounded. Since, according to [11], we have

$$
\|u\|_{L^{\infty}((0, \infty) \times \mathbb{R})} \leq 1+\sqrt{2} J\left[u_{0}\right]=: M
$$

the Cauchy-Schwarz inequality implies

$$
\begin{aligned}
M^{1-n} \int_{\mathcal{P}_{t}} u^{n}(t, x)\left(\partial_{x}^{3} u(t, x)\right)^{2} d x & \geq \int_{a_{j}}^{b_{j}} u^{2-n}(t, x) d x \int_{\mathcal{P}_{t}} u^{n}(t, x)\left(\partial_{x}^{3} u(t, x)\right)^{2} d x \\
& \geq\left(\int_{z}^{x} u(t, y) \partial_{x}^{3} u(t, y) d y\right)^{2}
\end{aligned}
$$

for any $z, x \in\left(a_{j}, b_{j}\right)$. Thus,

$$
\left|u(t, x) \partial_{x}^{2} u(t, x)-u(t, z) \partial_{x}^{2} u(t, z)-\int_{z}^{x} \partial_{x} u(t, y) \partial_{x}^{2} u(t, y) d y\right| \leq C=C(t)<\infty
$$

for all $t \in \mathcal{T}$. Since $\partial_{x} u\left(t, a_{j}\right)=0=\partial_{x} u\left(t, b_{j}\right)$ and $\partial_{x} u(t, \cdot)$ is continuous on $\left[a_{j}, b_{j}\right]$ and differentiable on $\left(a_{j}, b_{j}\right)$, Rolle's theorem implies there exists $x_{0} \in\left(a_{j}, b_{j}\right)$ such that $\partial_{x}^{2} u\left(x_{0}, t\right)=0$. 
Thus, the last displayed inequality applied to $z=x_{0}$ gives

$$
\left|u(t, x) \partial_{x}^{2} u(t, x)\right| \leq C+\frac{1}{2}\left[\left(\partial_{x} u\left(t, x_{0}\right)\right)^{2}+\left(\partial_{x} u(t, x)\right)^{2}\right]
$$

Since $\partial_{x} u(t, \cdot) \in L^{\infty}(\mathbb{R})$, the proof is concluded if $\left(a_{j}, b_{j}\right)$ is bounded. Otherwise, either $\mathcal{P}_{t}=\mathbb{R}$ or, without loss of generality, we may assume $\left(a_{j}, b_{j}\right)=(a, \infty)$ for some $a \in \mathbb{R}$. Let us treat the latter case, as the former is almost identical. Since

$$
u(t, a)=0=\lim _{x \rightarrow \infty} u(t, x) \text { and } \quad u(t, \cdot)>0 \quad \text { on } \quad(a, \infty)
$$

we infer that there exist at least two distinct points $y \in(a, \infty)$ such that $\partial_{x}^{2} u(t, y)=0$. Then we finish the argument as above.

(ii) For $1<n \leq 2, \int_{a_{j}}^{b_{j}} u^{2-n}(t, x) d x$ is bounded independently of the length of $\left(a_{j}, b_{j}\right)$. Indeed, if $u(t, \cdot)$ is compactly supported, then

$$
\int_{a_{j}}^{b_{j}} u^{2-n}(t, x) d x \leq M^{2-n} \mathcal{L}^{1}(\operatorname{spt}[u(t, \cdot)])
$$

where $M$ is the upper bound for $u$ [11]. Then, we finish the argument as in case (i).

Remark 3.5. If $1<n \leq 2$, then we are using an extra assumption namely the support of $u(t, \cdot)$ is bounded for all $t>0$.

Proof of Proposition 3.3. Let $t>0$ be arbitrarily fixed. We may also assume $t \in \mathcal{T}$, otherwise the left hand side of (3.3) is $\infty$ and the inequality will automatically be satisfied. Indeed, (0.6) shows $J[u(t, \cdot)] \leq J\left[u_{0}\right]<\infty$ for all $t \geq 0$. The Cauchy-Schwarz inequality yields

$$
\int_{\mathbb{R}} u^{2-n}(t, x)\left(\partial_{x} u(t, x)\right)^{2} d x \int_{\mathcal{P}_{t}} u^{n}(t, x)\left(\partial_{x}^{3} u(t, x)\right)^{2} d x \geq\left(\int_{\mathcal{P}_{t}} u(t, x) \partial_{x} u(t, x) \partial_{x}^{3} u(t, x) d x\right)^{2} .
$$


We have

$$
\begin{aligned}
\int_{\mathcal{P}_{t}} u(t, x) \partial_{x} u(t, x) \partial_{x}^{3} u(t, x) d x & =\sum_{j=1}^{m_{t}} \int_{a_{j}}^{b_{j}} u(t, x) \partial_{x} u(t, x) \partial_{x}^{3} u(t, x) d x \\
& =\left.\sum_{j=1}^{m_{t}} u(t, x) \partial_{x} u(t, x) \partial_{x}^{2} u(t, x)\right|_{a_{j}} ^{b_{j}}-\sum_{j=1}^{m_{t}} \int_{a_{j}}^{b_{j}}\left(\partial_{x} u(t, x)\right)^{2} \partial_{x}^{2} u(t, x) d x \\
& -\sum_{j=1}^{m_{t}} \int_{a_{j}}^{b_{j}} u(t, x)\left(\partial_{x}^{2} u(t, x)\right)^{2} d x
\end{aligned}
$$

Since $\partial_{x} u\left(t, a_{j}\right)=0=\partial_{x} u\left(t, b_{j}\right)$ and $\partial_{x} u(t, x) \rightarrow 0$ as $|x| \rightarrow \infty$, we deduce, in light of Lemma 3.4, that the boundary terms vanish. Also, the second term in the second row of the above display integrates to zero. Therefore,

$$
\int_{\mathcal{P}_{t}} u(t, x) \partial_{x} u(t, x) \partial_{x}^{3} u(t, x) d x=-\int_{\mathcal{P}_{t}} u(t, x)\left(\partial_{x}^{2} u(t, x)\right)^{2} d x=-\int_{\mathbb{R}} u(t, x)\left(\partial_{x}^{2} u(t, x)\right)^{2} d x
$$

The fact that $M \geq u(t, x) \geq 0,(3.4)$ and (6.18) yield

$$
2 M^{2-n} J[u(t, \cdot)] \int_{\mathcal{P}_{t}} u^{n}(t, x)\left(\partial_{x}^{3} u(t, x)\right)^{2} d x \geq\left(\int_{\mathbb{R}} u(t, x)\left(\partial_{x}^{2} u(t, x)\right)^{2} d x\right)^{2} .
$$

By Cauchy-Schwarz

$$
\int_{\mathbb{R}} u(t, x) d x \int_{\mathbb{R}} u(t, x)\left(\partial_{x}^{2} u(t, x)\right)^{2} d x \geq\left(\int_{\mathbb{R}} u(t, x) \partial_{x}^{2} u(t, x) d x\right)^{2},
$$

which, in view of $u(t, \cdot)$ being a probability density, gives ( integrating by parts in the right hand side and using that $u(t, \cdot)$ and $\partial_{x} u(t, \cdot)$ vanish at $\left.\pm \infty\right)$

$$
\int_{\mathbb{R}} u(t, x)\left(\partial_{x}^{2} u(t, x)\right)^{2} d x \geq 4 J^{2}[u(t, \cdot)]
$$

In light of (3.6), the above inequality yields (3.3) with $C:=8 M^{n-2}$ for $0<n<2$, and with $C:=8$ for $n=2$ (and thus independent of $u_{0}$ ).

Remark 3.6. The proofs work directly applied to $u$ instead of $u_{k}$ for the case (i) of the Theorem 3.7 and Corollary 3.8. 
Theorem 3.7. Assume that $u$ is a dissipative mild solution for (2.38) corresponding to an initial probability density $u_{0} \in H^{1}(\mathbb{R})$. The following are true:

(i) For $0<n \leq 1$, there exists a finite $0<\lambda$ such that

$$
J[u(t, \cdot)] \leq \frac{\lambda}{\sqrt{t+1}} \quad \text { for all } t \geq 0
$$

(ii) For $1<n \leq 2$, let $u$ be the limit of compactly supported dissipative mild solutions constructed in the proof of Theorem 2.13. Then (3.7) holds.

Proof. (ii) First we show that it holds for the approximations $u_{k}$ and then we pass to the limit to get it for $u$. In light of (2.45) and (3.3), we may write

$$
J\left[u_{k}(t, \cdot)\right]+C \int_{s}^{t} J^{3}\left[u_{k}(\tau, \cdot)\right] d \tau \leq J\left[u_{k}(s, \cdot)\right] \quad \text { for almost all } t \geq s \geq 0
$$

According to $(3.8), J\left[u_{k}(t, \cdot)\right]$ coincides a.e. with a nonincreasing positive function $\varphi(t)$ whose a.e. pointwise derivative satisfies,

$$
\dot{\varphi}(t) \leq-C \varphi^{3}(t) \quad \text { for a.e. } t>0
$$

Set now $\phi(t):=\varphi^{-2}(t)$ and note that this is a nondecreasing positive function whose pointwise a.e. derivative satisfies

$$
\dot{\phi}(t) \geq 2 C \quad \text { for a.e. } t>0 \text {. }
$$

It follows by Corollary (6.2.4) in [38]

$$
\phi(t)-\phi(0)=\int_{0}^{t} d\|\dot{\phi}\|(s) \geq \int_{0}^{t} \dot{\phi}(s) d s \geq 2 C t \quad \text { for all } t>0
$$

So, $\phi(t) \geq \phi(0)+2 C t$, which implies

$$
\varphi^{-2}(t) \geq \varphi^{-2}(0)+2 C t \quad \text { for all } t>0
$$


Then,

$$
J^{-2}\left[u_{k}(t, \cdot)\right] \geq J^{-2}\left[u_{k 0}\right]+2 C t \quad \text { for all } t>0
$$

which implies

$$
J\left[u_{k}\right] \leq \frac{J\left[u_{k 0}\right]}{\sqrt{\left(2 C J^{2}\left[u_{k 0}\right]\right) t+1}} \quad \text { for all } t>0
$$

Thus,

$$
J\left[u_{k}\right] \leq \frac{\lambda_{k}}{\sqrt{t+1}} \quad \text { for all } t>0,
$$

where $\lambda_{k}=J\left[u_{k 0}\right] / \min \left\{1, \sqrt{2 C} J\left[u_{k 0}\right]\right\}$. We may pass to the limit as $k \rightarrow \infty$, since $u_{0 k} \rightarrow u_{0}$ in $\mathrm{E}^{1}(\mathbb{R}) \cap H^{1}(\mathbb{R})$ as $k \rightarrow \infty$, to get $J\left[u_{k 0}\right] \rightarrow J\left[u_{0}\right]$. It follows that $\lambda_{k} \rightarrow \lambda$, which implies (3.7) with $\lambda=J\left[u_{0}\right] / \min \left\{1, \sqrt{2 C} J\left[u_{0}\right]\right\}$. Here $\|\dot{\phi}\|$ denotes the total variation measure associated to $\phi$.

An easy consequence is the uniform asymptotic convergence of $u(t, \cdot)$ to 0 in the sup norm.

Corollary 3.8. Assume that $u$ is a dissipative mild solution for (2.38) corresponding to an initial probability density $u_{0} \in H^{1}(\mathbb{R})$. The following are true:

(i) For $0<n \leq 1$, there exists a finite $\Lambda>0$ such that

$$
\|u(t, \cdot)\|_{L^{\infty}(\mathbb{R})} \leq \Lambda(1+t)^{-1 / 8} .
$$

(ii) For $1<n \leq 2$, let $u$ be the limit of compactly supported dissipative mild solutions constructed in the proof of Theorem 2.13. Then (3.9) holds.

Proof. Since

$$
\left\|u_{k}(t, \cdot)\right\|_{L^{2}(\mathbb{R})}\left\|\partial_{x} u_{k}(t, \cdot)\right\|_{L^{2}(\mathbb{R})} \geq \int_{-\infty}^{x} u_{k}(t, y) \partial_{x} u_{k}(t, y) d y=\frac{1}{2} u_{k}^{2}(t, x)
$$

we immediately get (3.9) by using that $\left\|u_{k}(t, \cdot)\right\|_{L^{2}(\mathbb{R})}^{2} \leq M_{k}$ and passing to the limit as $k \rightarrow \infty$. The constant is $\Lambda=(8 \lambda M)^{1 / 4}$.

Note that convergence in the sup norm for the strong solution to the self-similar solution was proved in [13] for $n=1$. The rate obtained in [13] was $(t+1)^{-1 / 15}$. Here we show that 
this convergence holds for weak solutions with zero contact angle as well and is simply a consequence of the uniform decay to zero; we also improve the rate to $(t+1)^{-1 / 8}$ while at the same time we show that it holds true for all $0<n<2$. Of course, the main purpose of [13] was proving decay of strong solutions to the self-similar profiles which known to exist for $0<n<3$. The important aspect here is that the only relevant norm for this decay is the $L^{1}$-norm. Indeed, we show in this work that convergence to self-similarity in any $L^{p}$ for $1<p \leq \infty$ is merely a manifestation of the decay to zero of the film thickness.

\subsection{Our result for the relaxed problem}

If we consider a positive classical solutions to (2.60), then

$$
\frac{1}{2} \frac{d}{d t} \int_{\mathbb{R}}\left(\partial_{x} u\right)^{2} d x=-\int_{\mathbb{R}} u^{n}\left(\partial_{x}^{3} u\right)^{2} d x-\sigma \nu \int_{\mathbb{R}} u^{\nu-1}\left(\partial_{x}^{2} u\right)^{2} d x+\sigma \frac{\nu(\nu-1)(\nu-2)}{3} \int_{\mathbb{R}} u^{\nu-3}\left(\partial_{x} u\right)^{4} d x .
$$

Clearly, $J$ is a Lyapunov functional for non-negative smooth solution of (2.60) provided $\nu \in(1,2]$. Indeed, if $1 \leq \nu<2$, then (3.10) may be rewritten as

$$
\begin{aligned}
\frac{1}{2} \frac{d}{d t} \int_{\mathbb{R}}\left(\partial_{x} u\right)^{2} d x= & -\int_{\mathbb{R}} u^{n}\left(\partial_{x}^{3} u\right)^{2} d x-\nu \sigma \frac{2 \nu-1}{2-\nu} \int_{\mathbb{R}} u^{\nu-1}\left(\partial_{x}^{2} u\right)^{2} d x \\
& -\nu \sigma \frac{3(1-\nu)}{(2-\nu)}\left[\int_{\mathbb{R}} u^{\nu-1}\left(\partial_{x}^{2} u\right)^{2} d x-\frac{(2-\nu)^{2}}{9} \int_{\mathbb{R}} u^{\nu-3}\left(\partial_{x} u\right)^{4} d x\right] .
\end{aligned}
$$

We can apply Lemma 3 in [42] with $v=u(t, \cdot)$ and $\beta=\nu-1$ to conclude

$$
\int_{\mathbb{R}} u^{\nu-1}\left(\partial_{x}^{2} u\right)^{2} d x \geq \frac{(2-\nu)^{2}}{9} \int_{\mathbb{R}} u^{\nu-3}\left(\partial_{x} u\right)^{4} d x .
$$

Thus, in view of (3.11), the right hand side of (3.10) is still negative even if $\nu \in[1 / 2,1]$.

Remark 3.9. Before estimating the new terms appearing in the right hand side of (3.10) as a consequence of the perturbation, let us check whether the mass of $u^{3-\nu}$ is dissipating for 
$\nu \leq 2$

$$
\begin{aligned}
\frac{d}{d t} \int_{\mathbb{R}} u^{3-\nu} d x & =(3-\nu) \int_{\mathbb{R}} u^{2-\nu} \partial_{t} u d x \\
& =(3-\nu) \int_{\mathbb{R}} u^{2-\nu}\left[-\partial_{x}\left(u^{n} \partial_{x}^{3} u\right)+\sigma \partial_{x}^{2} u^{\nu}\right] d x \\
& =-(3-\nu) \int_{\mathbb{R}} u^{2-\nu} \partial_{x}\left(u^{n} \partial_{x}^{3} u\right) d x+\sigma(3-\nu) \int_{\mathbb{R}} u^{2-\nu} \partial_{x}^{2} u^{\nu} d x
\end{aligned}
$$

After integrating by parts twice and taking into account that $\partial_{x} u(t, x) \rightarrow 0$ as $|x| \rightarrow \infty$,

$$
\begin{array}{r}
\frac{d}{d t} \int_{\mathbb{R}} u^{3-\nu} d x=-(2-\nu)(3-\nu)\left[(n-\nu+1) \int_{\mathbb{R}} u^{n-\nu}\left(\partial_{x} u\right)^{2} \partial_{x}^{2} u d x+\int_{\mathbb{R}} u^{n-\nu+1}\left(\partial_{x}^{2} u\right)^{2} d x\right. \\
\left.+\nu \sigma \int_{\mathbb{R}}\left(\partial_{x} u\right)^{2} d x\right],
\end{array}
$$

which implies

$$
\frac{d}{d t} \int_{\mathbb{R}} u^{3-\nu} d x \leq 0
$$

Proposition 3.10. Assume that $0<n \leq 2,1 \leq \nu \leq 2$ and $u$ is a dissipative mild solution for (2.60) corresponding to an initial probability density $u \in H^{1}(\mathbb{R})$. Then the following are true:

(i) If $0<n \leq 1$, then there exist constants $C$ and $C_{\sigma, \nu}$ depending only on $J\left[u_{0}\right], n, \sigma$ and $\nu$ such that

$$
\int_{\mathcal{P}_{t}} u^{n}\left(\partial_{x}^{3} u\right)^{2} d x+\nu \sigma \frac{2 \nu-1}{2-\nu} \int_{\mathbb{R}} u^{\nu-1}\left(\partial_{x}^{2} u\right)^{2} d x \geq C J^{3}[u(t, \cdot)]+C_{\sigma, \nu} J^{2}[u(t, \cdot)] \text { for all } t>0 .
$$

(ii) If $1<n \leq 2$, and $\operatorname{spt}[u(t, \cdot)]$ is bounded for all $t \geq 0$, then (3.12) holds.

Proof. (i) For the first term of (3.12) we already know (from Proposition 3.3) that is bounded from below by $C J^{3}[u(t, \cdot)]$. For the second term, the Cauchy-Schwarz inequality implies

$$
\int_{\mathbb{R}} u^{3-\nu} d x \int_{\mathbb{R}} u^{\nu-1}\left(\partial_{x}^{2} u\right)^{2} d x \geq\left(\int_{\mathbb{R}} u \partial_{x}^{2} u d x\right)^{2}
$$

which leads to

$$
\int_{\mathbb{R}} u^{3-\nu} d x \int_{\mathbb{R}} u^{\nu-1}\left(\partial_{x}^{2} u\right)^{2} d x \geq 4 J^{2}[u(t, \cdot)]
$$


Then, we can use the uniform upper bound $M$ on $u$ to obtain

$$
\int_{\mathbb{R}} u^{\nu-1}\left(\partial_{x}^{2} u\right)^{2} d x \geq 4 M^{\nu-2} J^{2}[u(t, \cdot)]
$$

The above inequality and (3.3) yield (3.12) with $C$ being the constant defined in Proposition 3.3 , and $C_{\sigma, \nu}:=4 \sigma M^{\nu-2} \nu(2 \nu-1) /(2-\nu)$.

Theorem 3.11. Assume that $u$ is a dissipative mild solution for (2.60) corresponding to an initial probability density $u_{0} \in H^{1}(\mathbb{R})$ and $1 \leq \nu \leq 2$. For $\lambda>0$, let $\phi^{-1}(\cdot ; \lambda)$ be the inverse of the increasing bijective function $\phi(\cdot ; \lambda):[0, \infty) \rightarrow[-\lambda \log \lambda, \infty)$ given by $\phi(x ; \lambda):=x-\lambda \log (\lambda+x)$. The following are true:

(i) For $0<n \leq 1$, there exist constants $C, C_{\sigma, \nu}>0$ such that

$$
J[u(t, \cdot)] \leq \frac{C_{\sigma, \nu}}{\phi^{-1}\left(C_{\sigma, \nu}^{2} t+\phi\left(C_{\sigma, \nu} J^{-1}\left[u_{0}\right] ; C\right)\right)} \quad \text { for all } t \geq 0 .
$$

(ii) For $1<n \leq 2$, let $u$ be the limit of compactly supported dissipative mild solutions constructed in the proof of Theorem 2.13. Then (3.13) holds.

Proof. (i) (3.11) and (3.12) directly imply

$$
\frac{d}{d t} J[u(t, \cdot)] \leq-C J^{3}[u(t, \cdot)]-C_{\sigma, \nu} J^{2}[u(t, \cdot)]
$$

After integrating by partial fractions we get

$$
C \log \left(C+\frac{C_{\sigma, \nu}}{J[u(t, \cdot)]}\right)-C_{\sigma, \nu} \frac{1}{J[u(t, \cdot)]} \leq-C_{\sigma, \nu}^{2} t+C \log \left(C+\frac{C_{\sigma, \nu}}{J\left[u_{0}\right]}\right)-C_{\sigma, \nu} \frac{1}{J\left[u_{0}\right]}
$$

Set $y=1 / J[u(t, \cdot)]$ and $y_{0}=1 / J\left[u_{0}\right]$. Then we have

$$
-C \log \left(C+C_{\sigma, \nu} y\right)+C_{\sigma, \nu} y \geq C_{\sigma, \nu}^{2} t-C \log \left(C+C_{\sigma, \nu} y_{0}\right)+C_{\sigma, \nu} y_{0}
$$

Let $x:=C_{\sigma, \nu} y$ and $x_{0}:=C_{\sigma, \nu} y_{0}$. Then, (3.14) gives

$$
\phi(x ; C) \geq C_{\sigma, \nu}^{2} t+\phi\left(x_{0} ; C\right) .
$$


Since $\phi$ is increasing and $\phi^{\prime}(0 ; C)=0$, we have the inverse function $\phi^{-1}:[-C \log C, \infty) \rightarrow$ $[0, \infty)$. Thus,

$$
x \geq \phi^{-1}\left(C_{\sigma, \nu}^{2} t+\phi\left(x_{0} ; C\right)\right)
$$

which implies

$$
y \geq \frac{\phi^{-1}\left(C_{\sigma, \nu}^{2} t+\phi\left(C_{\sigma, \nu} y_{0} ; C\right)\right)}{C_{\sigma, \nu}},
$$

i.e.

$$
\frac{1}{J[u(t, \cdot)]} \geq \frac{\phi^{-1}\left(C_{\sigma, \nu}^{2} t+\phi\left(C_{\sigma, \nu} J^{-1}\left[u_{0}\right] ; C\right)\right)}{C_{\sigma, \nu}} .
$$

Therefore,

$$
J[u(t, \cdot)] \leq \frac{C_{\sigma, \nu}}{\phi^{-1}\left(C_{\sigma, \nu}^{2} t+\phi\left(C_{\sigma, \nu} J^{-1}\left[u_{0}\right] ; C\right)\right)}
$$

hence we have shown the desired thesis.

Observation 3.12. Note that when $\sigma=0$, the rate of decay of $J[u(t, \cdot)] \sim 1 / \sqrt{t+1}$ and if $\sigma>0$, then the rate of decay of $J[u(t, \cdot)] \sim 1 / t$.

Corollary 3.13. Assume that $u$ is a dissipative mild solution for (2.60) corresponding to an initial probability density $u_{0} \in H^{1}(\mathbb{R})$ and $1 \leq \nu \leq 2$. The following are true:

(i) For $0<n \leq 1$, there exists a finite $\Lambda_{\sigma, \nu}>0$ such that

$$
\|u(t, \cdot)\|_{L^{\infty}(\mathbb{R})} \leq \Lambda_{\sigma, \nu}\left(\phi^{-1}\left(C_{\sigma, \nu}^{2} t+\phi\left(C_{\sigma, \nu} J^{-1}\left[u_{0}\right] ; C\right)\right)\right)^{-1 / 4}
$$

(ii) For $1<n \leq 2$, let $u$ be the limit of compactly supported dissipative mild solutions constructed in the proof of Theorem 2.13. Then (3.15) holds.

Proof. The proof goes the same way as the proof of Corollary 3.8; we get (3.15) with a constant $\Lambda_{\sigma, \nu}=\left(8 M C_{\sigma, \nu}\right)^{1 / 4}$. 


\section{Part II}

Chemical reaction-diffusion networks; convergence of the method of lines 


\section{Chapter 4}

\section{Preliminaries}

\subsection{Chemical reaction networks}

The theory of chemical reaction networks $(\mathrm{CRN})$ is a general framework for modeling the development of chemical concentrations resulting from chemical reactions that occur simultaneously. This science has been developed by a group of scholars, including, Fritz Horn, Roy Jackson and Martin Feinberg in the early 1970's [25], [29], [30]. We can define a chemical reaction network as a set of chemical species (or reactants) which react to form a different set of species.

In other words, consider a set of $N$ chemical species with the vector of concentrations $c=$ $\left(c_{1}, \ldots, c_{N}\right)$, and $r$ reactions between these species. Reactions can be viewed formally as arrows between two complexes, which are formal linear combinations of the species. The reactions considered in this thesis are of the form

$$
A \underset{k_{1}}{\stackrel{k_{-1}}{\leftrightharpoons}} B
$$

and

$$
A+B \underset{k_{1}}{\stackrel{k_{-1}}{\leftrightarrows}} C
$$

where each reaction involves combinations of two or three species. In these models, $A, B, C$ represent chemical species and $A \rightarrow B, B \rightarrow A$ represent the reactions for the first model with complexes $A, B$ and $A+B \rightarrow C, C \rightarrow A+B$ represent the reactions for the second 
model, with complexes $A+B$ and $C$.

\subsection{Well-mixed chemical reaction networks}

In order to translate the chemical reaction network into a dynamic framework, we need to develop many assumptions, one of them being that the medium where reactions take place is well mixed, so there are no pockets of high concentrations of one type or another. These assumptions lead us to describing the reaction by a differential equation. In order to determine the dynamics, we need to determine the net stoichiometric change resulting from each reaction and the corresponding rate of the reaction. We will number the reactions from $1, \cdots, r$ and define $\vec{y}_{i}$ and $\vec{y}_{i}^{\prime}$ to be the vectors of stoichiometric coefficients of the reactant and product complex of the $i^{\text {th }}$ reaction, respectively. We can then represent the dynamics of the chemical reaction network as

$$
\frac{d \vec{c}}{d t}=\sum_{i=1}^{r}\left({\overrightarrow{y^{\prime}}}_{i}-\vec{y}_{i}\right) R_{i}(t, \vec{c}),
$$

where the rate of the $i^{t h}$ reaction is $R_{i}(t, \vec{c}) \geq 0$. Also, we can represent it in matrix form as

$$
\frac{d \vec{c}}{d t}=\Gamma \vec{R}(t, \vec{c})
$$

the stoichiometric matrix $\Gamma \in \mathbb{Z}^{N \times r}$ contains the reaction vectors ${\overrightarrow{y^{\prime}}}_{i}-\vec{y}_{i}, i=1, \cdots, r$, as its columns, and $\vec{R}(t, \vec{c})=\left[R_{1}(t, \vec{c}), \cdots, R_{r}(t, \vec{c})\right]^{T} \in \mathbb{R}_{\geq 0}^{r}$.

For the reaction $A+B \underset{k_{1}}{\stackrel{k_{-1}}{\leftrightarrows}} C$, we have only three species and two reactions. So the stoiciometric matrix $\Gamma=\left[\begin{array}{cc}-1 & 1 \\ -1 & 1 \\ 1 & -1\end{array}\right]_{3 \times 2}$.

\subsubsection{Law of mass action}

Mass action kinetic is the most commonly used form for the rate functions $R_{i}(t, \vec{c})$. By using mass action, we can assign to each reaction a positive rate constant $k_{i}>0, i=$ $1, \cdots, r$. The rate functions are then defined according to $R_{i}(t, \vec{c})=k_{i} c_{1}^{y_{i 1}} \cdots c_{n}^{y_{i n}}, i=1, \cdots, r$, 
where $\vec{c}^{y_{i}}=c_{1}^{y_{i 1}} \cdots c_{n}^{y_{i n}}$. Then we can write the system of ordinary differential equations corresponding to a chemical reaction network under mass-action kinetics as

$$
\frac{d \vec{c}}{d t}=\sum_{i=1}^{r} k_{i}\left(\vec{y}_{i}^{\prime}-\vec{y}_{i}\right) \vec{c}^{y_{i}}
$$

Now let us go back to the reactions considered in this thesis. If we have

$$
A \underset{k_{1}}{\stackrel{k_{-1}}{\leftrightarrows}} B
$$

then $A$ is transformed into $B$ with rate $k_{1}>0$ and $B$ is transformed back into $A$ with rate $k_{-1}$; let $a$ and $b$ represent the concentrations of $A$ and $B$ (often denoted by $a=[A], b=[B]$ ) respectively. Then the ODEs for $a$ and $b$ are:

$$
\begin{aligned}
& \frac{d a}{d t}=-k_{1} a+k_{-1} b, \\
& \frac{d b}{d t}=k_{1} a-k_{-1} b .
\end{aligned}
$$

If, on the other hand, we study

$$
A+B \underset{k_{1}}{\stackrel{k_{-1}}{\leftrightarrows}} C
$$

then $A$ and $B$ must combine to form $C$, so the rate of the forward reaction is proportional to the product of the concentrations $a$ and $b$, while the backward reaction is proportional to $c=[C]$. Thus, the ODEs become

$$
\begin{aligned}
& \frac{d a}{d t}=-k_{1} a b+k_{-1} c \\
& \frac{d b}{d t}=-k_{1} a b+k_{-1} c \\
& \frac{d c}{d t}=k_{1} a b-k_{-1} c .
\end{aligned}
$$

Note that (4.2) is a system of nonlinear equations, while (4.1) are linear. Thus, we can write (4.1) in matrix form as $\dot{\vec{u}}=\mathcal{A} \vec{u}$, where $\vec{u}=[a, b]^{T}$ and

$$
\mathcal{A}=\left[\begin{array}{cc}
-k_{1} & k_{-1} \\
k_{1} & -k_{-1}
\end{array}\right]
$$


For (4.2) we have

$$
\dot{\vec{u}}=f(\vec{u})
$$

where $\vec{u}=[a, b, c]^{T}$ and

$$
f(\vec{u})=\left[\begin{array}{c}
-k_{1} a b+k_{-1} c \\
-k_{1} a b+k_{-1} c \\
k_{1} a b-k_{-1} c
\end{array}\right] .
$$

Remark 4.1. If the chemical system is well-mixed (isotropic mixtures), then we can write the chemical reactions as the system of ordinary differential equations based on the mass action law (as we did above), but if the chemical system is not well-mixed (anisotropic mixtures), we have to take into account the fact that chemical concentrations may vary in space.

\subsection{Chemical reaction-diffusion networks (CRDN)}

If we consider, for simplicity, a small interval on the line inside which we have a concentration $u$ of some reacting species, then the diffusive influx $J_{i n}$ of $u$ into one side of the small region will depend on the concentration gradient, $\partial_{x} u$, at that boundary and the diffusion coefficient, $D$, as

$$
J_{i n}=-D\left(\partial_{x} u\right)_{i n}
$$

The parameter $D>0$ is called diffusivity. The diffusive outflux from the region at the other side $J_{\text {out }}$ will similarly be given by

$$
J_{\text {out }}=-D\left(\partial_{x} u\right)_{\text {out }},
$$

where the concentration gradient is now evaluated at the other boundary. The rate at which the concentration grows due to diffusion then depends on the rate of change of the difference between these two fluxes, and so it involves the second derivative $\partial_{x}^{2} u$. If we add a kinetic reaction rate term $f(u)$, then the reaction-diffusion equation, which gives the rate of change of the concentration $u$ in time at any spatial point, has the general form

$$
\partial_{t} u=D \partial_{x}^{2} u+f(u)
$$


From a qualitative point of view, a reaction-diffusion system is a mathematical model describing how the concentrations of a number of substances vary over time and space under the influence of two terms: (1) reaction term or source term, in which concentration is generated or degenerated by local interaction, (2) diffusion term which causes the substances to spread out in space. In particular, a reaction-diffusion equation comprises a reaction term and a diffusion term, i.e. the typical form is as follows:

$$
\partial_{t} u=D \partial_{x}^{2} u+f(u)
$$

where $u=u(t, x)$ is a state variable and describes the density/concentration of a substance or a population of species at a time $t$ and a position $x \in \Omega \subset \mathbb{R}$ ( $\Omega$ is an open set) and $\partial_{x}^{2}$ denotes the Laplace operator. So the first term on the right hand side describes the diffusion ( $D$ is the diffusion coefficient). The second term, $f(u)$, describes the change of $u$, i.e. something happens to it (birth, death, chemical reaction, etc), besides spatial diffusion.

\subsection{The method of lines (Semi-discretizations)}

The method of lines (MOL) is a technique for solving partial differential equations in which all but one dimension are discretized. The method of lines most often refers to the construction or analysis of numerical methods for partial differential equations that proceeds by first discretizing the spatial derivatives only and leaving the time variable that continuous. This leads to a system of ordinary differential equations. The assumption is the ordinary differential equations are easier to analyze and solve than the partial differential equations. The approximation can be based on finite differences, finite elements. A method of lines obtained with finite differences seems easiest to apply and will be used exclusively in this thesis. The method of lines with continuous time is sometimes called the vertical method of lines.

In other words, the method of lines refers to an approximation of one or more partial differential equations with ordinary differential equations in just one of the independent variables. 


\subsubsection{Method of lines for chemical reaction-diffusion networks}

As we mentioned above, the method of lines (MOL) is a general way of viewing a partial differential equation as a system of ordinary differential equations.

The basic idea behind the MOL methodology is straightforward, and thus a simple example illustrates better than mere words the fundamental idea of the method.

Example: Consider a classical one-dimensional heat equation (diffusion equation):

$$
\left\{\begin{array}{cc}
\partial_{t} u=\partial_{x}^{2} u & t>0, x \in(0,1), \\
u(0, \cdot)=u_{0}(x), & x \in(0,1), \\
\partial_{t} u(t, 0)=\partial_{t} u(t, 1)=0 . &
\end{array}\right.
$$

Choose $N \geq 2$. Take a uniform grid $x_{k}$ with spacing $h=\frac{1}{N}$ such that $x_{k}=k h$ and $k=1, \cdots, N$.

Let $u_{k}(t)$ be the value of $u\left(t, x_{k}\right)$ and

$$
\partial_{x}^{2} u \approx \frac{1}{h^{2}}\left(u_{k-1}^{N}-2 u_{k}^{N}+u_{k+1}^{N}\right)
$$

By inserting the approximation for the second derivative in Eq.(4.4) we obtain, approximately:

$$
\partial_{t} u=\frac{1}{h^{2}}\left(u_{k-1}^{N}-2 u_{k}^{N}+u_{k+1}^{N}\right)
$$

Next, we need to discuss what is to be done with the boundary conditions. They are handled in the following way:

For the left end point $x=0(k=0)$ we use the forward difference approximation

$$
\left.\partial_{x} u(t)\right|_{x=0} \approx \frac{u_{1}^{N}(t)-u_{0}^{N}(t)}{h}=0 .
$$

At $k=0$ we have $u_{0}^{N}(t)=u_{1}^{N}(t)$. For the right end point $x=1$ at $k=N$ we use the backward difference approximation:

$$
\left.\partial_{x} u(t)\right|_{x=1} \approx \frac{u_{N}^{N}(t)-u_{N+1}^{N}(t)}{h}=0
$$


which gives $u_{N+1}^{N}(t)=u_{N}^{N}(t)$. The PDE now becomes a system of ODEs.

Introduction of the vector

$$
\vec{u}^{N}(t)=\left[u_{1}^{N}(t), \ldots, u_{N}^{N}(t)\right]^{T} \in \mathbb{R}^{N}
$$

allows us to write of ODEs in matrix form:

$$
\partial_{t} \vec{u}^{N}(t)=\Delta^{N} \vec{u}^{N}(t)
$$

where

$$
\Delta^{N}:=N^{2}\left[\begin{array}{ccccccc}
-1 & 1 & 0 & 0 & 0 & \ldots & 0 \\
1 & -2 & 1 & 0 & 0 & \ldots & 0 \\
0 & 1 & -2 & 1 & 0 & \ldots & 0 \\
\vdots & \vdots & \vdots & \vdots & \vdots & \vdots & \vdots \\
0 & 0 & \ldots & 0 & 1 & -2 & 1 \\
0 & 0 & \ldots & 0 & 0 & 1 & -1
\end{array}\right]_{N \times N}
$$

is a discrete approximation of $\partial_{x}^{2}$. We have thus transformed the PDF into a large system of ODEs.

\subsubsection{The connection between MOL and CRDN}

The main motivation of this part is to prove convergence of a solution to the semi-discrete scheme (ODEs) to a solution of a chemical reaction-diffusion system (PDEs). We will start with a simple model

$$
A \underset{k_{1}}{\stackrel{k_{-1}}{\leftrightharpoons}} B
$$

Consider one-dimensional chemical reaction-diffusion system of two species with Neumann boundary condition. 


$$
\left\{\begin{array}{l}
\partial_{t} a(t, x)=-k_{1} a(t, x)+k_{-1} b(t, x)+k_{A} \partial_{x}^{2} a(t, x) . \\
\partial_{t} b(t, x)=k_{1} a(t, x)-k_{-1} b(t, x)+k_{B} \partial_{x}^{2} b(t, x) . \\
a(0, x)=a_{0}(x) \\
b(0, x)=b_{0}(x) \\
\partial_{x} a(t, 0)=0 \text { and } \partial_{x} a(t, 1)=0 \\
\partial_{x} b(t, 0)=0 \text { and } \partial_{x} b(t, 1)=0
\end{array}\right.
$$

which is a system of linear parabolic partial differential equations. Let us now apply the method of lines to the system (5.1).

For $N \geq 2$, take $k=1, . ., N$ and divide the interval $(0,1)$ into sub-intervals with length $h:=1 / N$, so we have $N+1$ mesh points spaced by $h$ and numbered from 0 to $N$. The concentrations of $A$ and $B$ are viewed as homogeneous within each sub-interval, and diffusion through sub-interval boundaries is made as inflow/outflow reactions.

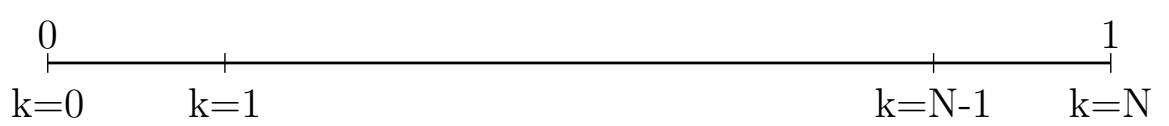

The boundary conditions in chemical systems usually represent the wall of a container; we need a condition that says that the chemicals can not leak through the walls of the container and there is no external input of chemical reactants to the system. Such boundary condition is called "zero-flux" boundary condition.

For the left end point $x=0$ at $k=0$ we use the forward difference approximation:

$$
\left.\partial_{x} a(t)\right|_{x=0} \approx \frac{a_{1}^{N}(t)-a_{0}^{N}(t)}{h}=0 .
$$

At $k=0$ we have $a_{0}^{N}(t)=a_{1}^{N}(t)$. Similarly, we have $b_{0}^{N}(t)=b_{1}^{N}(t)$.

For the right end point $x=1$ at $k=N$ we use the backward difference approximation:

$$
\left.\partial_{x} a(t)\right|_{x=1} \approx \frac{a_{N}^{N}(t)-a_{N+1}^{N}(t)}{h}=0 .
$$




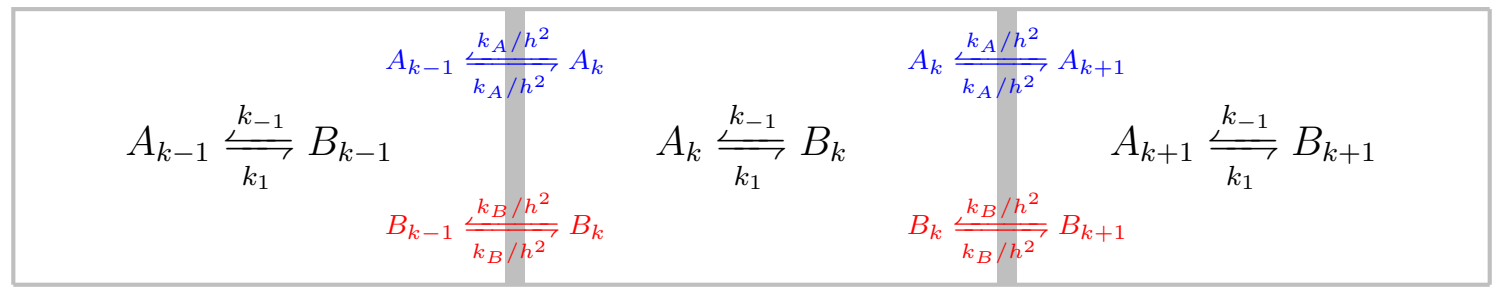

Figure 4.1: Boxes $k-1, k$ and $k+1$; linear case

At $k=N$ we have $a_{N+1}^{N}(t)=a_{N}^{N}(t)$. Similarly, we have $b_{N+1}^{N}(t)=b_{N}^{N}(t)$.

$$
\left\{\begin{array}{l}
\dot{a}_{k}^{N}(t)=-k_{1} a_{k}^{N}(t)+k_{-1} b_{k}^{N}(t)+\frac{k_{A}}{h^{2}}\left(a_{k-1}^{N}(t)-2 a_{k}^{N}(t)+a_{k+1}^{N}(t)\right), \\
\left.\dot{b}_{k}^{N}(t)=k_{1} a_{k}^{N}(t)-k_{-1} b_{k}^{N} t\right)+\frac{k_{B}}{h^{2}}\left(b_{k-1}^{N}(t)-2 b_{k}^{N}(t)+b_{k+1}^{N}(t)\right), \\
a_{k}^{N}(0)=\frac{1}{h} \int_{(k-1) h}^{k h} a_{0}(x) d x \\
b_{k}^{N}(0)=\frac{1}{h} \int_{(k-1) h}^{k h} b_{0}(x) d x \\
a_{0}^{N}(t)=a_{1}^{N}(t), \quad a_{N+1}^{N}(t)=a_{N}^{N}(t) \\
b_{0}^{N}(t)=b_{1}^{N}(t), \quad b_{N+1}^{N}(t)=b_{N}^{N}(t) .
\end{array}\right.
$$

To see what happens inside each sub-interval (box), we zoom into a box, say, with index $k$, and look what kind of reactions take places inside the box itself and with its neighbors. Inside the box labeled $k^{t h}$, there is a reaction $A_{k} \underset{k_{1}}{\stackrel{k_{-1}}{\leftrightarrows}} B_{k}$; in addition, there are inflow and outflow reactions between adjacent boxes, as shown in the Figure (4.1). If we combine all these reactions to evaluate the rate of change of the concentrations $a_{k}, b_{k}$ inside the $k^{t h}$ box, we have the following

$$
\begin{aligned}
& \dot{a}_{k}(t)=-k_{1} a_{k}(t)+k_{-1} b_{k}(t)+\frac{k_{A}}{h^{2}} a_{k-1}(t)-\frac{k_{A}}{h^{2}} a_{k}(t)+\frac{k_{A}}{h^{2}} a_{k+1}(t)-\frac{k_{A}}{h^{2}} a_{k}(t), \\
& \dot{b}_{k}(t)=-k_{1} a_{k}(t)+k_{-1} b_{k}(t)+\frac{k_{B}}{h^{2}} b_{k-1}(t)-\frac{k_{B}}{h^{2}} b_{k}(t)+\frac{k_{B}}{h^{2}} b_{k+1}(t)-\frac{k_{B}}{h^{2}} b_{k}(t) .
\end{aligned}
$$

We can rewrite them as following

$$
\dot{a}_{k}(t)=-k_{1} a_{k}(t)+k_{-1} b_{k}(t)+\frac{k_{A}}{h^{2}}\left(a_{k-1}(t)-2 a_{k}(t)+a_{k+1}(t)\right),
$$




$$
\dot{b}_{k}(t)=-k_{1} a_{k}(t)+k_{-1} b_{k}(t)+\frac{k_{B}}{h^{2}}\left(b_{k-1}(t)-2 b_{k}(t)+b_{k+1}(t)\right) .
$$

Thus we end up with the same equations (5.2) (note that $N$ is an index indicates to number of boxes), which give us a convincing reason to use the method of lines to approximate (5.1) and explain the connection between method of lines and chemical reaction-diffusion systems.

Now we present the general framework for the convergence analysis for the method of lines as in [44].

Consider a general equation of the type

$$
\partial_{t} v=\mathcal{F}(t, v), \quad v(0, x)=v_{0}(x), \quad x \in \mathbb{R}
$$

where $\mathcal{F}$ represents a partial differential operator which differentiates the unknown function (or a vector function) $v(t, x)$ with respect to its space variable $x \in \mathbb{R}, \mathcal{F}$ should satisfy some boundary conditions and $\mathcal{F}$ should not differentiate with respect to the time variable $t$. Now we define by a discretization of the space variable in (4.8) (e.g., by using the method of lines) an ODE system,

$$
\dot{u}(t)=F(t, u), 0<t \leq T, u(0)=u_{0}, x \in \mathbb{R}, \quad F(t, \cdot): \mathbb{R}^{N} \rightarrow \mathbb{R}^{N},
$$

where $u_{k}$ is the continuous time approximation to $u\left(t, x_{k}\right)$. We assume that $u$ and $F$ represent the values of grid functions on a space grid covering the space domain of (4.8). Furthermore, we let $\rho(h)$ refer to the grid spacing, i.e., to the grid distance which may vary over the grid (in our case, we have $\rho(h)=h=\frac{1}{N}$ ). We suppose that the two Cauchy problems (4.8) and (4.9) possess unique solutions $v(t, x)$ and $u(t, x)$, respectively. Also, it is supposed that the $v(t, x)$ is as smooth as the numerical analysis requires.

Let $r^{N}$ be the natural restriction operator to the space grid. We write $v^{N}(t)=r^{N} v(t, x)$. If the discretization in space is convergent, the space discretization error

$$
e^{N}(t)=u(t)-v^{N}(t)
$$

can be made arbitrarily small upon grid refinement. 


\section{Convergence of the Discretization in Space}

Consider the two Cauchy problems (4.8), (4.9). Introduce the space truncation error

$$
\varepsilon^{N}(t)=F\left(t, v^{N}(t)\right)-\dot{v}^{N}(t)
$$

where $\dot{v}^{N}(t)=\frac{d}{d t} v^{N}(t)=r^{N} \partial_{t} v$. The defect $\varepsilon^{N}$ is obtained by substituting the true solution $v^{N}$ into the semi-discrete approximation. Loosely speaking, it measures how the partial differential operator $\mathcal{F}$ is approximated by the vector function $F$. The consistency of the method for a given norm means that $\left\|\varepsilon^{N}(t)\right\| \rightarrow 0$ as $\rho(h) \rightarrow 0$ uniformly in $t$.

It now trivially follows that $e^{N}$, the discretization error in space, is a solution of the ODE system

$$
\dot{e}^{N}(t)=F\left(t, v^{N}+e^{N}\right)-F\left(t, v^{N}(t)\right)+\varepsilon^{N}(t), \quad 0<t \leq T .
$$

Using the mean value theorem for vector functions, we can write

$$
\begin{gathered}
\dot{e}^{N}(t)=M(t) e^{N}(t)+\varepsilon^{N}(t), 0<t \leq T, \\
M(t)=\int_{0}^{1} F^{\prime}\left(t, v^{N}+\theta e^{N}\right) d \theta
\end{gathered}
$$

where $F^{\prime}(t, \cdot)$ is the Jacobian matrix of $F(t, \cdot)$.

This result shows that $e^{N}(t)$ depends essentially on $\varepsilon^{N}(t)$, which is determined completely by the smoothness of $v$ and the quality of the approximation in space, and on the stability of the ODE system (4.9). We shall give a bound for $e^{N}$ from which convergence of $u$ to $v^{N}$ can be derived, provided that the discretization in space is consistent. This bound relies completely upon the fundamental concept of logarithmic matrix norm.

Introduce a norm $\|\cdot\|$ on $\mathbb{R}^{N}$. Let $\mu[\cdot]$ be its corresponding logarithmic matrix norm, defined by

$$
\mu[A]=\max _{\zeta \neq 0}<A \zeta, \zeta>/\|\zeta\|^{2},
$$

where $A=\left(a_{i j}\right)$ is a real $N \times N$ matrix. 
Let $T^{N}(t)=\left\{\zeta: \zeta=v^{N}(t)+\theta e^{N}(t), 0 \leq \theta \leq 1\right\}$ and let $\mu_{\max }$ be a constant such that

$$
\mu_{\max } \geq \max _{\zeta}\left\{\mu\left[F^{\prime}(t, \zeta)\right]: \zeta \in T^{N}(t)\right\} \text { for all } t \in[0, T]
$$

Hence for each $t$ we compute the maximal logarithmic matrix norm of $F^{\prime}$ on the line segment $T^{N}(t)$ and majorize these by $\mu_{\max }$. Then

$$
\left\|e^{N}(t)\right\| \leq e^{\mu_{\max } t}\left\|e^{N}(0)\right\|+\int_{0}^{t} e^{\mu_{\max }(t-\tau)}\left\|\varepsilon^{N}(\tau)\right\| d \tau
$$

If $\left\|e^{N}(0)\right\|=0$, we can write

$$
\left\|e^{N}(t)\right\| \leq C\left(t, \mu_{\max }\right) \max _{0 \leq \tau \leq t}\left\|\varepsilon^{N}(\tau)\right\|, 0 \leq t \leq T
$$

where $C\left(t, \mu_{\max }\right)=\left(e^{\mu_{\max } t}-1\right) / \mu_{\max }$ depends only on $t$ and $\mu_{\max }$. Consequently, if a constant $\mu_{\max }$ exists independent of the grid spacing, $\left\|e^{N}(t)\right\| \rightarrow 0$, for $\rho(h) \rightarrow 0$. Thus we can state

Theorem 4.2. [44] Suppose that the discretization in space is consistent and that $\mu_{\max }$ exists independent of the grid spacing. Then the discretization in space is convergent. 


\section{Chapter 5}

\section{Convergence of the Method of Lines:}

\section{linear case}

To prove convergence of the method of lines, we will start with the simple model

$$
A \underset{k_{1}}{\stackrel{k_{-1}}{\leftrightarrows}} B
$$

Recall: the one-dimensional chemical reaction-diffusion system of two species with Neumann boundary condition reads

$$
\left\{\begin{array}{l}
\partial_{t} a(t, x)=-k_{1} a(t, x)+k_{-1} b(t, x)+k_{A} \partial_{x}^{2} a(t, x) . \\
\partial_{t} b(t, x)=k_{1} a(t, x)-k_{-1} b(t, x)+k_{B} \partial_{x}^{2} b(t, x) . \\
a(0, x)=a_{0}(x), \\
b(0, x)=b_{0}(x), \\
\partial_{x} a(t, 0)=0 \text { and } \partial_{x} a(t, 1)=0, \\
\partial_{x} b(t, 0)=0 \text { and } \partial_{x} b(t, 1)=0,
\end{array}\right.
$$


and its semi-discrete scheme

$$
\left\{\begin{array}{l}
\dot{a}_{k}^{N}(t)=-k_{1} a_{k}^{N}(t)+k_{-1} b_{k}^{N}(t)+\frac{k_{A}}{h^{2}}\left(a_{k-1}^{N}(t)-2 a_{k}^{N}(t)+a_{k+1}^{N}(t)\right) . \\
\left.\dot{b}_{k}^{N}(t)=k_{1} a_{k}^{N}(t)-k_{-1} b_{k}^{N} t\right)+\frac{k_{B}}{h^{2}}\left(b_{k-1}^{N}(t)-2 b_{k}^{N}(t)+b_{k+1}^{N}(t)\right) . \\
a_{k}^{N}(0)=\frac{1}{h} \int_{(k-1) h}^{k h} a_{0}(x) d x \\
b_{k}^{N}(0)=\frac{1}{h} \int_{(k-1) h}^{k h} b_{0}(x) d x \\
a_{0}^{N}(t)=a_{1}^{N}(t), \quad a_{N+1}^{N}(t)=a_{N}^{N}(t) . \\
b_{0}^{N}(t)=b_{1}^{N}(t), \quad b_{N+1}^{N}(t)=b_{N}^{N}(t) .
\end{array}\right.
$$

Traditionally, the numerical PDE literature talks about the three facets: stability, consistency, and convergence. It is then customary to prove that any two of the three imply the third one, i.e., it is sufficient to look at any selection of two of the three. However, here we do not need to prove the convergence in that sense. Since (5.2) are linear, it is enough to prove weak convergence in $L^{2}(\Omega)$. Now we are ready to present the main result of this chapter.

Theorem 5.1. Let $a_{0}$ and $b_{0} \in L^{2}(0,1)$. Then the solution of (5.2) converges weakly in $L^{2}(\Omega)$ to a weak solution of (5.1).

Proof. Let $\Omega:=(0, T) \times(0,1)$ for fixed time $T$, and take the test function $\phi \in C_{c}^{\infty}(\Omega)$. $\mathrm{t}$

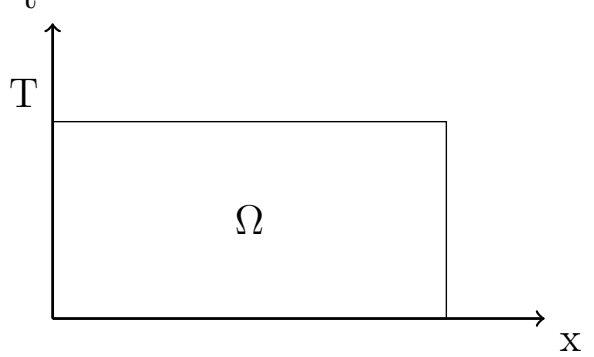

Denote

$$
\begin{cases}a^{N}(t, x):=a_{k}^{N}(t) & \text { for }(k-1) h \leq x<k h . \\ b^{N}(t, x):=b_{k}^{N}(t) & \text { for }(k-1) h \leq x<k h .\end{cases}
$$

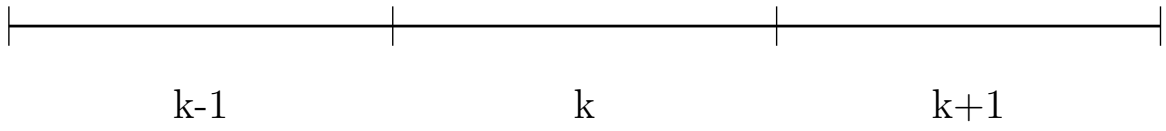


We can rewrite the system as following:

$$
\begin{aligned}
& \dot{a}^{N}(t, x)=-k_{1} a^{N}(t, x)+k_{-1} b^{N}(t, x)+\frac{k_{A}}{h^{2}}\left[a^{N}(t, x-h)-2 a^{N}(t, x)+a^{N}(t, x+h)\right], \\
& \dot{b}^{N}(t, x)=-k_{-1} b^{N}(t, x)+k_{1} a^{N}(t, x)+\frac{k_{B}}{h^{2}}\left[b^{N}(t, x-h)-2 b^{N}(t, x)+b^{N}(t, x+h)\right] .
\end{aligned}
$$

Multiply (5.3), (5.4) by $\phi(t, x)$ to get

$$
\begin{aligned}
\dot{a}^{N}(t, x) \phi(t, x)= & -k_{1} a^{N}(t, x) \phi(t, x)+k_{-1} b^{N}(t, x) \phi(t, x) \\
& +\frac{k_{A}}{h^{2}}\left[a^{N}(t, x-h)-2 a^{N}(t, x)+a^{N}(t, x+h)\right] \phi(t, x)
\end{aligned}
$$

and

$$
\begin{aligned}
\dot{b}^{N}(t, x) \phi(t, x)= & -k_{-1} b^{N}(t, x) \phi(t, x)+k_{1} a^{N}(t, x) \phi(t, x) \\
& +\frac{k_{B}}{h^{2}}\left[b^{N}(t, x-h)-2 b^{N}(t, x)+b^{N}(t, x+h)\right] \phi(t, x)
\end{aligned}
$$

Since $\phi$ has compact support, then $(\exists) \varepsilon>0$, such that $\phi(t, x)=0$ for all $x \in[0, \varepsilon]$ or

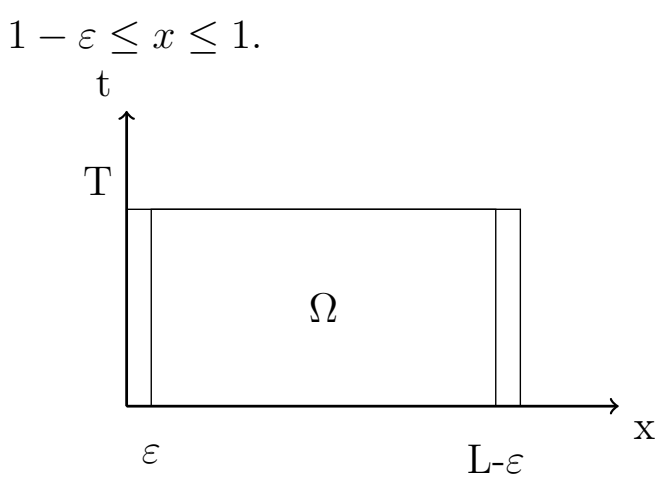

Now integrate both sides with respect to $x$ from 0 to 1 to obtain

$$
\begin{aligned}
\int_{0}^{1} \dot{a}^{N}(t, x) \phi(t, x) d x= & -k_{1} \int_{0}^{1} a^{N}(t, x) \phi(t, x) d x,+k_{-1} \int_{0}^{1} b^{N}(t, x) \phi(t, x) d x \\
& +\frac{k_{A}}{h^{2}} \int_{0}^{1}\left[a^{N}(t, x-h)-2 a^{N}(t, x)+a^{N}(t, x+h)\right] \phi(t, x) d x . \\
\int_{0}^{1} \dot{b}^{N}(t, x) \phi(t, x) d x= & -k_{-1} \int_{0}^{1} b^{N}(t, x) \phi(t, x) d x+k_{1} \int_{0}^{1} a^{N}(t, x) \phi(t, x) d x \\
& +\frac{k_{B}}{h^{2}} \int_{0}^{1}\left[b^{N}(t, x-h)-2 b^{N}(t, x)+b^{N}(t, x+h)\right] \phi(t, x) d x,
\end{aligned}
$$


We first compute the two integrals in the right hand side of (5.7) by the change of variables from $x$ coordinate to $y$ coordinate as following:

$$
\begin{aligned}
\int_{0}^{1} \phi(t, x) a^{N}(t, x-h) d x & =\int_{\varepsilon-h}^{1-\varepsilon-h} \phi(t, y+h) a^{N}(t, y) d y=\int_{0}^{1} \phi(t, y+h) a^{N}(t, y) d y \\
\int_{0}^{1} \phi(t, x) b^{N}(t, x-h) d x & =\int_{\varepsilon-h}^{1-\varepsilon-h} \phi(t, y+h) b^{N}(t, y) d y=\int_{0}^{1} \phi(t, y+h) b^{N}(t, y) d y .
\end{aligned}
$$

Similarly, we compute the two integrals on the right hand side of (5.8) to obtain

$$
\begin{aligned}
\int_{0}^{1} \phi(t, x) a^{N}(t, x+h) d x & =\int_{\varepsilon+h}^{1-\varepsilon+h} \phi(t, y-h) a^{N}(t, y) d y=\int_{0}^{1} \phi(t, y-h) a^{N}(t, y) d y . \\
\int_{0}^{1} \phi(t, x) b^{N}(t, x+h) d x & =\int_{\varepsilon+h}^{1-\varepsilon+h} \phi(t, y-h) b^{N}(t, y) d y=\int_{0}^{1} \phi(t, y-h) b^{N}(t, y) d y .
\end{aligned}
$$

Now we integrate both (5.7), (5.8) with respect to $t$ from 0 to $T$ to get

$$
\begin{gathered}
\int_{0}^{T} \int_{0}^{1} \dot{a}^{N}(t, x) \phi(t, x) d x d t \\
=-k_{1} \int_{0}^{T} \int_{0}^{1} a^{N}(t, x) \phi(t, x) d x d t+k_{-1} \int_{0}^{T} \int_{0}^{1} b^{N}(t, x) \phi(t, x) d x d t \\
+\left(k_{A}\right) \int_{0}^{T} \int_{0}^{1} a^{N}(t, x)\left[\frac{\phi(t, x+h)-2 \phi(t, x)+\phi(t, x-h)}{h^{2}}\right] d x d t \\
\int_{0}^{T} \int_{0}^{1} \dot{b}^{N}(t, x) \phi(t, x) d x d t=-k_{-1} \int_{0}^{T} \int_{0}^{1} b^{N}(t, x) \phi(t, x) d x d t \\
\quad+k_{1} \int_{0}^{T} \int_{0}^{1} a^{N}(t, x) \phi(t, x) d x d t \\
\quad+\left(k_{B}\right) \int_{0}^{1} b^{N}(t, x)\left[\frac{\phi(t, x+h)-2 \phi(t, x)+\phi(t, x-h)}{h^{2}}\right] d x d t .
\end{gathered}
$$

Next we need to pass to the limit as $N \rightarrow \infty$. So we need to prove $a^{N} \rightarrow a$ weakly in $L^{2}(\Omega)($ at least, up to a subsequence). Thus, it is enough to show $(\exists) C>0$ such that

$$
\int_{0}^{T} \int_{0}^{1}\left|a^{N}(t, x)\right|^{2} d x d t \leq C<\infty \quad(\forall) N .
$$


which means that

$$
\begin{aligned}
\int_{0}^{T} \int_{0}^{1}\left|a^{N}(t, x)\right|^{2} d x d t & =\int_{0}^{T} \sum_{k=1}^{N} \int_{(k-1) h}^{k h}\left|a_{k}^{N}(t)\right|^{2} d x d t \\
& =\int_{0}^{T} \sum_{k=1}^{N}\left|a_{k}^{N}(t)\right|^{2} \int_{(k-1) h}^{k h} d x d t \\
& =\int_{0}^{T} \sum_{k=1}^{N}\left|a_{k}^{N}(t)\right|^{2}[k h-(k-1) h] d t \\
& =\int_{0}^{T} \sum_{k=1}^{N}\left|a_{k}^{N}(t)\right|^{2} h d t \\
& =h \sum_{k=1}^{N} \int_{0}^{T}\left|a_{k}^{N}(t)\right|^{2} d t .
\end{aligned}
$$

Thus, we just need to show that the last quantity is bounded from above, i.e.,

$$
h \sum_{k=1}^{N} \int_{0}^{T}\left|a_{k}^{N}(t)\right|^{2} d t<\infty .
$$

Similarly we need to show $b^{N} \rightarrow b$ weakly in $L^{2}(\Omega)$ as $N \rightarrow \infty$ (possibly, up to subsequence). So, it is enough to show

$$
\int_{0}^{T} \int_{0}^{1}\left(\left|a^{N}(t, x)\right|^{2}+\left|b^{N}(t, x)\right|^{2}\right) d x d t=h \sum_{k=1}^{N} \int_{0}^{T}\left(\left|a_{k}^{N}(t)\right|^{2}+\left|b_{k}^{N}(t)\right|^{2}\right) d t
$$

is bounded from above.

Back to the equations (5.3) and (5.4), we rewrite them as functions in $t$ in this way:

$$
\begin{aligned}
& \dot{a}_{k}^{N}(t)=-k_{1} a_{k}^{N}(t)+k_{-1} b_{k}^{N}(t)+\frac{k_{A}}{h^{2}}\left[a_{k-1}^{N}(t)-2 a_{k}^{N}(t)+a_{k+1}^{N}(t)\right], \\
& \dot{b}_{k}^{N}(t)=-k_{-1} b_{k}^{N}(t)+k_{1} a_{k}^{N}(t)+\frac{k_{B}}{h^{2}}\left[b_{k-1}^{N}(t)-2 b_{k}^{N}(t)+b_{k+1}^{N}(t)\right] .
\end{aligned}
$$

Multiply (5.12) by $a_{k}^{N}(t)$ and (5.13) by $b_{k}^{N}(t)$, getting

$$
\begin{aligned}
& a_{k}^{N}(t) \dot{a}_{k}^{N}(t)=-k_{1}\left(a_{k}^{N}(t)\right)^{2}+k_{-1} a_{k}^{N}(t) b_{k}^{N}(t)+\frac{k_{A}}{h^{2}}\left[a_{k}^{N}(t) a_{n-1}^{N}(t)-2\left(a_{k}^{N}(t)\right)^{2}+a_{k}^{N}(t) a_{k+1}^{N}(t)\right], \\
& b_{k}^{N}(t) \dot{b}_{k}^{N}(t)=-k_{-1}\left(b_{k}^{N}(t)\right)^{2}+k_{1} a_{k}^{N}(t) b_{k}^{N}(t)+\frac{k_{B}}{h^{2}}\left[b_{k}^{N}(t) b_{k-1}^{N}(t)-2\left(b_{k}^{N}(t)\right)^{2}+b_{k}^{N}(t) b_{k+1}^{N}(t)\right] .
\end{aligned}
$$


Note that the left hand sides of the above equations equal $\frac{1}{2} \frac{d}{d t}\left(a_{k}^{N}(t)\right)^{2}$ and $\frac{1}{2} \frac{d}{d t}\left(b_{k}^{N}(t)\right)^{2}$, respectively.

Before going any further let us do some algebra computations to simplify the equations. For the time being, we will drop the $t$ and $N$ notations for simplicity.

We know

$$
\begin{gathered}
a_{k} a_{k-1} \leq \frac{a_{k}^{2}+a_{k-1}^{2}}{2} \\
\Longrightarrow a_{k} a_{k-1}-2 a_{k}^{2}+a_{k} a_{k+1} \leq \frac{a_{k-1}^{2}+a_{k+1}^{2}-2 a_{k}^{2}}{2} .
\end{gathered}
$$

Similarly,

$$
\Longrightarrow b_{k} b_{k-1}-2 b_{k}^{2}+b_{k} b_{k+1} \leq \frac{b_{k-1}^{2}+b_{k+1}^{2}-2 b_{k}^{2}}{2} .
$$

Take the sum from $k=1$ to $N$, and divide by $h^{2}$, getting

$$
\begin{gathered}
\frac{1}{h^{2}} \sum_{k=1}^{N}\left(a_{k} a_{k-1}-2 a_{k}^{2}+a_{k} a_{k+1}\right) \leq \frac{1}{2 h^{2}} \sum_{k=1}^{N}\left(a_{k-1}^{2}+a_{k+1}^{2}-2 a_{k}^{2}\right), \leq 0 . \\
\frac{1}{h^{2}} \sum_{k=1}^{N}\left(b_{k} b_{k-1}-2 b_{k}^{2}+b_{k} b_{k+1}\right) \leq \frac{1}{2 h^{2}} \sum_{k=1}^{N}\left(b_{k-1}^{2}+b_{k+1}^{2}-2 b_{k}^{2}\right) . \leq 0 .
\end{gathered}
$$

By using the boundary conditions, we end up with the following inequalities:

$$
\begin{aligned}
& \frac{1}{2} \frac{d}{d t}\left(\sum_{k=1}^{N}\left(a_{k}^{N}(t)\right)^{2}\right) \leq-k_{1} \sum_{k=1}^{N}\left(a_{k}^{N}(t)\right)^{2}+k_{-1} \sum_{k=1}^{N}\left(a_{k}^{N}(t) b_{k}^{N}(t)\right), \\
& \frac{1}{2} \frac{d}{d t}\left(\sum_{k=1}^{N}\left(b_{k}^{N}(t)\right)^{2}\right) \leq-k_{-1} \sum_{k=1}^{N}\left(b_{k}^{N}(t)\right)^{2}+k_{1} \sum_{k=1}^{N}\left(a_{k}^{N}(t) b_{k}^{N}(t)\right) .
\end{aligned}
$$


After adding (5.14) and (5.15), we get

$$
\begin{aligned}
\frac{1}{2} \frac{d}{d t} \sum_{k=1}^{N}\left(\left(a_{k}^{N}(t)\right)^{2}+\left(b_{k}^{N}(t)\right)^{2}\right) & \leq-k_{1} \sum_{k=1}^{N}\left(a_{k}^{N}(t)\right)^{2}+\left(k_{1}+k_{-1}\right) \sum_{k=1}^{N}\left(a_{k}^{N}(t) b_{k}^{N}(t)\right)-k_{-1} \sum_{k=1}^{N}\left(b_{k}^{N}(t)\right)^{2} \\
& \leq-k_{1} \sum_{k=1}^{N}\left(a_{k}^{N}(t)\right)^{2}+\frac{\left(k_{1}+k_{-1}\right)}{2} \sum_{k=1}^{N}\left(\left(a_{k}^{N}(t)\right)^{2}+\left(b_{k}^{N}(t)\right)^{2}\right) \\
& -k_{-1} \sum_{k=1}^{N-1}\left(b_{k}^{N}(t)\right)^{2} \\
& \leq \frac{-\left(k_{1}-k_{-1}\right)}{2} \sum_{k=1}^{N}\left(a_{k}^{N}(t)\right)^{2}+\frac{\left(k_{1}-k_{-1}\right)}{2} \sum_{k=1}^{N}\left(b_{k}^{N}(t)\right)^{2} \\
& \leq \frac{\left|k_{1}-k_{-1}\right|}{2} \sum_{k=1}^{N}\left(\left(a_{k}^{N}(t)\right)^{2}+\left(b_{k}^{N}(t)\right)^{2}\right) .
\end{aligned}
$$

Denote

$$
\Psi(t):=\sum_{k=1}^{N}\left(\left(a_{k}^{N}(t)\right)^{2}+\left(b_{k}^{N}(t)\right)^{2}\right),
$$

so we have

$$
\frac{1}{2} \frac{d}{d t} \Psi(t) \leq \frac{\left|k_{1}-k_{-1}\right|}{2} \Psi(t) .
$$

By Gronwall's inequality we obtain

$$
\begin{gathered}
\Psi(t) \leq \Psi(0) \exp \left(\left|k_{1}-k_{-1}\right| T\right) \\
\Longleftrightarrow \sum_{k=1}^{N}\left(\left(a_{k}^{N}(t)\right)^{2}+\left(b_{k}^{N}(t)\right)^{2}\right) \leq \sum_{k=1}^{N}\left(\left(a_{k}^{N}(0)\right)^{2}+\left(b_{k}^{N}(0)\right)^{2}\right) \exp \left(\left|k_{1}-k_{-1}\right| T\right) .
\end{gathered}
$$

Thus,

$$
\begin{aligned}
\int_{0}^{T} \int_{0}^{1}\left(\left|a^{N}(t, x)\right|^{2}+\left|b^{N}(t, x)\right|^{2}\right) d x d t & =h \sum_{k=1}^{N} \int_{0}^{T}\left(\left|a_{k}^{N}(t)\right|^{2}+\left|b_{k}^{N}(t)\right|^{2}\right) d t \\
& \leq h \sum_{k=1}^{N} \int_{0}^{T}\left(\left(a_{k}^{N}(0)\right)^{2}+\left(b_{k}^{N}(0)\right)^{2}\right) \exp \left(\left|k_{1}-k_{-1}\right| T\right) d t .
\end{aligned}
$$

Since

$$
\left|a_{k}^{N}(0)\right|^{2}=\left(\frac{1}{h} \int_{(k-1) h}^{k h} a_{0}(x) d x\right)^{2}
$$


then by Hölder inequality

$$
\left|a_{k}^{N}(0)\right|^{2} \leq \frac{1}{h} \int_{(k-1) h}^{k h}\left(a_{0}(x)\right)^{2} d x .
$$

which implies

$$
h \sum_{k=1}^{N}\left|a_{k}^{N}(0)\right|^{2} \leq\left\|a_{0}\right\|_{L^{2}(0,1)}^{2} .
$$

Therefore,

$$
\int_{0}^{T} \int_{0}^{1}\left(\left|a^{N}(t, x)\right|^{2}+\left|b^{N}(t, x)\right|^{2}\right) d x d t \leq C(T)\left(\left\|a_{0}\right\|_{L^{2}(0,1)}^{2}+\left\|b_{0}\right\|_{L^{2}(0,1)}^{2}\right),
$$

where $C(T):=T \exp \left(\left|k_{1}-k_{-1}\right| T\right)$. Therefore,

$$
\left\|a^{N}\right\|_{L^{2}(\Omega)}^{2}+\left\|b^{N}\right\|_{L^{2}(\Omega)}^{2} \leq C(T)\left(\left\|a_{0}\right\|_{L^{2}(0,1)}^{2}+\left\|b_{0}\right\|_{L^{2}(0,1)}^{2}\right)
$$

Therefore, we have two subsequences $a^{N}, b^{N}$ (not relabelled) such that $a^{N} \rightarrow a$ weakly in $L^{2}(\Omega)$ and $b^{N} \rightarrow b$ weakly in $L^{2}(\Omega)$ as $N \rightarrow \infty$.

After integrating by parts to(5.9) and (5.10) we get

$$
\begin{aligned}
-\int_{0}^{T} \int_{0}^{1} \partial_{t} \phi(t, x) a^{N}(t, x) d x d t & =-k_{1} \int_{0}^{T} \int_{0}^{1} a^{N}(t, x) \phi(t, x) d x d t \\
& +k_{-1} \int_{0}^{T} \int_{0}^{1} b^{N}(t, x) \phi(t, x) d x d t \\
& +k_{A} \int_{0}^{T} \int_{0}^{1} a^{N}(t, x)\left[\frac{\phi(t, x+h)-2 \phi(t, x)+\phi(t, x-h)}{h^{2}}\right] d x d t
\end{aligned}
$$

$$
\begin{aligned}
-\int_{0}^{T} \int_{0}^{1} \partial_{t} \phi(t, x) b^{N}(t, x) d x d t & =-k_{-1} \int_{0}^{T} \int_{0}^{1} b^{N}(t, x) \phi(t, x) d x d t \\
& +k_{1} \int_{0}^{T} \int_{0}^{1} a^{N}(t, x) \phi(t, x) d x d t \\
& +k_{B} \int_{0}^{1} b^{N}(t, x)\left[\frac{\phi(t, x+h)-2 \phi(t, x)+\phi(t, x-h)}{h^{2}}\right] d x d t .
\end{aligned}
$$


Now pass to the limit as $N \rightarrow \infty$, in order to get

$$
\begin{aligned}
-\int_{0}^{T} \int_{0}^{1} \partial_{t} \phi(t, x) a(t, x) d x & d t=-k_{1} \int_{0}^{T} \int_{0}^{1} a(t, x) \phi(t, x) d x d t \\
+ & k_{-1} \int_{0}^{T} \int_{0}^{1} b(t, x) \phi(t, x) d x d t \\
& +k_{A} \int_{0}^{T} \int_{0}^{1} a(t, x) \partial_{x}^{2} \phi(t, x) d x d t
\end{aligned}
$$

Also,

$$
\begin{gathered}
-\int_{0}^{T} \int_{0}^{1} \partial_{t} \phi(t, x) b(t, x) d x d t=-k_{-1} \int_{0}^{T} \int_{0}^{1} b(t, x) \phi(t, x) d x d t \\
+k_{1} \int_{0}^{T} \int_{0}^{1} a(t, x) \phi(t, x) d x d t \\
+k_{B} \int_{0}^{1} b(t, x) \partial_{x}^{2} \phi(t, x) d x d t .
\end{gathered}
$$

Therefore, the solution $\left(a^{N}, b^{N}\right)^{T}$ of $(5.2)$ converges weakly in $L^{2}(\Omega)$ to the solution $(a, b)^{T}$ of (5.1). 


\section{Chapter 6}

\section{Convergence of the Method of Lines:}

\section{nonlinear case}

In this chapter we concentrate on the proof-of-concept reaction

$$
A+B \underset{k_{1}}{\stackrel{k_{-1}}{\leftrightarrows}} C
$$

Let $I:=(0,1)$. The primary concern of this work is the system of semi-linear parabolic partial differential equations

$$
\begin{cases}\partial_{t} a(t, x)=-k_{1} a(t, x) b(t, x)+k_{-1} c(t, x)+k_{A} \partial_{x}^{2} a(t, x) & \text { in }[0, T) \times I, \\ \partial_{t} b(t, x)=-k_{1} a(t, x) b(t, x)+k_{-1} c(t, x)+k_{B} \partial_{x}^{2} b(t, x) & \text { in }[0, T) \times I, \\ \partial_{t} c(t, x)=k_{1} a(t, x) b(t, x)-k_{-1} c(t, x)+k_{C} \partial_{x}^{2} c(t, x) & \text { in }[0, T) \times I\end{cases}
$$

together with the homogeneous Neumann boundary conditions

$$
\left\{\begin{array}{l}
\partial_{x} a(t, 0)=\partial_{x} a(t, 1)=0 \\
\partial_{x} b(t, 0)=\partial_{x} b(t, 1)=0 \\
\partial_{x} c(t, 0)=\partial_{x} c(t, 1)=0
\end{array}\right.
$$

Here $k_{1}, k_{-1}$ and $T$ are positive constants and $k_{A}, k_{B}$ and $k_{C}$ are the constant positive diffusion coefficients.

The problem should be well-posed once appropriate initial conditions $a(0, x)=a_{0}(x)$, 
$b(0, x)=b_{0}(x)$ and $c(0, x)=c_{0}(x)$ are given. In the case of reaction-diffusion systems, there are two different aspects of existence to consider: local (in time) existence and global (in time) existence of solutions. The existence question is, in general, difficult to deal with. The well-posedness for a general form of nonlinear parabolic system was obtained in [32]. In addition, they established existence and uniqueness for specific, three species systems when the diffusion coefficients are the same for all three species. In [14] the authors established global existence and uniqueness of solutions to $(E 1)$ with constants $k_{1}=k_{-1}=1$ and distinct diffusion coefficients $k_{A}, k_{B}, k_{C}$.

For (6.1) we adopt the following paradigm: we envision splitting the spatial domain into $N$ equal subintervals in each of which we treat the concentrations of the three species as approximately constant. This, of course, is a fairly reasonable assumption for large $N$. We assume that a version of (6.1) takes place in each cell (or "box") $k$ (see Figure 6.1 below), and the diffusion of any of the three species can be thought of as a reaction between adjacent replicas of the same species. The coefficients of these same-species reactions must be proportional to $N^{2}$ in order to get diffusion in the $N \rightarrow \infty$ limit (see also [27] for an explanation of this scaling). The plan is to show that the standard reaction-diffusion system corresponding to (6.1) is obtained from these approximating reaction systems in the $N \rightarrow \infty$ limit.

\subsubsection{Discretization by the Method of Lines (MOL)}

We now discretize $(E 1)$ in space only: more precisely, we use the standard three-point stencil to approximate the second-order spatial derivatives. Let $N \geq 2$ and divide the interval $[0,1]$ into $N$ subintervals of equal length $h:=1 / N$, so that we have $N+1$ mesh points spaced by $h$ and numbered from 0 to $N$ (see figure (6.1)). The discretized problem is 


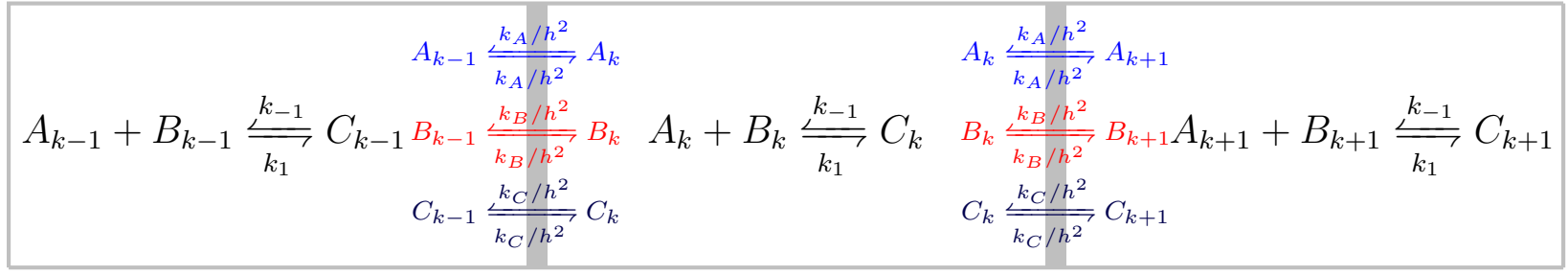

Figure 6.1: Boxes $k-1, k$ and $k+1$ : nonlinear case

$$
\left\{\begin{array}{l}
\dot{a}_{k}^{N}(t)=-k_{1} a_{k}^{N}(t) b_{k}^{N}(t)+k_{-1} c_{k}^{N}(t)+\frac{k_{A}}{h^{2}}\left[a_{k-1}^{N}(t)-2 a_{k}^{N}(t)+a_{k+1}^{N}(t)\right] \\
\dot{b}_{k}^{N}(t)=-k_{1} a_{k}^{N}(t) b_{k}^{N}(t)+k_{-1} c_{k}^{N}(t)+\frac{k_{B}}{h^{2}}\left[b_{k-1}^{N}(t)-2 b_{k}^{N}(t)+b_{k+1}^{N}(t)\right] \\
\dot{c}_{k}^{N}(t)=k_{1} a_{k}^{N}(t) b_{k}^{N}(t)-k_{-1} c_{k}^{N}(t)+\frac{k_{C}}{h^{2}}\left[c_{k-1}^{N}(t)-2 c_{k}^{N}(t)+c_{k+1}^{N}(t)\right] \\
a_{k}^{N}(0)=N \int_{(k-1) / N}^{k / N} a_{0}(x) d x \\
b_{k}^{N}(0)=N \int_{(k-1) / N}^{k / N} b_{0}(x) d x \\
c_{k}^{N}(0)=N \int_{(k-1) / N}^{k / N} c_{0}(x) d x \\
a_{0}^{N}(t):=a_{1}^{N}(t), b_{0}^{N}(t):=b_{1}^{N}(t) \text { and } c_{0}^{N}(t):=c_{1}^{N}(t), \\
a_{N+1}^{N}(t):=a_{N}^{N}(t), b_{N+1}^{N}(t):=b_{N}^{N}(t) \text { and } c_{N+1}^{N}(t):=c_{N}^{N}(t)
\end{array}\right.
$$

for $k=1, \ldots, N$, and $\left(\cdot=\frac{d}{d t}\right)$. For the left endpoint $x=0(k=0)$ we use the forward difference approximation

$$
\partial_{x} a(0, t) \approx \frac{a_{1}^{N}(t)-a_{0}^{N}(t)}{h}=0
$$

so we assume $a_{0}^{N}(t)=a_{1}^{N}(t)$. For the right endpoint $x=1(k=N)$ we use the backward difference approximation

$$
\partial_{x} a(1, t) \approx \frac{a_{N}^{N}(t)-a_{N+1}^{N}(t)}{h}=0
$$

which gives $a_{N+1}^{N}(t)=a_{N}^{N}(t)$. The same hold for b and c.

Define a time-dependent vector (a solution of $(E 2)$ )

$$
\vec{u}^{N}(t):=\left[\vec{a}^{N}(t), \vec{b}^{N}(t), \vec{c}^{N}(t)\right]^{T} \in \mathbb{R}^{3 N}
$$

where

$$
\vec{a}^{N}(t)=\left[a_{1}^{N}(t), \ldots, a_{N}^{N}(t)\right]^{T} \in \mathbb{R}^{N},
$$




$$
\begin{aligned}
& \vec{b}^{N}(t)=\left[b_{1}^{N}(t), \ldots, b_{N}^{N}(t)\right]^{T} \in \mathbb{R}^{N} \\
& \vec{c}^{N}(t)=\left[c_{1}^{N}(t), \ldots, c_{N}^{N}(t)\right]^{T} \in \mathbb{R}^{N} .
\end{aligned}
$$

What we presented above is known as Method of Lines (MOL) [39]; this nomenclature comes from the fact that we have reduced the original problem of finding a solution for $(E 1)$ at all points in the space-time rectangular domain $I \times[0, T]$ to the problem of finding a solution $\vec{u}^{N}$ on a finite number of lines in the space-time domain. By this method we store the concentrations at $N+1$ mesh points spaced by $h$ and numbered 0 to $N$, and estimate the second derivatives of these concentrations at every point by using these values. The result of carrying out this procedure is a discretization of the system. The discretization is a set of ODEs (E2) which formally reduce to the original PDE $(E 1)$ in the $N \rightarrow \infty$ limit. Note that this method is also called semi-discretization because $(E 1)$ is discretized in space only.

Define the following piecewise constant functions

$a^{N}(t, x):=a_{k}^{N}(t), \quad b^{N}(t, x):=b_{k}^{N}(t), \quad c^{N}(t, x):=c_{k}^{N}(t) \quad$ if $t>0$ and $(k-1) / N \leq x<k / N$.

Let $\|\cdot\|_{2}$ denote the $L^{2}(0,1)$-norm. We are now ready to present the main result of our paper.

Theorem 6.1. Assume that the initial data $a_{0}, b_{0}$ and $c_{0}$ in $L^{\infty}(0,1)$ such that $a_{0} \geq 0, b_{0} \geq 0$ and $c_{0} \geq 0$ a.e. in $(0,1)$. Then the solution $\vec{u}^{N}$ of $(E 2)$ converges in $L^{2}(0,1)$ to the solution $(\alpha, \beta, \gamma)$ of $(E 1)$ in the sense

$$
\lim _{N \rightarrow \infty}\left[\left\|a^{N}(t, \cdot)-\alpha(t, \cdot)\right\|_{2}+\left\|b^{N}(t, \cdot)-\beta(t, \cdot)\right\|_{2}+\left\|c^{N}(t, \cdot)-\gamma(t, \cdot)\right\|_{2}\right]=0
$$

for all $t \in[0, T)$.

We need to prove a comarison priciple and it is necessary to show that the scheme is consistent with the continuous problem, and that the discretized version of the spatial differential operator retains sufficient dissipative properties in order to allow an application of Gronwall's Lemma to the error term. As shown in [44], a uniform (in time) consistency estimate is sufficient to obtain convergence; however, the consistency estimate we proved is not uniform for small time, so we cannot directly employ the results in [44] to prove 
convergence in our case.

\subsection{The Comparison Principle (Continuous and semi- discrete Problems)}

Chen, Li and Wright [14] established a maximum principle for a version of $(E 1)$ on the whole real line and the constants $k_{-1}=k_{1}=1, k_{A}>0, k_{B}>0, k_{C}>0$. We adapt their proof to our case; the adaptation is quite straightforward but we show it here in full detail, mainly because the same proof will work for the discrete problem $(E 2)$ if one replaces the Heat Kernel by its discrete version.

We change the variables by setting

$$
u_{1}(t, x):=k_{1} a(t, x), \quad u_{2}(t, x):=k_{1} b(t, x), u_{3}(t, x):=k_{1} c(t, x)
$$

so that $(E 1)$ becomes

$$
\left\{\begin{array}{l}
\partial_{t} u_{1}(t, x)=-u_{1}(t, x) u_{2}(t, x)+k_{-1} u_{3}(t, x)+k_{A} \partial_{x}^{2} u_{1}(t, x), \\
\partial_{t} u_{2}(t, x)=-u_{1}(t, x) u_{2}(t, x)+k_{-1} u_{3}(t, x)+k_{B} \partial_{x}^{2} u_{2}(t, x), \\
\partial_{t} u_{3}(t, x)=u_{1}(t, x) u_{2}(t, x)-k_{-1} u_{3}(t, x)+k_{C} \partial_{x}^{2} u_{3}(t, x) .
\end{array}\right.
$$

and the boundary conditions become

$$
\left\{\begin{array}{l}
\partial_{x} u_{1}(t, 0)=\partial_{x} u_{1}(t, 1)=0 \\
\partial_{x} u_{2}(t, 0)=\partial_{x} u_{2}(t, 1)=0 \\
\partial_{x} u_{3}(t, 0)=\partial_{x} u_{3}(t, 1)=0
\end{array}\right.
$$

We also know that (see [14] or [37]) the solutions $u_{1}, u_{2}, u_{3}$ stay nonnegative if the initial data $u_{1,0}, u_{2,0}$ and $u_{3,0}$ are nonnegative.

The following lemma, adapted from [14], will be used in the proof of Theorem (6.3).

Lemma 6.2. Assume that $u_{1,0}, u_{2,0}$ and $u_{3,0}$ are nonnegative. Then there exists a constant 
C such that

$$
\int_{0}^{1} u_{i}(t, x) d x \leq C \quad \text { for all } t \text { and for } i=1,2,3 .
$$

Proof. First construct a linear combination $u_{1}+u_{2}+2 u_{3}$ from the three equations in (6.4), we obtain

$$
\partial_{t}\left(u_{1}(t, x)+u_{2}(t, x)+2 u_{3}(t, x)\right)=k_{A} \partial_{x}^{2} u_{1}(t, x)+k_{B} \partial_{x}^{2} u_{2}(t, x)+2 k_{C} \partial_{x}^{2} u_{3}(t, x)
$$

integrate both sides with respect to $x$ from 0 to 1 , we get

$$
\begin{aligned}
\partial_{t} \int_{0}^{1}\left(u_{1}(t, x)+u_{2}(t, x)+2 u_{3}(t, x)\right) d x= & k_{A} \int_{0}^{1} \partial_{x}^{2} u_{1}(t, x) d x+k_{B} \int_{0}^{1} \partial_{x}^{2} u_{2}(t, x) d x \\
& +2 k_{C} \int_{0}^{1} \partial_{x}^{2} u_{3}(t, x) d x
\end{aligned}
$$

using the boundary conditions (6.5), we have

$$
\partial_{t} \int_{0}^{1}\left(u_{1}(t, x)+u_{2}(t, x)+2 u_{3}(t, x)\right) d x=0
$$

It follows that

$$
\int_{0}^{1}\left(u_{1}(t, x)+u_{2}(t, x)+2 u_{3}(t, x)\right) d x=\int_{0}^{1}\left(u_{1,0}(x)+u_{2,0}(x)+2 u_{3,0}(x)\right) d x=C .
$$

Since all $u_{1}, u_{2}, u_{3}$ are nonnegative [14], we end up with

$$
\int_{0}^{1} u_{i}(t, x) d x \leq C
$$

for all $t$ and for all $i=1,2,3$.

Theorem 6.3. If the initial data $a_{0}, b_{0}, c_{0}$ of the system (6.4) belong to $L^{\infty}(0,1)$, then the solution of (6.4) is uniformly bounded for all time.

Proof. Let $H_{i}(t, x, y)$ be the Neumann Heat Kernel of the linear parabolic equation

$$
\partial_{t} v=\mathcal{L}_{i} v
$$

where $\mathcal{L}_{i}=k_{i} \partial_{x}^{2}$ for $i=1,2,3$ such that $k_{1}=k_{A}, k_{2}=k_{B}$, and $k_{3}=k_{C}$. Note that $H_{i}(t, x, y)=H\left(k_{i} t, x, y\right)$, where $H$ is the Neumann Heat Kernel defined in Appendix. 
First, from the nonnegativity of the solutions, we have

$$
\partial_{t} u_{1}-k_{A} \partial_{x}^{2} u_{1}=-u_{1} u_{2}+k_{-1} u_{3}
$$

which implies

$$
\partial_{t} u_{1}-k_{A} \partial_{x}^{2} u_{1} \leq k_{-1} u_{3}
$$

For each fixed $T>0$, we compare $u_{1}(T+\cdot, \cdot)$ with the solution of the linear equation

$$
\left\{\begin{array}{l}
\partial_{t} v-\mathcal{L}_{1} v=k_{-1} u_{3}(T+t, x) \\
v(0, x)=u_{1}(T, x)
\end{array}\right.
$$

We know that the solution of $(6.8)$ is

$$
v(T+t, x)=\int_{I} H\left(k_{A} t, x, y\right) u_{1}(y, T) d y+\int_{0}^{t} \int_{I} H\left(k_{A}(t-s), x, y\right) k_{-1} u_{3}(T+s, y) d y d s
$$

Thus,

$$
\begin{aligned}
u_{1}(T+t, x) & \leq \int_{I} H\left(k_{A} t, x, y\right) u_{1}(T, y) d y+\int_{0}^{t} \int_{I} H\left(k_{A}(t-s), x, y\right) k_{-1} u_{3}(T+s, y) d y d s \\
& =: I_{1}+I_{2} .
\end{aligned}
$$

When $t$ is bounded away from $0, H(t, x, y)$ is bounded in a pointwise sense. In the following, we assume that

$$
\frac{\delta}{2} \leq t \leq \delta \text { for some } \delta>0
$$

According to (7.18) (Appendix), the Neumann Heat Kernel satisfies the bounds

$$
0 \leq H(t, x, y) \leq 1+2 f(4 t) \leq 1+2 f(2 \delta) \text { for all } x, y \in I, t \geq \delta / 2
$$

where $f$ is defined in Appendix (7.1.4). 
Therefore, the integral $I_{1}$ can be easily bounded as

$$
\begin{aligned}
I_{1} & =\int_{I} H_{1}(t, x, y) u_{1}(T, y) d y \\
& \leq\left[1+2 f\left(2 k_{A} \delta\right)\right] \int_{I} u_{1}(T, y) d y \\
& \leq C\left[1+2 f\left(2 k_{A} \delta\right)\right],
\end{aligned}
$$

where, by Lemma 6.2, $C$ is a constant which is independent of $T$. As for the integral $I_{2}$, we can rewrite it as

$$
\begin{aligned}
I_{2} & =\int_{0}^{t} \int_{I} H_{1}(t-s, x, y) k_{-1} u_{3}(T+s, y) d y d s \\
& =\int_{0}^{t-\frac{\delta}{2}} \int_{I} H_{1}(t-s, x, y) k_{-1} u_{3}(T+s, y) d y d s+\int_{t-\frac{\delta}{2}}^{t} \int_{I} H_{1}(t-s, x, y) k_{-1} u_{3}(T+s, y) d y d s \\
& =: \hat{I}_{2}+\tilde{I}_{2} .
\end{aligned}
$$

Next we estimate

$$
\begin{aligned}
\hat{I}_{2} & =\int_{0}^{t-\frac{\delta}{2}} \int_{I} H_{1}(t-s, x, y) k_{-1} u_{3}(T+s, y) d y d s \\
& \leq \int_{0}^{t-\frac{\delta}{2}}\left(1+2 f\left(4 k_{A}(t-s)\right)\right) k_{-1} \int_{I} u_{3}(T+s, y) d y d s \\
& \leq k_{-1} C \int_{0}^{t-\frac{\delta}{2}}\left(1+2 f\left(4 k_{A}(t-s)\right)\right) d s \\
& \leq \frac{k_{-1} C \delta}{2}+\frac{k_{-1} C}{2 k_{A}} \int_{2 k_{A} \delta}^{4 k_{A} \delta} f(\tau) d \tau=O(\delta) .
\end{aligned}
$$

For the integral $\tilde{I}_{2}$, we use Hölder's inequality to get, for any $\infty>p, q>1$ such that 
$1 / p+1 / q=1$

$$
\begin{aligned}
\tilde{I}_{2} & =\int_{t-\frac{\delta}{2}}^{t} \int_{I} H_{1}(t-s, x, y) k_{-1} u_{3}(T+s, y) d y d s \\
& \leq \int_{t-\frac{\delta}{2}}^{t}\left|\int_{I} H_{1}(t-s, x, y) k_{-1} u_{3}(T+s, y) d y\right| d s \\
& \leq k_{-1} \int_{t-\frac{\delta}{2}}^{t}\left(\int_{I}\left|H_{1}(t-s, x, y)\right|^{q} d y\right)^{1 / q}\left(\int_{I}\left|u_{3}(T+s, y)\right|^{p} d y\right)^{1 / p} d s \\
& \leq k_{-1} \int_{t-\frac{\delta}{2}}^{t}\left(\int_{I}\left|1+2 f\left(4 k_{A}(t-s)\right)\right|^{q} d y\right)^{1 / q}\left(\int_{I}\left|u_{3}(T+s, y)\right|\left|u_{3}(T+s, y)\right|^{p-1} d y\right)^{1 / p} d s \\
& \leq k_{-1} \int_{t-\frac{\delta}{2}}^{t}\left|1+2 f\left(4 k_{A}(t-s)\right)\right|\left(\left\|u_{3}(T+s, \cdot)\right\|_{\infty}^{p-1} \int_{I}\left|u_{3}(T+s, y)\right| d y\right)^{1 / p} d s \\
& \leq C k_{-1} \max _{t-\frac{\delta}{2} \leq s \leq t}\left\|u_{3}(T+s, \cdot)\right\|_{\infty}^{\frac{p-1}{p}} \int_{t-\frac{\delta}{2}}^{t}\left(1+2 f\left(4 k_{A}(t-s)\right)\right) d s \\
& \leq C k_{-1}\left(\frac{\delta}{2}+\frac{1}{2 k_{A}} \int_{0}^{2 k_{A} \delta} f(\tau) d \tau\right)_{t-\frac{\delta}{2} \leq s \leq t} \max _{0}\left\|u_{3}(T+s, \cdot)\right\|_{\infty}^{\frac{p-1}{p}} .
\end{aligned}
$$

Therefore, we get

$$
u_{1}(T+t, x) \leq C+C \max _{t-\frac{\delta}{2} \leq s \leq t}\left\|u_{3}(T+s, \cdot)\right\|_{\infty}^{\frac{p-1}{p}},
$$

where the constant $C_{1}$ depends on $\delta$ (in fact, due to the integrability of $f, C_{1}$ tends to zero as $\delta$ tends to zero). Since $\frac{\delta}{2} \leq t \leq \delta$, we deduce

$$
\left.\max _{T+\frac{\delta}{2} \leq s \leq T+\delta} \| u_{1}(s, \cdot)\right)\left\|_{\infty} \leq C+C \max _{T \leq s \leq T+\delta}\right\| u_{3}(s, \cdot) \|_{\infty}^{\frac{p-1}{p}} .
$$

Applying the same argument to the equation for $u_{2}$ from the system (6.4), we also have

$$
\left.\max _{T+\frac{\delta}{2} \leq s \leq T+\delta} \| u_{2}(s, \cdot)\right)\left\|_{\infty} \leq C+C \max _{T \leq s \leq T+\delta}\right\| u_{3}(s, \cdot) \|_{\infty}^{\frac{p-1}{p}} .
$$

Finally, from the third equation of the system (6.4), we have

$$
\partial_{t} u_{3}-k_{C} \partial_{x}^{2} u_{3}=u_{1} u_{2}-k_{-1} u_{3}
$$

which implies

$$
\partial_{t} u_{3}-k_{C} \partial_{x}^{2} u_{3} \leq u_{1} u_{2}
$$


Just as before, one gets

$$
u_{3}(T+t, x) \leq C+C \max _{t-\frac{\delta}{2} \leq s \leq t}\left\|u_{1}(T+s, \cdot)\right\|_{\infty}\left\|u_{2}(T+s, \cdot)\right\|_{\infty}^{(p-1) / p},
$$

which yields

$$
\left.\max _{T+\frac{\delta}{2} \leq s \leq T+\delta} \| u_{3}(s, \cdot)\right)\left\|_{\infty} \leq C+C \max _{T \leq s \leq T+\delta}\right\| u_{1}(s, \cdot)\left\|_{\infty}\right\| u_{2}(s, \cdot) \|_{\infty}^{(p-1) / p} .
$$

As in [14], we can use (6.9)-(6.11) to get

$$
\left.\max _{T+\frac{\delta}{2} \leq s \leq T+\delta} \| u_{3}(s, \cdot)\right)\left\|_{\infty} \leq C+C \max _{T-\frac{\delta}{2} \leq s \leq T+\delta}\right\| u_{3}(s, \cdot) \|_{\infty}^{\omega}
$$

where

$$
\omega:=\frac{p-1}{p}+\left(\frac{p-1}{p}\right)^{2}
$$

We choose

$$
1<p<\frac{2}{3-\sqrt{5}}, \text { so that } 0<\omega<1
$$

Once more, as in [14], we infer

$$
\left.\max _{T+\frac{\delta}{2} \leq s \leq T+\delta} \| u_{3}(s, \cdot)\right) \|_{\infty} \leq C+C\left(\max _{T-\frac{\delta}{2} \leq s \leq T+\frac{\delta}{2}}\left\|u_{3}(s, \cdot)\right\|_{\infty}^{\omega}+\max _{T+\frac{\delta}{2} \leq s \leq T+\delta}\left\|u_{3}(s, \cdot)\right\|_{\infty}^{\omega}\right)
$$

which implies

$$
\left.\max _{T+\frac{\delta}{2} \leq s \leq T+\delta} \| u_{3}(s, \cdot)\right)\left\|_{\infty} \leq C+C_{2} \max _{T-\frac{\delta}{2} \leq s \leq T+\frac{\delta}{2}}\right\| u_{3}(s, \cdot) \|_{\infty}^{\omega}
$$

Denote

$$
M(t):=\max _{t-\frac{\delta}{2} \leq s \leq t+\frac{\delta}{2}}\left\|u_{3}(s, \cdot)\right\|_{\infty}^{\omega} .
$$

Then, one can derive (see [14]) from (6.14) that

$$
M(t) \leq C_{2}+C_{3}\left[M\left(t-\frac{\delta}{2}\right)\right]^{\omega} \text { for all } \frac{\delta}{2} \leq t \leq \delta
$$

Since $0<\omega<1$, we deduce $u_{3}$ is bounded for all time if $M\left(\frac{\delta}{2}\right)<\infty$, and thus, $u_{1}$ and $u_{2}$ are also bounded for all time. 
Note carefully that the proof of the Theorem 6.3 goes through if we replace $H$ by $H^{N}$ (i.e. the discrete Neumann Heat Kernel on I). Indeed, as shown in Appendix, Subsection 7.1.4, $H^{N}$ has all the desired properties.

Theorem 6.4. Assume that the initial data $a_{0}, b_{0}, c_{0}$ in the system (E2) are essentially bounded by a positive constant $M$. Then there exists a finite constant $\tilde{M}$ such that for all $N, k=1,2, \ldots, N$ and $t \in(0, \infty)$ we have

$$
\begin{aligned}
& 0 \leq a_{k}^{N}(t) \leq \tilde{M}, \\
& 0 \leq b_{k}^{N}(t) \leq \tilde{M}, \\
& 0 \leq c_{k}^{N}(t) \leq \tilde{M} .
\end{aligned}
$$

Proof. We can apply the same proof above, but replacing the Heat Kernel $H$ by $H^{N}$ and using (7.9), (7.10) and (7.11).

\subsection{Convergence}

In this section we will prove the main result. We first need to check the consistency of the MOL scheme when applied to our system.

We solve $(E 1)$ with initial data $a_{0}, b_{0}, c_{0}$, and denote by $(\alpha, \beta, \gamma)$ a solution. Let $N \geq 2$ be integer. For each $t \geq 0$, we define the vectors $\vec{\alpha}^{N}(t), \vec{\beta}^{N}(t), \vec{\gamma}^{N}(t)$ by

$$
\begin{aligned}
& \alpha_{k}^{N}(t):=\alpha(t,(k-1) / N) \text { for all } k=1, \ldots, N, \\
& \beta_{k}^{N}(t):=\beta(t,(k-1) / N) \text { for all } k=1, \ldots, N, \\
& \gamma_{k}^{N}(t):=\gamma(t,(k-1) / N) \text { for all } k=1, \ldots, N .
\end{aligned}
$$

Based on these vectors we now define the following piecewise constant functions

$$
\alpha^{N}(t, x):=\alpha_{k}^{N}(t), \beta^{N}(t, x):=\beta_{k}^{N}(t), \gamma^{N}(t, x):=\gamma_{k}^{N}(t) \text { if } t>0 \text { and }(k-1) / N \leq x<k / N
$$


and let

$$
\vec{v}^{N}(t)=\left[\vec{\alpha}^{N}(t), \vec{\beta}^{N}(t), \vec{\gamma}^{N}(t)\right]^{T} \in \mathbb{R}^{3 N}
$$

Also, let us denote the discrete Laplacian matrix with Neumann boundary condition on $I$ by

$$
\Delta^{N}:=N^{2}\left[\begin{array}{ccccccc}
-1 & 1 & 0 & 0 & 0 & \ldots & 0 \\
1 & -2 & 1 & 0 & 0 & \ldots & 0 \\
0 & 1 & -2 & 1 & 0 & \ldots & 0 \\
\vdots & \vdots & \vdots & \vdots & \vdots & \vdots & \vdots \\
0 & 0 & \ldots & 0 & 1 & -2 & 1 \\
0 & 0 & \ldots & 0 & 0 & 1 & -1
\end{array}\right]_{N \times N}
$$

Next, consider the vector field $\widetilde{F}^{N}: \mathbb{R}^{3 N} \rightarrow \mathbb{R}^{3 N}$ given by

$$
\widetilde{F}^{N}=\left[\begin{array}{c}
F^{N} \\
F^{N} \\
-F^{N}
\end{array}\right]
$$

where $F^{N}: \mathbb{R}^{N} \rightarrow \mathbb{R}^{N}$ is given by (in terms of a generic vector $\vec{\omega}^{N}=\left[\vec{x}^{N}, \vec{y}^{N}, \vec{z}^{N}\right]^{T} \in \mathbb{R}^{3 N}$ )

$$
F^{N}\left(\vec{\omega}^{N}\right)=\left[\begin{array}{c}
-k_{1} x_{1}^{N} y_{1}^{N}+k_{-1} z_{1}^{N} \\
\vdots \\
-k_{1} x_{N}^{N} y_{N}^{N}+k_{-1} z_{N}^{N}
\end{array}\right] .
$$

Let $\tilde{\Delta}^{N}$ be the $(3 N) \times(3 N)$ block-diagonal matrix whose diagonal blocks are the matrices $k_{A} \Delta^{N}, k_{B} \Delta^{N}$ and $k_{C} \Delta^{N}$ (where $\Delta^{N}$ is the matrix (6.17)). Note that the discrete system (E2) can now be written as

$$
\frac{d}{d t} \vec{u}^{N}(t)=\widetilde{F}^{N}\left(\vec{u}^{N}(t)\right)+\tilde{\Delta}^{N} \vec{u}^{N}(t)
$$

and it consists of three coupled systems

$$
\dot{\vec{a}}^{N}(t)=F^{N}\left(\vec{u}^{N}(t)\right)+k_{A} \Delta^{N} \vec{a}^{N}(t),
$$




$$
\begin{gathered}
\dot{\vec{b}}^{N}(t)=F^{N}\left(\vec{u}^{N}(t)\right)+k_{B} \Delta^{N} \vec{b}^{N}(t), \\
\dot{\vec{c}}^{N}(t)=-F^{N}\left(\vec{u}^{N}(t)\right)+k_{C} \Delta^{N} \vec{c}^{N}(t) .
\end{gathered}
$$

\subsubsection{A consistency estimate}

We begin by proving an estimate on the space truncation error; this is called a consistency estimate. This is the error obtained by "plugging" the solution to the continuous problem $(E 1)$ into the approximating discrete scheme. In order to do that, note that we can write a system of equations for $\vec{v}$ (defined in (6.16)) in the form

$$
\frac{d}{d t} \vec{v}^{N}(t)=\widetilde{F}^{N}\left(\vec{v}^{N}(t)\right)+N^{2} \widetilde{\Delta}^{N} \vec{v}^{N}(t)+\vec{\varepsilon}^{N}(t),
$$

where $\vec{\varepsilon}^{N}(t):=\left[\vec{\varepsilon}^{N, \alpha}(t), \vec{\varepsilon}^{N, \beta}(t), \vec{\varepsilon}^{N, \gamma}(t)\right]^{T} \in \mathbb{R}^{3 N}$ consists of putting together three vectors in $\mathbb{R}^{N}$ defined as follows: $\vec{\varepsilon}^{N, \alpha} \in \mathbb{R}^{N}$ has components

$$
\varepsilon_{k}^{N, \alpha}(t):=k_{A} \partial_{x}^{2} \alpha(t,(k-1) / N)-k_{A} N^{2}\left[\alpha_{k-1}^{N}(t)-2 \alpha_{k}^{N}(t)+\alpha_{k+1}^{N}(t)\right]
$$

and $\vec{\varepsilon}^{N, \beta}, \vec{\varepsilon}^{N, \gamma}$ are defined similarly.

Thus

$$
\left|\vec{\varepsilon}^{N}(t)\right|_{3 N}^{2}:=\left|\vec{\varepsilon}^{N, \alpha}(t)\right|_{N}^{2}+\left|\vec{\varepsilon}^{N, \beta}(t)\right|_{N}^{2}+\left|\vec{\varepsilon}^{N, \gamma}(t)\right|_{N}^{2}:=\sum_{k=1}^{N}\left|\varepsilon_{k}^{N, \alpha}(t)\right|^{2}+\sum_{k=1}^{N}\left|\varepsilon_{k}^{N, \beta}(t)\right|^{2}+\sum_{k=1}^{N}\left|\varepsilon_{k}^{N, \gamma}(t)\right|^{2}
$$

We see that

$$
\left|\varepsilon_{k}^{N, \alpha}(t)\right| \leq \frac{1}{N}\left\|\partial_{x}^{3} \alpha(t, \cdot)\right\|_{L^{\infty}(0,1)}, \quad\left|\varepsilon_{k}^{N, \beta}(t)\right| \leq \frac{1}{N}\left\|\partial_{x}^{3} \beta(t, \cdot)\right\|_{L^{\infty}(0,1)}, \quad\left|\varepsilon_{k}^{N, \gamma}(t)\right| \leq \frac{1}{N}\left\|\partial_{x}^{3} \gamma(t, \cdot)\right\|_{L^{\infty}(0,1)}
$$

From [14] we know that there exists a unique solution $(\alpha(t, x), \beta(t, x), \gamma(t, x))$ in $[0, \infty) \times I$ for the system $(E 1)$ under essentially bounded initial conditions. By Theorem 6.3 we know that there exists a positive constant $M$ such that

$$
M \geq \alpha \geq 0, \quad M \geq \beta \geq 0, \quad M \geq \gamma \geq 0 \quad \text { in }[0, \infty) \times I .
$$


So, according to (6.23) and (6.24), the boundedness of the third spatial derivatives is sufficient for a consistency estimate.

Theorem 6.5. Let $a_{0}, b_{0}, c_{0} \in L^{\infty}(0,1)$ and $a_{0} \geq 0, b_{0} \geq 0, c_{0} \geq 0$ a.e. in I. Let $k_{A}, k_{B}, k_{C} \geq 0$ and $k_{-1}, k_{1}>0$. Consider the solution $(\alpha, \beta, \gamma)$ for the system $(E 1)$ with initial data $a_{0}, b_{0}, c_{0}$. Fix $0<T<\infty$. Then for any integer $j \geq 1$ and any $0<\delta<T$ the derivatives $\partial_{x}^{j} \alpha, \partial_{x}^{j} \beta, \partial_{x}^{j} \gamma \in L^{\infty}((\delta, T) \times I$ ) (with norm upper bounds depending only on $j$, $\left.\delta, T, M, k_{-1}, k_{1}\right)$.

Proof.

By Duhamel's Principle, we have

$$
\begin{aligned}
\alpha(t, x)= & \int_{I} H\left(k_{A}(t-\delta), x, y\right) \alpha(\delta, y) d y \\
& +\int_{\delta}^{t} \int_{I} H\left(k_{A}(t-s), x, y\right)\left[k_{-1} \gamma(s, y)-k_{1} \alpha(s, y) \beta(s, y)\right] d y d s
\end{aligned}
$$

for $t>\delta \geq 0$. It is easy to see that

$$
\int_{I}\left|\partial_{y} H\left(k_{A} t, x, y\right)\right| d y \leq C\left(k_{A}, \delta, T\right)<\infty \quad \text { for all } \delta \leq t<T, \quad \text { uniformly in } x \in I
$$

Proposition 7.1 also guarantees, in light of the property $\left(5^{\prime}\right)$ for $H$, that, for all $t \in(0, T]$, the kernel $H(t, x, y)$ satisfies (uniformly in $x \in I$ )

$$
\int_{\delta}^{t} \int_{I}\left|\partial_{x} H\left(k_{A}(t-s), x, y\right)\right| d y d s \leq C\left(k_{A}, \delta, T\right)<\infty .
$$

Therefore, we can differentiate under the integral in (6.25) to see that, if $2 \delta \leq t \leq T$, then $\alpha(t, \cdot)$ is differentiable on $(0,1)$, and

$$
\begin{aligned}
\partial_{x} \alpha(t, x)= & \int_{I} \partial_{x} H\left(k_{A}(t-\delta), x, y\right) \alpha(\delta, y) d y \\
& +\int_{\delta}^{t} \int_{I} \partial_{x} H\left(k_{A}(t-s), x, y\right)\left[k_{-1} \gamma(s, y)-k_{1} \alpha(s, y) \beta(s, y)\right] d y d s
\end{aligned}
$$


Now, we use the property $\left(5^{\prime}\right)$ from Appendix and replace $\partial_{x} H$ by $-\partial_{y} H_{D}$, which implies

$$
\begin{aligned}
\partial_{x} \alpha(t, x)= & -\int_{I} \partial_{y} H_{D}\left(k_{A}(t-\delta), x, y\right) \alpha(\delta, y) d y \\
& -\int_{\delta}^{t} \int_{I} \partial_{y} H_{D}\left(k_{A}(t-s), x, y\right)\left[k_{-1} \gamma(s, y)-k_{1} \alpha(s, y) \beta(s, y)\right] d y d s .
\end{aligned}
$$

Therefore, $\alpha(t, \cdot)$ is differentiable on $(0,1)$. The Neumann boundary conditions also give that $\partial_{x} \alpha(t, 0)=\partial_{x} \alpha(t, 1)=0$, so $\alpha(t, \cdot)$ is differentiable on $I$, with zero slopes at boundary.

Obviously, $\beta$ and $\gamma$ enjoy the same regularity. Thus, we can integrate by parts (in space) in $(6.26)$ to get

$$
\begin{aligned}
\partial_{x} \alpha(t, x)=\int_{I} H_{D}\left(k_{A}(t-\delta), x, y\right) \partial_{y} \alpha(\delta, y) d y & +\int_{\delta}^{t} \int_{I} H_{D}\left(k_{A}(t-s), x, y\right)\left[k_{-1} \partial_{y} \gamma(s, y)\right. \\
& \left.-k_{1} \alpha(s, y) \partial_{y} \beta(s, y)-k_{1} \beta(s, y) \partial_{y} \alpha(s, y)\right] d y d s
\end{aligned}
$$

where we used that $H_{D}(t, x, 0)=H_{D}(t, x, 1)=0$ for all $t>0, x \in I$.

Let $u(t, x):=\partial_{x} \alpha(t, x), v(t, x):=\partial_{x} \beta(t, x), w(t, x):=\partial_{x} \gamma(t, x)$. From (6.27), we get

$$
\begin{aligned}
|u(t, x)| \leq\left|\int_{I} H_{D}\left(k_{A}(t-\delta), x, y\right) u(\delta, y) d y\right| & +\int_{\delta}^{t}\left\{k_{-1}\left|\int_{I} H_{D}\left(k_{A}(t-s), x, y\right) w(s, y) d y\right|\right. \\
& +k_{1} M\left|\int_{I} H_{D}\left(k_{A}(t-s), x, y\right) v(s, y) d y\right| \\
& \left.+k_{1} M\left|\int_{I} H_{D}\left(k_{A}(t-s), x, y\right) u(s, y) d y\right|\right\} d s .
\end{aligned}
$$

Since for any $f \in L^{\infty}(I)$, we have that $g(t, x):=\int_{I} H_{D}\left(k_{A} t, x, y\right) f(y) d y$ satisfies $g(0, \cdot)=$ $f$ and solves $\partial_{t} g-k_{A} \partial_{x}^{2} g=0$ with Dirichlet boundary conditions, we conclude that each term whose absolute value is taken in the right hand side of the above inequality is the solution of the Dirichlet problem originating from the indicated function and evaluated at a later time; by property (2) Appendix for $H_{D}$, we conclude

$$
|u(t, x)| \leq\|u(\delta, \cdot)\|_{\infty}+\int_{\delta}^{t}\left\{k_{1} M\left(\|u(s, \cdot)\|_{\infty}+\|v(s, \cdot)\|_{\infty}\right)+k_{-1}\|w(s, \cdot)\|_{\infty}\right\} d s .
$$

Let $\lambda\left(k_{-1}, k_{1}, M\right):=\max \left\{k_{1} M, k_{-1}\right\}$. Then, for all $x \in I$ we have

$$
|u(t, x)| \leq\|u(\delta, \cdot)\|_{\infty}+\lambda\left(k_{-1}, k_{1}, M\right) \int_{\delta}^{t}\left(\|u(s, \cdot)\|_{\infty}+\|v(s, \cdot)\|_{\infty}+\|w(s, \cdot)\|_{\infty}\right) d s
$$


Likewise, we get

$$
|v(t, x)| \leq\|v(\delta, \cdot)\|_{\infty}+\lambda\left(k_{-1}, k_{1}, M\right) \int_{\delta}^{t}\left(\|u(s, \cdot)\|_{\infty}+\|v(s, \cdot)\|_{\infty}+\|w(s, \cdot)\|_{\infty}\right) d s
$$

and

$$
|w(t, x)| \leq\|w(\delta, \cdot)\|_{\infty}+\lambda\left(k_{-1}, k_{1}, M\right) \int_{\delta}^{t}\left(\|u(s, \cdot)\|_{\infty}+\|v(s, \cdot)\|_{\infty}+\|w(s, \cdot)\|_{\infty}\right) d s
$$

By addition and an application of Gronwall's Lemma, we get

$$
\|u(t, \cdot)\|_{\infty}+\|v(t, \cdot)\|_{\infty}+\|w(t, \cdot)\|_{\infty} \leq C_{1}\left(k_{-1}, k_{1}, \delta, T, M\right) \quad \text { for all } t \in[2 \delta, T]
$$

We now return to (6.27) and, using now that the Dirichlet Kernel satisfies (see Appendix)

$$
\int_{I}\left|\partial_{x} H_{D}(t, x, y)\right| d y<\infty \quad \text { for } t \geq 2 \delta, \text { uniformly in } x \in I
$$

and

$$
\int_{\delta}^{t} \int_{I}\left|\partial_{x} H_{D}(t-s, x, y)\right| d y d s \leq C\left(k_{A}, \delta, T\right)<\infty
$$

we conclude that we can differentiate again with respect to $x$ under the integral signs. Therefore, we have

$$
\begin{aligned}
\partial_{x}^{2} \alpha(t, x)= & \int_{I} \partial_{x} H_{D}\left(k_{A}(t-\delta), x, y\right) u(\delta, y) d y \\
& +\int_{\delta}^{t} \int_{I} \partial_{x} H_{D}\left(k_{A}(t-s), x, y\right)\left[k_{-1} w(s, y)\right. \\
& \left.-k_{1} \beta(s, y) u(s, y)-k_{1} \alpha(s, y) v(s, y)\right] d y d s
\end{aligned}
$$

We use the property (5) Appendix for $H$ again to replace $\partial_{x} H_{D}$ by $-\partial_{y} H$. Note that even though $H(t, x, \cdot)$ is not equal to zero at $y=0,1$, we can still integrate by parts and get rid of the boundary terms because $u(s, \cdot), v(s, \cdot), w(s, \cdot)$ are all zero at $y=0,1$ for all $s \in[\delta, t]$. 
Therefore, we get

$$
\begin{array}{r}
\partial_{x}^{2} \alpha(t, x)=\int_{I} H\left(k_{A}(t-\delta), x, y\right) \partial_{y} u(\delta, y) d y+\int_{\delta}^{t} \int_{I} H\left(k_{A}(t-s), x, y\right)\left[k_{-1} \partial_{y} w(s, y)\right. \\
\left.-2 k_{1} u(s, y) v(s, y)-k_{1} \beta(s, y) \partial_{y} u(s, y)-k_{1} \alpha(s, y) \partial_{y} v(s, y)\right] d y d s
\end{array}
$$

We use the property $\left(2^{\prime}\right)$ Appendix for $H$ to conclude

$$
\begin{aligned}
\left\|\partial_{x}^{2} \alpha(t, \cdot)\right\|_{\infty} & =\sup _{x \in I}\left|\partial_{x}^{2} \alpha(t, x)\right| \\
\leq & \left\|\partial_{y}^{2} \alpha(\delta, \cdot)\right\|_{\infty}+\int_{\delta}^{t}\left\{\begin{array}{r}
k_{-1} \\
\left\|\partial_{y}^{2} \gamma(s, \cdot)\right\|_{\infty}+2 k_{1}\|u(s, \cdot)\|_{\infty}\|v(s, \cdot)\|_{\infty} \\
\left.\quad+k_{1} M\left\|\partial_{y}^{2} \alpha(s, \cdot)\right\|_{\infty}+k_{1} M\left\|\partial_{y}^{2} \beta(s, \cdot)\right\|_{\infty}\right\} d s .
\end{array}\right.
\end{aligned}
$$

We use (6.28) to bound the term $\|u(s, \cdot)\|_{\infty}\|v(s, \cdot)\|_{\infty}$, write the corresponding inequalities for the $\beta$ and $\gamma$ terms, add them up and use Gronwall's Lemma again to get a bound $C_{2}\left(k_{-1}, k_{1}, \delta, T, M\right)<\infty$ on $\left\|\partial_{x}^{2} \alpha(t, \cdot)\right\|_{\infty}+\left\|\partial_{x}^{2} \beta(t, \cdot)\right\|_{\infty}+\left\|\partial_{x}^{2} \gamma(t, \cdot)\right\|_{\infty}$ for $t \in[2 \delta, T]$.

From (6.29), we differentiate again in $x$ to get (after using the property $\left(5^{\prime}\right)$ for $H$ yet again)

$$
\begin{gathered}
\partial_{x}^{3} \alpha(t, x)=-\int_{I} \partial_{y} H_{D}\left(k_{A}(t-\delta), x, y\right) \partial_{y}^{2} \alpha(\delta, y) d y-\int_{\delta}^{t} \int_{I} \partial_{y} H_{D}\left(k_{A}(t-s), x, y\right)\left[k_{-1} \partial_{y}^{2} \gamma(s, y)\right. \\
\left.-2 k_{1} \partial_{y} \alpha(s, y) \partial_{y} \beta(s, y)-k_{1} \beta(s, y) \partial_{y}^{2} \alpha(s, y)-k_{1} \alpha(s, y) \partial_{y}^{2} \beta(s, y)\right] d y d s .
\end{gathered}
$$

This time we deal with the Dirichlet Kernel once more, so even if $\partial_{y}^{2} \alpha(\delta, y)$ and the likes do not vanish at $y=0,1, H_{D}(t, x, \cdot)$ does for all $t>0$ and all $x \in I$. Therefore, we can once more integrate by parts to get

$$
\begin{aligned}
\partial_{x}^{3} \alpha(t, x)= & \int_{I} H_{D}\left(k_{A}(t-\delta), x, y\right) \partial_{y}^{3} \alpha(\delta, y) d y+\int_{\delta}^{t} \int_{I} H_{D}\left(k_{A}(t-s), x, y\right)\left[k_{-1} \partial_{y}^{3} \gamma(s, y)\right. \\
& -3 k_{1} \partial_{y}^{2} \alpha(s, y) \partial_{y} \beta(s, y)-3 k_{1} \partial_{y} \alpha(s, y) \partial_{y}^{2} \beta(s, y) \\
& \left.-k_{1} \beta(s, y) \partial_{y}^{3} \alpha(s, y)-k_{1} \alpha(s, y) \partial_{y}^{3} \beta(s, y)\right] d y d s
\end{aligned}
$$

which implies

$$
\begin{aligned}
& \left|\partial_{x}^{3} \alpha(t, x)\right| \leq\left\|\partial_{y}^{3} \alpha(\delta, \cdot)\right\|_{\infty}+\int_{\delta}^{t}\left\{k_{-1}\left\|\partial_{y}^{3} \gamma(s, \cdot)\right\|_{\infty}+\widetilde{C}\left(k_{-1}, k_{1}, M, T, \delta\right)\right. \\
& \left.+k_{1} M\left\|\partial_{y}^{3} \alpha(s, \cdot)\right\|_{\infty}+k_{1} M\left\|\partial_{y}^{3} \beta(s, \cdot)\right\|_{\infty}\right\} d s .
\end{aligned}
$$


Again, by Gronwall's Lemma, we get

$$
\left\|\partial_{x}^{3} \alpha(t, \cdot)\right\|_{\infty}+\left\|\partial_{x}^{3} \beta(t, \cdot)\right\|_{\infty}+\left\|\partial_{x}^{3} \gamma(t, \cdot)\right\|_{\infty} \leq C_{3}\left(k_{-1}, k_{1}, M, T, \delta\right)<\infty
$$

The procedure can be continued to get bounds of the type

$$
\left\|\partial_{x}^{j} \alpha(t, \cdot)\right\|_{\infty}+\left\|\partial_{x}^{j} \beta(t, \cdot)\right\|_{\infty}+\left\|\partial_{x}^{j} \gamma(t, \cdot)\right\|_{\infty} \leq C\left(j, k_{-1}, k_{1}, M, T, \delta\right)<\infty
$$

for all orders of differentiation $j \geq 1$.

By (6.23) and (6.24) we get that

$$
\left|\vec{\varepsilon}^{N}(t)\right|_{3 N}^{2} \leq \frac{1}{N} C^{2}\left(k_{-1}, k_{1}, M, T, \delta\right)
$$

Thus, we have:

Theorem 6.6. For any $0<\delta<T<\infty$ there exists a real constant $C(\delta, T)$ such that

$$
\left|\vec{\varepsilon}^{N}(t)\right|_{3 N}^{2} \leq \frac{1}{N} C(\delta, T) \text { for all integers } N \geq 2 \text { and all } t \in[\delta, T]
$$

proof. Of course, since $k_{-1}, k_{1}, M$ are fixed here, we can denote $C^{2}\left(k_{-1}, k_{1}, M, T, \delta\right)$ by $C(\delta, T)$ and use (6.31) to conclude.

\subsubsection{Convergence}

Let $T>0$ and assume that the initial data $a_{0}, b_{0}$ and $c_{0}$ in $L^{\infty}(0,1)$. Then, by Theorem 6.4 there exists a finite number $\tilde{M}$ such that

$0 \leq a_{k}^{N}(t) \leq \tilde{M}, \quad 0 \leq b_{k}^{N}(t) \leq \tilde{M}, \quad 0 \leq c_{k}^{N}(t) \leq \tilde{M} \quad$ for all $N, k=1, \ldots, N$, and $0 \leq t \leq T$.

In fact, $\tilde{M}$ is inside of the interval $I$.

Let $a_{0}, b_{0}, c_{0} \in L^{\infty}(0,1)$ and define, for all integers $N \geq 1$, the vectors $\vec{a}_{0}^{N}, \vec{b}_{0}^{N}, \vec{c}_{0}^{N} \in \mathbb{R}^{n}$ 
whose components are given by

$$
\begin{aligned}
a_{0, k}^{N}(x) & :=N \int_{(k-1) / N}^{k / N} a_{0}(x) d x \text { for } k=1, \ldots, N, \\
b_{0, k}^{N}(x) & :=N \int_{(k-1) / N}^{k / N} b_{0}(x) d x \text { for } k=1, \ldots, N, \\
c_{0, k}^{N}(x) & :=N \int_{(k-1) / N}^{k / N} c_{0}(x) d x \text { for } k=1, \ldots, N .
\end{aligned}
$$

Note that all these lie in the interval $[0, \tilde{M}]$.

\section{Proof of the main theorem}

Proof of Theorem 6.1: Let us notice that (6.3) is trivial for $t=0$ (see Appendix, Subsection 7.1.5). For $t \in(0, T)$ the proof is presented in three steps: first we prove that

$$
\lim _{N \rightarrow \infty}\left[\left\|\alpha(t, \cdot)-\alpha^{N}(t, \cdot)\right\|_{2}+\left\|\beta(t, \cdot)-\beta^{N}(t, \cdot)\right\|_{2}+\left\|\gamma(t, \cdot)-\gamma^{N}(t, \cdot)\right\|_{2}\right]=0
$$

This is a straightforward consequence of Theorem 6.5 , Section 6.2 for $j=1$. Indeed, since

$$
\left\|\alpha(t, \cdot)-\alpha^{N}(t, \cdot)\right\|_{2}^{2}=\sum_{k=1}^{N} \int_{(k-1) / N}^{k / N}|\alpha(t, x)-\alpha(t,(k-1) / N)|^{2} d x,
$$

the bound on $\partial_{x} \alpha(t, \cdot)$ provided by Theorem 6.5 shows that this quantity tends to vanish as $N \rightarrow \infty$. The same is, obviously, true about the $\beta$ and $\gamma$ terms. Thus, (6.3) would follow from

$$
\lim _{N \rightarrow \infty}\left[\left\|a^{N}(t, \cdot)-\alpha^{N}(t, \cdot)\right\|_{2}+\left\|b^{N}(t, \cdot)-\beta^{N}(t, \cdot)\right\|_{2}+\left\|c^{N}(t, \cdot)-\gamma^{N}(t, \cdot)\right\|_{2}\right]=0 .
$$

Next, let us prove (6.33). Let us denote by $\langle\cdot, \cdot\rangle_{N}$ the Euclidean inner product on $\mathbb{R}^{N}$ for any positive integer $N$, and by $|\cdot|_{N}$ the associated norm, i.e.

$$
\langle\vec{u}, \vec{v}\rangle_{N}=\sum_{i=1}^{N} u_{i} v_{i} \quad \text { for all } \vec{u}, \vec{v} \in \mathbb{R}^{N}
$$


We denote by $\|\cdot\|$ the $L^{2}(0,1)$ norm, and denote (we give three equivalent expressions)

$$
\begin{aligned}
e^{N}(t) & :=\frac{1}{2}\left[\left\|a^{N}(t, \cdot)-\alpha^{N}(t, \cdot)\right\|^{2}+\left\|b^{N}(t, \cdot)-\beta^{N}(t, \cdot)\right\|^{2}+\left\|c^{N}(t, \cdot)-\gamma^{N}(t, \cdot)\right\|^{2}\right] \\
& =\frac{1}{2 N}\left[\left|\vec{a}^{N}(t)-\vec{\alpha}^{N}(t)\right|_{N}^{2}+\left|\vec{b}^{N}(t)-\vec{\beta}^{N}(t)\right|_{N}^{2}+\left|\vec{c}^{N}(t)-\vec{\gamma}^{N}(t)\right|_{N}^{2}\right] \\
& =\frac{1}{2 N}\left|\vec{u}^{N}(t)-\vec{v}^{N}(t)\right|_{3 N}^{2} .
\end{aligned}
$$

Take the time derivative to see that

$$
\dot{e}^{N}(t)=\frac{1}{N}\left\langle\vec{u}^{N}(t)-\vec{v}^{N}(t), \frac{d}{d t} \vec{u}^{N}(t)-\frac{d}{d t} \vec{v}^{N}(t)\right\rangle_{3 N} .
$$

From (6.18), (6.22) and (6.34) we obtain

$$
\begin{aligned}
\dot{e}^{N}(t)=\frac{1}{N} & \left\langle\vec{u}^{N}(t)-\vec{v}^{N}(t), \widetilde{F}^{N}\left(\vec{u}^{N}(t)\right)-\widetilde{F}^{N}\left(\vec{v}^{N}(t)\right)\right\rangle_{3 N} \\
& +\frac{1}{N}\left\langle\vec{u}^{N}(t)-\vec{v}^{N}(t), \tilde{\Delta}^{N}\left[\vec{u}^{N}(t)-\vec{v}^{N}(t)\right]\right\rangle_{3 N} \\
& -\frac{1}{N}\left\langle\vec{u}^{N}(t)-\vec{v}^{N}(t), \vec{\varepsilon}^{N}(t)\right\rangle_{3 N} .
\end{aligned}
$$

For the first term in the right hand side of the above display we use the Mean Value Theorem for vector fields to write

$$
\widetilde{F}^{N}\left(\vec{u}^{N}(t)\right)-\widetilde{F}^{N}\left(\vec{v}^{N}(t)\right)=\int_{I} \operatorname{Jac}\left(\widetilde{F}^{N}\left((1-\theta) \vec{u}^{N}(t)+\theta \vec{v}^{N}(t)\right)\right) d \theta \cdot\left(\vec{u}^{N}(t)-\vec{v}^{N}(t)\right)
$$

First we compute Jac (the Jacobian) for $\widetilde{F}^{N}$. We set $\vec{y}(t):=\vec{u}^{N}(t)-\vec{v}^{N}(t) \in \mathbb{R}^{3 N}$, where $\vec{y}(t)=\left[y_{1}(t), \ldots, y_{N}(t), y_{N+1}(t), \ldots, y_{2 N}(t), y_{2 N+1}(t), \ldots, y_{3 N}\right]^{T}$. Then, we have

$$
\operatorname{Jac}\left(\widetilde{F}^{N}\left((1-\theta) \vec{u}^{N}(t)+\theta \vec{v}^{N}(t)\right)\right)=\left[\begin{array}{ccc}
\tilde{B} & \tilde{A} & \tilde{C} \\
\tilde{B} & \tilde{A} & \tilde{C} \\
-\tilde{B} & -\tilde{A} & -\tilde{C}
\end{array}\right]_{3 N \times 3 N}
$$

where $\tilde{A}=-k_{1} \operatorname{diag}\left[\vec{a}^{N}(t)\right]_{N \times N}, \tilde{B}=-k_{1} \operatorname{diag}\left[\vec{b}^{N}(t)\right]_{N \times N}$, and $\tilde{C}=k_{-1} I_{N \times N}$; here $I$ denotes the identity matrix. 
Multiply both sides of $(6.35)$ by $\vec{y}(t)$ to get

$$
\vec{y}(t) \cdot\left[\widetilde{F}^{N}\left(\vec{u}^{N}\right)-\widetilde{F}^{N}\left(\vec{v}^{N}\right)\right]=\left(\int_{I} \operatorname{Jac}\left(\widetilde{F}^{N}\left((1-\theta) \vec{u}^{N}(t)+\theta \vec{v}^{N}(t)\right)\right) d \theta \vec{y}(t)\right) \cdot \vec{y}(t) .
$$

But

$$
\begin{aligned}
& \vec{y}(t) \cdot \operatorname{Jac}\left(\widetilde{F}^{N}\left((1-\theta) \vec{u}^{N}(t)+\theta \vec{v}^{N}(t)\right)\right) \vec{y}(t)=-k_{1}\left(b_{1}^{N}(t) y_{1}^{2}+a_{1}^{N}(t) y_{1} y_{N}\right)+k_{-1} y_{1} y_{2 N+1} \\
& +\cdots-k_{1}\left(b_{N}^{N}(t) y_{N}^{2}+a_{N}^{N}(t) y_{N} y_{2 N}\right)+k_{-1} y_{N} y_{3 N}-k_{1}\left(b_{1}^{N}(t) y_{1} y_{N}+a_{1}^{N}(t) y_{N}^{2}\right)+k_{-1} y_{N} y_{2 N+1} \\
& +\ldots-k_{1}\left(b_{N}^{N}(t) y_{N} y_{2 N}+a_{N}^{N}(t) y_{2 N}^{2}\right)+k_{-1} y_{2 N} y_{3 N}+k_{1}\left(b_{1}^{N}(t) y_{1} y_{2 N}+a_{1}^{N}(t) y_{2 N} y_{N}\right)-k_{-1} y_{2 N+1}^{2} \\
& +\ldots+k_{1}\left(b_{N}^{N}(t) y_{N} y_{3 N}+a_{N}^{N}(t) y_{2 N} y_{3 N}\right)-k_{-1} y_{3 N}^{2}
\end{aligned}
$$

and so, since all the $a_{k}^{N}$ 's, $b_{k}^{N}$ 's, etc lie in the interval $[0, \tilde{M}]$ for some $0<\tilde{M}<\infty$ for all $N, k$, we have

$$
\begin{aligned}
& \vec{y}(t) \cdot \operatorname{Jac}\left(\widetilde{F}^{N}\left((1-\theta) \vec{u}^{N}(t)+\theta \vec{v}^{N}(t)\right)\right) \vec{y}(t) \leq k_{-1} y_{1} y_{2 N+1}+\cdots+k_{-1} y_{N} y_{3 N}+k_{-1} y_{N} y_{2 N+1} \\
& +\cdots+k_{-1} y_{2 N} y_{3 N}+k_{1}\left(b_{1}^{N}(t) y_{1} y_{2 N+1}+a_{1}^{N}(t) y_{2 N+1} y_{N}\right)+\cdots+k_{1}\left(b_{N}^{N}(t) y_{N} y_{3 N}+a_{N}^{N}(t) y_{2 N} y_{3 N}\right) \\
& =\left(k_{-1}+k_{1} b_{1}^{N}(t)\right) y_{1} y_{2 N+1}+\cdots+\left(k_{-1}+k_{1} b_{N}^{N}(t)\right) y_{N-1} y_{3 N}+\left(k_{-1}+k_{1} a_{1}^{N}(t)\right) y_{2 N+1} y_{N} \\
& +\cdots+\left(k_{-1}+k_{1} a_{N 1}^{N}(t)\right) y_{2 N} y_{3 N} \\
& \leq \frac{\left(k_{-1}+k_{1} \tilde{M}\right)}{2}\left(y_{1}^{2}+\cdots+y_{2 N}^{2}+2 y_{2 N+1}^{2}+\cdots+2 y_{3 N}^{2}\right) .
\end{aligned}
$$

From (6.37) we conclude that

$$
\vec{y} \cdot \operatorname{Jac}\left(\widetilde{F}^{N}\left((1-\theta) \vec{u}^{N}(t)+\theta \vec{v}^{N}(t)\right)\right) \vec{y} \leq\left(k_{-1}+k_{1} \tilde{M}\right)|y|_{3 N}^{2}
$$

Thus,

$$
\frac{1}{N}\left\langle\vec{u}^{N}(t)-\vec{v}^{N}(t), \widetilde{F}^{N}\left(\vec{u}^{N}(t)\right)-\widetilde{F}^{N}\left(\vec{v}^{N}(t)\right)\right\rangle_{3 N} \leq C(\tilde{M}) e^{N}(t),
$$

where $C(\tilde{M})=2\left(k_{-1}+k_{1} \tilde{M}\right) \in \mathbb{R}$ is independent of $N, \delta$ and $T$.

The term in the middle is nonpositive because $-\tilde{\Delta}^{N}$ is a nonnegative-definite matrix. Finally, 
in light of the Cauchy-Schwarz inequality and (6.30), the last term is bounded above by

$$
e^{N}(t)+\frac{1}{2 N}\left|\vec{\varepsilon}^{N}(t)\right|_{3 N}^{2} \leq e^{N}(t)+\frac{3 C(\delta, T)}{2 N^{2}} .
$$

Thus, we have

$$
\dot{e}^{N}(t) \leq(C(\tilde{M})+1) e^{N}(t)+\frac{3 C(\delta, T)}{2 N^{2}} \quad \text { for all } \delta \leq t \leq T
$$

which implies

$$
\dot{e}^{N}(t) \leq C_{1} e^{N}(t)+E(N)
$$

for some constant $C_{1}=(C(\tilde{M})+1)$ which is independent of $t, N$ and $E(N):=\frac{3 C(\delta, T)}{2 N^{2}}$. Then

$$
\frac{d}{d t}\left(\exp \left(-C_{1} t\right) e^{N}(t)\right) \leq E(N) \exp \left(-C_{1} t\right)
$$

Fix $0<t<T$ for given $T>0$, and let $\delta \in(0, t)$. Integrate (6.39) from $\delta$ to $t$ to get

$$
e^{N}(t) \leq \exp \left(C_{1}(t-\delta)\right) e^{N}(\delta)+\frac{E(N)}{C_{1}}\left[\exp \left(C_{1}(t-\delta)\right)-1\right]
$$

and then let $N$ go to infinity to conclude

$$
\limsup _{N \rightarrow \infty} e^{N}(t) \leq \exp \left(C_{1}(t-\delta)\right) \limsup _{N \rightarrow \infty} e^{N}(\delta) \quad \text { for all } t \in[\delta, T]
$$

Finally, let $\delta \rightarrow 0^{+}$to obtain

$$
\limsup _{N \rightarrow \infty} e^{N}(t) \leq C(T) \liminf _{\delta \rightarrow 0^{+}} \limsup _{N \rightarrow \infty} e^{N}(\delta),
$$

where $C(T)=\exp \left(C_{1} T\right)$.

\section{Claim :}

$$
\liminf _{\delta \rightarrow 0^{+}} \limsup _{N \rightarrow \infty} e^{N}(\delta)=0 .
$$

Observation: If the statement above holds, we deduce

$$
\limsup _{N \rightarrow \infty} e^{N}(t)=0
$$


which proves convergence of the discrete problem solution to the continuous problem solution.

Proof of Claim: Since

$$
\alpha(\delta, x)=\int_{I} H_{1}(\delta, x, y) a_{0}(y) d y+\int_{0}^{\delta} \int_{I} H_{1}(s, x, y) f_{1}(\delta-s, y) d y d s
$$

we have

$$
\alpha(\delta, k h)=\int_{I} H_{1}(\delta, k h, y) a_{0}(y) d y+\int_{0}^{\delta} \int_{I} H_{1}(s, k h, y) f_{1}(\delta-s, y) d y d s
$$

By using the discrete Heat Kernel $H_{1}^{N}$, we have

$$
a^{N}(\delta, k h)=\int_{I} H_{1}^{N}(\delta, k h, y) a_{0}^{N}(y) d y+\int_{0}^{\delta} \int_{I} H_{1}^{N}(s, k h, y) f_{1}^{N}(\delta-s, y) d y d s
$$

where $a_{0}^{N}(y):=\int_{(k-1) h_{N}}^{k h_{N}} a_{0}(u) d u$ if $(k-1) h_{N} \leq y<k h_{N}$, and $f_{1}^{N}(s, y):=-k_{1} a^{N}(s, y) b^{N}(s, y)$ $+k_{-1} c^{N}(s, y)$. Note that,

$$
\begin{aligned}
e^{N}(\delta)=\frac{1}{2 N} \sum_{k=1}^{N}\left|\alpha(\delta, k h)-a^{N}(\delta, k h)\right|^{2} & +\frac{1}{2 N} \sum_{k=1}^{N}\left|\beta(\delta, k h)-b^{N}(\delta, k h)\right|^{2} \\
& +\frac{1}{2 N} \sum_{k=1}^{N}\left|\gamma(\delta, k h)-c^{N}(\delta, k h)\right|^{2} .
\end{aligned}
$$

Therefore, let us fix $k \in\{1, \ldots, N\}$ and estimate

$$
\begin{aligned}
\left|\alpha(\delta, k h)-a^{N}(\delta, k h)\right| \leq \mid \int_{I} H_{1}(\delta, k h, y) & a_{0}(y) d y-\int_{I} H_{1}^{N}(\delta, k h, y) a_{0}^{N}(y) d y \mid \\
& +\left(k_{1} M^{2}+K_{-1} M\right) \int_{0}^{\delta} \int_{I}\left|H_{1}(s, k h, y)\right| d y d s \\
& +\left(k_{1} \tilde{M}^{2}+K_{-1} \tilde{M}\right) \int_{0}^{\delta} \int_{I}\left|H_{1}^{N}(s, k h, y)\right| d y d s .
\end{aligned}
$$

Note that, $H_{1}$ is known to be nonnegative and to integrate to 1 in each spatial variable, SO

$$
\int_{I}\left|H_{1}(\delta, k h, y)\right| d y=1 \quad \text { for all } k=1, \ldots, N
$$


We will assume, for simplicity, that $k_{A}=1$; so that we have $H_{1}=H$ and $H_{1}^{N}=H^{N}$. Then

$$
\begin{aligned}
\int_{0}^{\delta} \int_{I}\left|H^{N}(s, k h, y)\right| d y d s & \leq \int_{0}^{\delta}[1+2 f(4 s)] d s \\
& =\delta+\frac{1}{2} \int_{0}^{4 \delta} f(s) d s .
\end{aligned}
$$

Since $f(t)=\sum_{k=1}^{\infty} e^{-k^{2} t}$ is positive and integrable on $(0, \infty)$ (see Appendix, Subsection 7.1.4), we have

$$
\lim _{\delta \rightarrow 0^{+}}\left[\delta+\frac{1}{2} \int_{0}^{4 \delta} f(s) d s\right]=0
$$

Therefore, we have bounds on the last two terms in the right hand side of (6.40), bounds which only depend on $\delta$ (and not on $k, N$ ) and tend to 0 as $\delta \rightarrow 0^{+}$.

Next, let us check the first term in the right hand side of (6.40) (call it T1). We have

$$
T 1 \leq \int_{I} H(\delta, k h, y)\left|a_{0}(y)-a_{0}^{N}(y)\right| d y+\int_{I}\left|H(\delta, k h, y)-H^{N}(\delta, k h, y)\right| a_{0}^{N}(y) d y
$$

By Appendix (7.18) we have $H(\delta, k h, y) \leq 1+2 f(4 \delta)=: C(\delta)$ for all $y \in I$. Since $a_{0}^{N}$ converges in $L^{1}(I)$ to $a_{0}$, we may take $N$ sufficiently large so that $\left\|a_{0}-a_{0}^{N}\right\|_{L^{1}(0,1)} \leq \delta / C(\delta)$ (see Appendix (7.19)). Also, $\left|a_{0}^{N}(y)\right| \leq \tilde{M}$ for all $N, y$. Therefore, we get

$$
\begin{aligned}
T 1 & \leq \delta \int_{I} H(\delta, k h, y) d y+\tilde{M} \int_{I}\left|H(\delta, k h, y)-H^{N}(\delta, k h, y)\right| d y \\
& =\delta+\tilde{M} \int_{I}\left|H(\delta, k h, y)-H^{N}(\delta, k h, y)\right| d y
\end{aligned}
$$

if $N$ is sufficiently large.

But for all $t>0, H^{N}(t, \cdot, \cdot)$ converges uniformly to $H(t, \cdot, \cdot)$ (see Appendix). Therefore, we have

$$
\tilde{M} \int_{I}\left|H(\delta, k h, y)-H^{N}(\delta, k h, y)\right| d y \leq \delta
$$

if $N$ is sufficiently large. Therefore,

$$
\left|\alpha(\delta, k h)-a^{N}(\delta, k h)\right| \leq 2 \delta+\delta\left(k_{1} M^{2}+k_{-1} M\right)+\left(k_{1} \tilde{M}^{2}+k_{-1} \tilde{M}\right)\left[\delta+\frac{1}{2} \int_{0}^{4 \delta} f(s) d s\right]
$$

Similarly, we have

$$
\left|\beta(\delta, k h)-b^{N}(\delta, k h)\right| \leq 2 \delta+\delta\left(k_{1} M^{2}+k_{-1} M\right)+\left(k_{1} \tilde{M}^{2}+k_{-1} \tilde{M}\right)\left[\delta+\frac{1}{2} \int_{0}^{4 \delta} f(s) d s\right]
$$




$$
\left|\gamma(\delta, k h)-c^{N}(\delta, k h)\right| \leq 2 \delta+\delta\left(k_{1} M^{2}+k_{-1} M\right)+\left(k_{1} \tilde{M}^{2}+k_{-1} \tilde{M}\right)\left[\delta+\frac{1}{2} \int_{0}^{4 \delta} f(s) d s\right]
$$

In conclusion, for sufficiently large $N$ we have

$$
e^{N}(\delta) \leq 3\left(2 \delta+\delta\left(k_{1} M^{2}+k_{-1} M\right)+\left(k_{1} \tilde{M}^{2}+k_{-1} \tilde{M}\right)\left[\delta+\frac{1}{2} \int_{0}^{4 \delta} f(s) d s\right]\right)^{2}=: O(\delta)
$$

which implies

$$
\liminf _{\delta \rightarrow 0^{+}} \limsup _{N \rightarrow \infty} e^{N}(\delta) \leq \liminf _{\delta \rightarrow 0^{+}} O(\delta)=\lim _{\delta \rightarrow 0^{+}} O(\delta)=0 .
$$

This concludes our proof. 


\section{Chapter 7}

\section{Appendix}

\subsection{Appendix}

\subsubsection{Dirichlet Heat Kernel/Neumann Heat Kernel}

(i) Let $I:=(0,1)$. Then, $H_{D}:(0, \infty) \times I \times I \rightarrow \mathbb{R}$ given by

$$
H_{D}(t, x, y):=2 \sum_{j=1}^{\infty} e^{-j^{2} \pi^{2} t} \sin (j \pi x) \sin (j \pi y)
$$

is the Dirichlet Heat Kernel associated to $I$; that is, for any $u_{0} \in L^{\infty}(I)$, the function $u_{0}:(0, \infty) \times I \rightarrow \mathbb{R}$ given by

$$
u(t, x)=\int_{I} H_{D}(t, x, y) u_{0}(y) d y
$$

is the unique solution to

$$
\begin{cases}\partial_{t} u=\partial_{x}^{2} u & \text { in }(0, \infty) \times I \\ u(\cdot, 0)=u(\cdot, 1)=0 & \text { in }(0, \infty) \\ u(0, \cdot)=u_{0}(\cdot) & \text { in } I .\end{cases}
$$

Properties of $H_{D}$ :

(1) $u(t, x)=\int_{I} H_{D}(t-\delta, x, y) u(\delta, y) d y$ for every $t>\delta \geq 0$. 
(2) Maximum Principle:

$$
\max _{\substack{t \in[0, \infty) \\ x \in I}} u(t, x) \leq \max _{I} u_{0}
$$

(3) We have $H_{D}>0$ in $(0, \infty) \times I \times I$, and

$$
\int_{I} H_{D}(t, x, z) d z \leq 1, \quad \int_{I} H_{D}(t, z, y) d z \leq 1 \quad \text { for all } x, y \in I
$$

(4) It is known that (see, e.g., [17]), there exists a positive constant $C_{D}>0$ such that

$$
H_{D}(t, x, y) \leq \frac{C_{D}}{\sqrt{t}} \exp \left\{-\frac{(x-y)^{2}}{8 t}\right\} \quad \text { for all } t>0, x, y \in I
$$

Observation: Note that $-u$ is also the solution for the initial data $-u_{0}$. So, by the maximum principle, we also have

$$
\min _{\substack{t \in[0,+\infty) \\ x \in I}} u(t, x) \geq \min _{I} u_{0}
$$

So, in general, we have

$$
\|u(t, \cdot)\|_{\infty} \leq\|u(\delta, \cdot)\|_{\infty} \quad \text { for all } t>\delta \geq 0
$$

(ii) Let $H:(0, \infty) \times I \times I \rightarrow \mathbb{R}$ given by

$$
H(t, x, y):=1+2 \sum_{j=1}^{\infty} e^{-j^{2} \pi^{2} t} \cos (j \pi x) \cos (j \pi y)
$$

This is the Neumann Heat Kernel associated to $I$; that is, the function (for any given $\left.u_{0} \in L^{\infty}(I)\right)$

$$
u(t, x)=\int_{I} H(t, x, y) u_{0}(y) d y
$$


is the unique solution to

$$
\begin{cases}\partial_{t} u=\partial_{x}^{2} u & \text { in }(0, \infty) \times I \\ \partial_{x} u(\cdot, 0)=\partial_{x} u(\cdot, 1)=0 & \text { in }(0, \infty) \\ u(0, \cdot)=u_{0}(\cdot) & \text { in } I .\end{cases}
$$

\section{Properties of $H$ :}

$\left(1^{\prime}\right) u(t, x)=\int_{I} H(t-\delta, x, y) u(\delta, y) d y$ for all $t>\delta \geq 0$.

$\left(2^{\prime}\right) H \geq 0$ on its domain and

$$
\int_{I} H(t, x, y) d y=\int_{I} H(t, x, y) d x=1 \quad \text { for all } x, y \in I, \text { and all } t>0
$$

$\left(3^{\prime}\right)$ From $\left(1^{\prime}\right)$ and $\left(2^{\prime}\right)$, we also get

$$
\|u(t, \cdot)\|_{\infty} \leq\|u(\delta, \cdot)\|_{\infty} \quad \text { for all } t>\delta \geq 0
$$

$\left(4^{\prime}\right)$ It is known that (see, e.g., [15]), there exists a positive constant $C_{N}>0$ such that

$$
H(t, x, y) \leq \frac{C_{N}}{\sqrt{t}} \exp \left\{-\frac{(x-y)^{2}}{8 t}\right\} \quad \text { for all } t>0 \text {, and all } x, y \in I \text {. }
$$

$\left(5^{\prime}\right) \partial_{x} H(t, x, y)+\partial_{y} H_{D}(t, x, y)=\partial_{y} H(t, x, y)+\partial_{x} H_{D}(t, x, y)=0$ for all $x, y \in$ $I$, and $t>0$.

Proposition 7.1. There exists a positive real number $C$ such that

$$
\int_{I}\left|\partial_{y} \tilde{H}(t, x, y)\right| d y \leq C t^{-3 / 4} \quad \text { for all }(t, x) \in(0, \infty) \times I,
$$

where $\tilde{H}$ is either $H_{D}$ or $H$.

proof. In the proof of Theorem 1.1 [28], the author shows that if $H_{D}$ satisfies (on some bounded and open subset $\Omega$ of a smooth, connected, complete noncompact Riemannian 
manifold $\mathcal{M})$, for every $(t, x) \in(0, T) \times I$, that

$$
E_{0}(t, x):=\int_{\Omega}\left|H_{D}(t, x, y)\right|^{2} e^{\frac{(x-y)^{2}}{4 t}} d y \leq \frac{1}{f(t)}
$$

for some $0<T<\infty$ and some positive $f \in L^{1}(0, T)$, then

$$
E_{1}(t, x):=\int_{\Omega}\left|\partial_{y} H_{D}(t, x, y)\right|^{2} e^{\frac{(x-y)^{2}}{4 t}} d y \leq \frac{5}{F(t)} \quad \text { for all }(t, x) \in(0, T) \times I,
$$

where $F(t):=\int_{0}^{t} f(s) d s$. From (4) of the above properties for $H_{D}$, we see that if we take $\mathcal{M}=\mathbb{R}$ and $\Omega=I$, then we have the desired bound on $E_{0}$ with $f(t)=t / C_{D}^{2}$. We deduce

$$
\int_{I}\left|\partial_{y} H_{D}(t, x, y)\right|^{2} e^{\frac{(x-y)^{2}}{4 t}} d y \leq \frac{10 C_{D}^{2}}{t^{2}} \quad \text { for all }(t, x) \in(0, T) \times I,
$$

which, by Cauchy-Schwarz, yields

$$
\left(\int_{I}\left|\partial_{y} H_{D}(t, x, y)\right| d y\right)^{2} \leq \frac{10 C_{D}^{2}}{t^{2}} \int_{I} e^{-\frac{(x-y)^{2}}{4 t}} d y \leq 20 \sqrt{\pi} C_{D}^{2} t^{-3 / 2}
$$

So, in the case $H=H_{D}$, the statement is proved for $C:=\sqrt{20 \sqrt{\pi}} C_{D}$. A careful inspection of the proof of Theorem 1.1 [28] reveals that the same argument works for the Neumann Heat Kernel, so, in light of the property $\left(4^{\prime}\right)$ above, we get the desired bound in this case as well.

Recall that the solution $(\alpha, \beta, \gamma)$ of $(E 1)$ with Neumann BC satisfies

$$
\begin{aligned}
\alpha(t, x) & =\int_{I} H(t, x, y) a_{0}(y) d y+\int_{0}^{t} \int_{I} H(t-s, x, y)\left[k_{-1} \gamma(s, y)-k_{1} \alpha(s, y) \beta(s, y)\right] d y d s, \\
\beta(t, x) & =\int_{I} H(t, x, y) b_{0}(y) d y+\int_{0}^{t} \int_{I} H(t-s, x, y)\left[k_{-1} \gamma(s, y)-k_{1} \alpha(s, y) \beta(s, y)\right] d y d s, \\
\gamma(t, x) & =\int_{I} H(t, x, y) c_{0}(y) d y+\int_{0}^{t} \int_{I} H(t-s, x, y)\left[-k_{-1} \gamma(s, y)+k_{1} \alpha(s, y) \beta(s, y)\right] d y d s .
\end{aligned}
$$

Let us now mimic this representation formula in the discrete case below. 


\subsubsection{The Neumann Heat Kernel associated to the discrete case}

Now, we solve the system

$$
\dot{u}_{k}^{N}(t)=\frac{1}{h_{N}^{2}}\left(u_{k-1}^{N}(t)-2 u_{k}^{N}(t)+u_{k+1}^{N}(t)\right),
$$

where $k=1, \ldots, N$ and $u_{0}^{N}(t):=u_{1}^{N}(t), u_{N}^{N}(t)=: u_{N+1}^{N}(t)$ (the Neumann BC). We define $h_{N}=\frac{1}{N}$.

We can set

$$
U^{N}(t):=\left[u_{1}^{N}(t), \ldots, u_{N}^{N}(t)\right]
$$

and rewrite the system (7.7) as

$$
\dot{U}^{N}(t)=\Delta^{N} U^{N}(t)
$$

Note that the matrix $\Delta^{N}$ has eigenvalues

$$
\lambda_{j}^{N}=-4 N^{2} \sin ^{2} \frac{(j-1) \pi}{2 N}
$$

and eigenvectors

$$
\overrightarrow{\mathbf{v}}_{j}=\left[\nu_{i j}\right]_{1 \leq i \leq N}^{T}, \quad \text { where } \quad \nu_{i j}= \begin{cases}N^{-\frac{1}{2}} & \text { if } \quad j=1,1 \leq i \leq N \\ \left(\frac{2}{N}\right)^{\frac{1}{2}} \cos \frac{(j-1)\left(i-\frac{1}{2}\right) \pi}{N} & \text { else. }\end{cases}
$$

If $V^{N}$ is the $N \times N$ matrix whose columns are $\overrightarrow{\mathbf{v}}_{j}, j=1, \ldots, N$, then we have

$$
U^{N}(t)=V^{N} \exp \left(D^{N} t\right)\left(V^{N}\right)^{T} U_{0}
$$

where $\exp \left(D^{N} t\right)$ is the diagonal matrix whose diagonal entries are $e^{\lambda_{j}^{N} t}, j=1, \ldots, N$.

Denote by $A_{i j}^{N}$ the $(i j)^{\text {th }}$ entry in the product $A^{N}:=V^{N} \exp \left(D^{N} t\right)\left(V^{N}\right)^{T}$. Define the function $H^{N}:[0, T] \times I \times I \rightarrow \mathbb{R}$ by

$$
H^{N}(t, x, y)=\frac{A_{i j}^{N}}{h_{N}} \quad \text { if } \quad(i-1) h_{N} \leq x<i h_{N}, \quad(j-1) h_{N} \leq y<j h_{N}
$$

for $i, j=1, \ldots, N$. Therefore, the solution $U^{N}(t)$ written as a function $u^{N}(t, x)$ (defined as 
$U_{k}^{N}(t)$ for $\left.(k-1) h_{N} \leq x<k h_{N}\right)$ is given by

$$
u^{N}(t, x)=\int_{I} H^{N}(t, x, y) u_{0}^{N}(y) d y \quad \text { for all }(t, x) \in[0, T] \times[0,1] .
$$

From the explicit formulae for the $\overrightarrow{\mathbf{v}}_{j}$ 's we compute

$$
H^{N}(t, x, y)=1+2 \sum_{k=1}^{N-1} e^{-4 N^{2} \sin ^{2} \frac{2 k \pi}{2 N}} \cos \frac{k\left(i-\frac{1}{2}\right) \pi}{N} \cos \frac{k\left(j-\frac{1}{2}\right) \pi}{N} .
$$

Going back to the discrete system $(E 2)$, we have

$$
\begin{gathered}
a^{N}(t, x)=\int_{I} H^{N}(t, x, y) a_{0}^{N}(y) d y+\int_{0}^{t} \int_{I} H^{N}(s, x, y) f_{1}^{N}(t-s, y) d y d s, \\
b^{N}(t, x)=\int_{I} H^{N}(t, x, y) b_{0}^{N}(y) d y+\int_{0}^{t} \int_{I} H^{N}(s, x, y) f_{2}^{N}(t-s, y) d y d s, \\
c^{N}(t, x)=\int_{I} H^{N}(t, x, y) c_{0}^{N}(y) d y+\int_{0}^{t} \int_{I} H^{N}(s, x, y) f_{3}^{N}(t-s, y) d y d s,
\end{gathered}
$$

where $a_{0}^{N}(y):=\int_{(k-1) h_{N}}^{k h_{N}} a_{0}(u) d u, b_{0}^{N}(y):=\int_{(k-1) h_{N}}^{k h_{N}} b_{0}(u) d u$ and $c_{0}^{N}(y):=\int_{(k-1) h_{N}}^{k h_{N}} c_{0}(u) d u$ if $(k-1) h_{N} \leq y<k h_{N}$, and $f_{1}^{N}(t, y):=-k_{1} a^{N}(t, y) b^{N}(t, y)+k_{-1} c^{N}(t, y), f_{2}^{N}(t, y):=$ $-k_{1} a^{N}(t, y) b^{N}(t, y)+k_{-1} c^{N}(t, y)$ and $f_{3}^{N}(t, y):=k_{1} a^{N}(t, y) b^{N}(t, y)-k_{-1} c^{N}(t, y)$.

\subsubsection{Convergence of $H^{N}(t, x, y)$ to $H(t, x, y)$}

Fix $t>0$. Recall that

$$
H(t, x, y)=1+2 \sum_{j=1}^{\infty} e^{-j^{2} \pi^{2} t} \cos (j \pi x) \cos (j \pi y) \quad \text { for } t \geq 0, x, y \in I
$$

and

$$
H^{N}(t, x, y)=\left\{\begin{array}{l}
1+2 \sum_{j=1}^{N-1} e^{-j^{2} \pi^{2} t\left(\frac{\sin \frac{j \pi}{2 N}}{\frac{j \pi}{2 N}}\right)^{2}} \cos \frac{j\left(k-\frac{1}{2}\right) \pi}{N} \cos \frac{j\left(i-\frac{1}{2}\right) \pi}{N} \\
\text { if } t>0, \frac{k-1}{N} \leq x<\frac{k}{N}, \frac{i-1}{N} \leq y<\frac{i}{N}, i, k=1, \ldots, N
\end{array}\right.
$$

Of course, in the expression for $H^{N}$ above, both $k$ and $i$ depend on $N$ and $x, y$ (respectively), i.e. $k=k(N, x), i=i(N, y)$.

Take an arbitrary $\varepsilon>0$ and fix an integer $m \geq 1$ such that, as the tail of a convergent 
positive term series, we have

$$
\sum_{j=m+1}^{\infty}\left(e^{-j^{2} \pi^{2} t}+e^{-4 j^{2} t}\right) \leq \frac{\varepsilon}{2}
$$

We only consider $N>m$ from now on and look at

$$
\begin{aligned}
\left|H(t, x, y)-H^{N}(t, x, y)\right| \leq & 2 \mid \sum_{j=1}^{m} e^{-j^{2} \pi^{2} t} \cos (j \pi x) \cos (j \pi y) \\
& -\sum_{j=1}^{m} e^{-j^{2} \pi^{2} t\left(\frac{\sin \frac{j \pi}{2 N}}{\frac{j \pi}{2 N}}\right)^{2}} \cos \frac{j\left(k-\frac{1}{2}\right) \pi}{N} \cos \frac{j\left(i-\frac{1}{2}\right) \pi}{N} \mid \\
& +\sum_{j=m+1}^{\infty} e^{-j^{2} \pi^{2} t}+\sum_{j=m+1}^{\infty} e^{-4 j^{2} t},
\end{aligned}
$$

where we have used $\frac{2}{\pi}<\frac{\sin \frac{j \pi}{2 N}}{\frac{j \pi}{2 N}}<1$ for all $1 \leq j \leq N$. With $m$ thus fixed, it remains to show that

$$
\left|\sum_{j=1}^{m}\left[e^{-j^{2} \pi^{2} t} \cos (j \pi x) \cos (j \pi y)-e^{-j^{2} \pi^{2} t\left(\frac{\sin \frac{j \pi}{2 N}}{\frac{j \pi}{2 N}}\right)^{2}} \cos \frac{j\left(k-\frac{1}{2}\right) \pi}{N} \cos \frac{j\left(i-\frac{1}{2}\right) \pi}{N}\right]\right| \leq \frac{\varepsilon}{4}
$$

for $N$ sufficiently large. Since $m$ is a fixed positive integer, it is sufficient to prove that for sufficiently large $N$ we have:

$$
\left|e^{-j^{2} \pi^{2} t} \cos (j \pi x) \cos (j \pi y)-e^{-j^{2} \pi^{2} t\left(\frac{\sin \frac{j \pi}{2 N}}{\frac{j \pi}{2 N}}\right)^{2}} \cos \frac{j\left(k-\frac{1}{2}\right) \pi}{N} \cos \frac{j\left(i-\frac{1}{2}\right) \pi}{N}\right| \leq \frac{\varepsilon}{4 m}
$$

for all $j=1, \ldots, m$. So, fix $j \in\{1, \ldots, m\}$. We have

$$
\lim _{N \rightarrow \infty} \frac{j \pi}{2 N}=0, \text { so } \quad \lim _{N \rightarrow \infty} e^{-j^{2} \pi^{2} t\left(\frac{\sin \frac{j \pi}{2 N}}{\frac{j \pi}{2 N}}\right)^{2}}=e^{-j^{2} \pi^{2} t} \quad \text { since } \quad \frac{\sin \frac{j \pi}{2 N}}{\frac{j \pi}{2 N}} \underset{N \rightarrow \infty}{\longrightarrow} 1 .
$$

Recall that we also have

$$
\frac{k(N, x)-1}{N} \leq x<\frac{k(N, x)}{N} \text { and } \frac{i(N, y)-1}{N} \leq y<\frac{k(N, y)}{N}
$$

(where we re-introduced the dependence of $k, i$ on $N, x, y$ to make the point that they vary 
with $N$ for $x, y$ fixed). It follows that

$$
-\frac{j \pi}{2 N} \leq j \pi x-j \pi \frac{k(N, x)-\frac{1}{2}}{N}<\frac{j \pi}{2 N} \quad \text { and } \quad-\frac{j \pi}{2 N} \leq j \pi y-j \pi \frac{i(N, y)-\frac{1}{2}}{N}<\frac{j \pi}{2 N}
$$

i.e., both

$$
\left|j \pi x-\frac{j\left(k(N, x)-\frac{1}{2}\right) \pi}{N}\right| \leq \frac{j \pi}{2 N} \quad \text { and } \quad\left|j \pi y-\frac{j\left(i(N, y)-\frac{1}{2}\right) \pi}{N}\right| \leq \frac{j \pi}{2 N}
$$

Thus,

$$
\lim _{N \rightarrow \infty} \cos \frac{j\left(k(N, x)-\frac{1}{2}\right) \pi}{N}=\cos (j \pi x) \quad \text { and } \quad \lim _{N \rightarrow \infty} \cos \frac{j\left(i(N, y)-\frac{1}{2}\right) \pi}{N}=\cos (j \pi y) .
$$

By (6.18), $(L)$ we get that for each $j \in\{1, \ldots, m\}$, there exists $N(j)$ positive integer such that (7.12) holds for all $N \geq N(j)$. Take $N \geq \max _{j=1, \ldots, m} N(j)$ to conclude

$$
\left|H^{N}(t, x, y)-H(t, x, y)\right| \leq \varepsilon \text { for all } x, y \in I .
$$

Note that $N(j)$ can be chosen independently of $x$ and/or $y$, because the $(L)$ limits above are approached uniformly with respect to $x, y$ (because of (7.14) and the fact that the cosine function is Lipschitz).

\subsubsection{A special function}

Let $f_{n}(t):=\sum_{k=1}^{n} e^{-k^{2} t}$ defined on $(0, \infty)$. Clearly, $\left\{f_{n}\right\}_{n}$ is an increasing sequence of positive decreasing functions on $(0, \infty)$.

Note that, for every $n \in \mathbb{N}$ and every $T>0$, we have

$$
\begin{aligned}
\int_{0}^{T} f_{n}(t) d t & =\left.\sum_{k=1}^{n} \frac{e^{-k^{2} t}}{-k^{2}}\right|_{0} ^{T}=\sum_{k=1}^{n} \frac{1}{k^{2}}\left(1-e^{-k^{2} T}\right) \\
& \leq \sum_{k=1}^{n} \frac{1}{k^{2}}<\sum_{k=1}^{\infty} \frac{1}{k^{2}}=\frac{\pi^{2}}{6} .
\end{aligned}
$$

Thus, $f_{n}$ is integrable on $(0, \infty)$ for all $n \geq 1$ and $\int_{0}^{\infty} f_{n}(t) d t \leq \frac{\pi^{2}}{6}$. By the Monotone Convergence Theorem, the limiting function $f(t):=\sum_{k=1}^{\infty} e^{-k^{2} t}$ (which is positive and decreasing) 
is also integrable on $(0, \infty)$ and

$$
\int_{0}^{\infty} f(t) d t \leq \frac{\pi^{2}}{6}
$$

We shall next bound $H$ and $H^{N}$ in terms of this special function $f$. From (7.8) we deduce

$$
\begin{aligned}
\left|H^{N}(t, x, y)\right| & \leq 1+2 \sum_{k=1}^{N-1} e^{-4 N^{2} \sin ^{2} \frac{2 k \pi}{2 N}} \\
& =1+2 \sum_{k=1}^{N-1} e^{-k^{2} \pi^{2} t\left(\frac{\sin \frac{2 k \pi}{2 N}}{\frac{k \pi}{2 N}}\right)^{2}}
\end{aligned}
$$

It is easy to see that $g(x):=\frac{\sin x}{x}$ is positive and decreasing on $\left[0, \frac{\pi}{2}\right]$ (at $x=0$ we define $g(0)=1$, obviously). Therefore, we have

$$
\frac{2}{\pi}=\frac{1}{\frac{\pi}{2}}<\frac{\sin \frac{k \pi}{2 N}}{\frac{k \pi}{2 N}}<1 \quad \text { for } k=1, \ldots, N-1
$$

and so

$$
\frac{4}{\pi^{2}}<\left(\frac{\sin \frac{k \pi}{2 N}}{\frac{k \pi}{2 N}}\right)^{2}<1 \quad \text { for } k=1, \ldots, N-1 .
$$

By (7.16) above, we infer

$$
\begin{aligned}
\left|H^{N}(t, x, y)\right| & \leq 1+2 \sum_{k=1}^{N-1} e^{-4 k^{2} t} \\
& <1+2 f(4 t)
\end{aligned}
$$

But in Subsection 7.1.3 we proved that for any $t>0, H^{N}(t, \cdot, \cdot)$ converges uniformly to $H(t, \cdot, \cdot)$, so we also get

$$
|H(t, x, y)| \leq 1+2 f(4 t) .
$$


In particular, for any time interval $\left[t_{1}, t_{2}\right]$ with $t_{2}>t_{1} \geq 0$, we have

$$
\begin{aligned}
\int_{t_{1}}^{t_{2}} \int_{I}|\tilde{H}(t, x, y)| d y d t & \leq\left(t_{2}-t_{1}\right)+2 \int_{t_{1}}^{t_{2}} f(4 t) d t \\
& \left.\leq\left(t_{2}-t_{1}\right)+\frac{1}{2} \int_{0}^{\infty} f(t) d t \quad \text { (change } \pi^{2} t \longleftrightarrow t\right) \\
& \leq\left(t_{2}-t_{1}\right)+\frac{1}{2} \frac{\pi^{2}}{6} \\
& =\left(t_{2}-t_{1}\right)+\frac{\pi^{2}}{12}
\end{aligned}
$$

where $\tilde{H}$ is either $H$ or $H^{N}$ (for any integer $N \geq 2$ ).

\subsubsection{Convergence of $a_{0}^{N}(x)$ to $a_{0}$ in $L^{p}(0,1)$}

Let $1 \leq p<\infty$ and $a_{0} \in L^{p}(0,1)$.

\section{Claim :}

$$
\left\|a_{0}^{N}-a_{0}\right\|_{L^{p}(0,1)} \underset{N \rightarrow \infty}{\longrightarrow} 0
$$

where $a_{0}^{N}(x)=\int_{(k-1) h}^{k h} a_{0}(y) d y$ if $(k-1) h \leq x<k h$ for $k=1, \cdots, N$ and $h=\frac{1}{N}$ proof Take $\delta>0$. Since $C_{c}^{\infty}(0,1)$ is dense in $L^{p}(0,1)$, there exists $\varphi \in C_{c}^{\infty}(0,1)$ such that

$$
\left\|\varphi-a_{0}\right\|_{L^{p}(0,1)} \leq \frac{\delta}{3}
$$

Define

$$
\varphi^{N}(x)=\int_{(k-1) h}^{k h} \varphi(y) d y \text { if }(k-1) h \leq x<k h \text { for } k=1, \cdots, N .
$$

So for sufficient large $N$ we have

$$
\left\|\varphi-\varphi^{N}\right\|_{L^{p}(0,1)}^{p}=\sum_{k=1}^{N} \int_{(k-1) h}^{k h}\left|\varphi(x)-f_{(k-1) h}^{k h} \varphi(z) d z\right|^{p} d x .
$$

Set $\varphi\left(z_{N}\right)=\int_{(k-1) h}^{k h} \varphi(z) d z$, where $\frac{k-1}{N}<z_{N}<\frac{k}{N}$, then by the mean value theorem we have

$$
\left|\varphi(x)-\varphi\left(z_{N}\right)\right| \leq\left\|\varphi^{\prime}\right\|_{\infty}\left|x-z_{N}\right|
$$


Thus

$\left\|\varphi-\varphi^{N}\right\|_{L^{p}(0,1)}^{p}=\left\|\varphi^{\prime}\right\|_{\infty}^{p} \sum_{k=1}^{N} \int_{(k-1) h}^{k h}\left|x-z_{N}\right|^{p} d x \leq\left\|\varphi^{\prime}\right\|_{\infty}^{p} \sum_{k=1}^{N} \int_{(k-1) h}^{k h} \frac{1}{N^{p}} d x=\frac{1}{N^{p}}\left\|\varphi^{\prime}\right\|_{\infty}^{p} \underset{N \rightarrow \infty}{\longrightarrow} 0$.

Thus $\left\|\varphi-\varphi^{N}\right\|_{L^{p}(0,1)} \leq \frac{\delta}{3}$ for sufficient large $N$.

Furthermore,

$$
\begin{array}{r}
\left\|\varphi^{N}-a_{0}^{N}\right\|_{L^{p}(0,1)}^{p}=\int_{I}\left|\varphi^{N}(x)-a_{0}^{N}(x)\right|^{p} d x \leq \sum_{k=1}^{N} \int_{(k-1) h}^{k h} f_{(k-1) h}^{k h}\left|\varphi(y)-a_{0}(y)\right|^{p} d y d x \\
=\sum_{k=1}^{N} \frac{1}{N} \int_{(k-1) h}^{k h}\left|\varphi(y)-a_{0}(y)\right|^{p} d y=\sum_{k=1}^{N} \frac{1}{N} N \int_{(k-1) h}^{k h}\left|\varphi(y)-a_{0}(y)\right|^{p} d y=\int_{I}\left|\varphi(y)-a_{0}(y)\right|^{p} d y,
\end{array}
$$

Thus,

$$
\left\|\varphi^{N}-a_{0}^{N}\right\|_{L^{p}(0,1)} \leq\left\|\varphi-a_{0}\right\|_{L^{p}(0,1)} \leq \frac{\delta}{3}
$$

The triangle inequality now yields

$$
\left\|a_{0}-a_{0}^{N}\right\|_{L^{p}(0,1)} \leq\left\|a_{0}-\varphi\right\|_{L^{p}(0,1)}+\left\|\varphi-\varphi^{N}\right\|_{L^{p}(0,1)}+\left\|\varphi^{N}-a_{0}^{N}\right\|_{L^{p}(0,1)} \leq \delta
$$




\section{Bibliography}

[1] David F Anderson. A proof of the global attractor conjecture in the single linkage class case. SIAM Journal on Applied Mathematics, 71(4):1487-1508, 2011.

[2] David Angeli, Patrick De Leenheer, and Eduardo D Sontag. A petri net approach to the study of persistence in chemical reaction networks. Mathematical biosciences, 210(2):598-618, 2007.

[3] Murad Banaji, Pete Donnell, and Stephen Baigent. P matrix properties, injectivity, and stability in chemical reaction systems. SIAM Journal on Applied Mathematics, 67(6):1523-1547, 2007.

[4] Murad Banaji and Casian Pantea. Some results on injectivity and multistationarity in chemical reaction networks. SIAM Journal on Applied Dynamical Systems, 15(2):807869, 2016.

[5] Elena Beretta, Michiel Bertsch, and Roberta Passo. Nonnegative solutions of a fourthorder nonlinear degenerate parabolic equation. Archive for rational mechanics and analysis, 129(2):175-200, 1995.

[6] F. Bernis et al. Finite speed of propagation and continuity of the interface for thin viscous flows. Advances in Differential Equations, 1(3):337-368, 1996.

[7] F. Bernis and A. Friedman. Higher order nonlinear degenerate parabolic equations. Journal of Differential Equations, 83(1):179-206, 1990.

[8] F. Bernis, L. Peletier, and S. Williams. Source type solutions of a fourth order nonlinear degenerate parabolic equation. Nonlinear Analysis: Theory, Methods $\&$ Applications, 18(3):217-234, 1992.

[9] A. Bertozzi and M. Pugh. The lubrication approximation for thin viscous films: the moving contact line with a'porous media'cut-off of van der waals interactions. Nonlinearity, 7(6):1535, 1994.

[10] A. Bertozzi and M. Pugh. The lubrication approximation for thin viscous films: regularity and long-time behavior of weak solutions. Communications on pure and applied mathematics, 49(2):85-123, 1996. 
[11] E. Carlen and S. Ulusoy. Asymptotic equipartition and long time behavior of solutions of a thin-film equation. Journal of Differential Equations, 241(2):279-292, 2007.

[12] EA Carlen, S Ulusoy, et al. An entropy dissipation-entropy estimate for a thin film type equation. Communications in Mathematical Sciences, 3(2):171-178, 2005.

[13] J. Carrillo and G. Toscani. Long-time asymptotics for strong solutions of the thin film equation. Communications in mathematical physics, 225(3):551-571, 2002.

[14] Wenxiong Chen, Congming Li, and Eric S Wright. On a nonlinear parabolic systemmodeling chemical reactions in rivers. COMMUNICATIONS ON PURE AND APPLIED ANALYSIS, 4(4):889, 2005.

[15] Mourad Choulli, Laurent Kayser, et al. Observations on gaussian upper bounds for neumann heat kernels. Bulletin of the Australian Mathematical Society, 92(03):429439, 2015.

[16] Carsten Conradi, Dietrich Flockerzi, Jörg Raisch, and Jörg Stelling. Subnetwork analysis reveals dynamic features of complex (bio) chemical networks. Proceedings of the National Academy of Sciences, 104(49):19175-19180, 2007.

[17] Thierry Coulhon and Alexander Grigoryan. Random walks on graphs with regular volume growth. Geometric and Functional Analysis, 8(4):656-701, 1998.

[18] Gheorghe Craciun. Toric differential inclusions and a proof of the global attractor conjecture. arXiv preprint arXiv:1501.02860, 2015.

[19] Gheorghe Craciun, Alicia Dickenstein, Anne Shiu, and Bernd Sturmfels. Toric dynamical systems. Journal of Symbolic Computation, 44(11):1551-1565, 2009.

[20] Gheorghe Craciun and Martin Feinberg. Multiple equilibria in complex chemical reaction networks: I. the injectivity property. SIAM Journal on Applied Mathematics, 65(5):1526-1546, 2005.

[21] Gheorghe Craciun and Martin Feinberg. Multiple equilibria in complex chemical reaction networks: Ii. the species-reaction graph. SIAM Journal on Applied Mathematics, 66(4):1321-1338, 2006.

[22] Gheorghe Craciun, Fedor Nazarov, and Casian Pantea. Persistence and permanence of mass-action and power-law dynamical systems. SIAM Journal on Applied Mathematics, 73(1):305-329, 2013.

[23] Laurent Desvillettes and Klemens Fellner. Exponential decay toward equilibrium via entropy methods for reaction-diffusion equations. Journal of mathematical analysis and applications, 319(1):157-176, 2006.

[24] Laurent Desvillettes, Klemens Fellner, and Bao Quoc Tang. Trend to equilibrium for reaction-diffusion systems arising from complex balanced chemical reaction networks. arXiv preprint arXiv:1604.04536, 2016. 
[25] Martin Feinberg. Complex balancing in general kinetic systems. Archive for rational mechanics and analysis, 49(3):187-194, 1972.

[26] Klemens Fellner, Wolfgang Prager, and Bao Q Tang. The entropy method for reactiondiffusion systems without detailed balance: first order chemical reaction networks. arXiv preprint arXiv:1504.08221, 2015.

[27] Alexander N Gorban, Hrachya P Sargsyan, and Hafiz A Wahab. Quasichemical models of multicomponent nonlinear diffusion. Mathematical Modelling of Natural Phenomena, 6(5):184-262, 2011.

[28] Alexander Grigoryan. Upper bounds of derivatives of the heat kernel on an arbitrary complete manifold. Journal of Functional Analysis, 127(2):363-389, 1995.

[29] Fritz Horn. Necessary and sufficient conditions for complex balancing in chemical kinetics. Archive for Rational Mechanics and Analysis, 49(3):172-186, 1972.

[30] Fritz Horn and Roy Jackson. General mass action kinetics. Archive for rational mechanics and analysis, 47(2):81-116, 1972.

[31] Josephus Hulshof. A local analysis of similarity solutions of the thin lm equation. Report W94-22, Leiden University, 1994.

[32] Congming Li and Eric S Wright. Modeling chemical reactions in rivers: A three component reaction. Discrete and Continuous Dynamical Systems, 7(2):377-384, 2001.

[33] JL Lopez, J Soler, and G Toscani. Time rescaling and asymptotic behavior of some fourth-order degenerate diffusion equations. Computers $\&$ Mathematics with Applications, 43(6):721-736, 2002.

[34] Maya Mincheva and Marc R Roussel. Graph-theoretic methods for the analysis of chemical and biochemical networks. i. multistability and oscillations in ordinary differential equation models. Journal of mathematical biology, 55(1):61-86, 2007.

[35] Maya Mincheva and David Siegel. Stability of mass action reaction-diffusion systems. Nonlinear Analysis: Theory, Methods \& Applications, 56(8):1105-1131, 2004.

[36] T. Myers. Thin films with high surface tension. Siam Review, 40(3):441-462, 1998.

[37] Franz Rothe. Global solutions of reaction-diffusion systems, volume 1072. Springer, 1984.

[38] H. Royden and P. Fitzpatrick. Real Analysis. Prentice Hall, 2010.

[39] MNO Sadiku and CN Obiozor. A simple introduction to the method of lines. International Journal of Electrical Engineering Education, 37(3):282-296, 2000.

[40] David Siegel and Debbie MacLean. Global stability of complex balanced mechanisms. Journal of Mathematical Chemistry, 27(1-2):89-110, 2000. 
[41] Eduardo D Sontag. Structure and stability of certain chemical networks and applications to the kinetic proofreading model of t-cell receptor signal transduction. IEEE transactions on automatic control, 46(7):1028-1047, 2001.

[42] A. Tudorascu. Lubrication approximation for thin viscous films: asymptotic behavior of nonnegative solutions. Communications in Partial Differential Equations, 32(7):1147$1172,2007$.

[43] S. Ulusoy. The mathematical theory of thin film evolution. 2007.

[44] Jan G Verwer and Jesús M Sanz-Serna. Convergence of method of lines approximations to partial differential equations. Computing, 33(3-4):297-313, 1984.

[45] Yuehua Yuan and T Randall Lee. Contact angle and wetting properties. In Surface science techniques, pages 3-34. Springer, 2013. 